\title{
Control the Regioselectivity of Sulfonamidyl Radical Cyclization by Vinylic Halogen Substitution
}

\author{
Hongjian Lu, Qian Chen, and Chaozhong Li*
}

Shanghai Institute of Organic Chemistry, Chinese Academy of Sciences, 354 Fenglin Road, Shanghai 200032, P. R. China

clig@mail.sioc.ac.cn
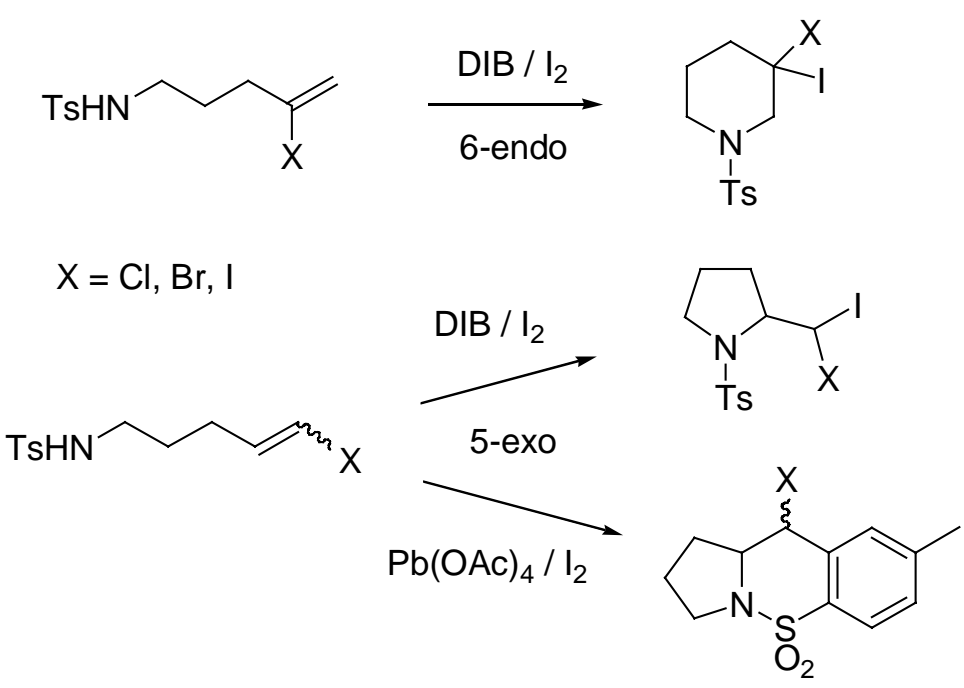

\section{Supporting Information}

1. Characterizations of substrates $1,6,9,11-13,17,19,26,29$ and $31 . \quad$ S2

2. Characterizations of products $2,3,5,7,8,10,14-16,18,23,25,27,28,30$ and $32 . \quad$ S8

$\begin{array}{ll}\text { 3. References for known compounds. } & \text { S18 }\end{array}$

4. Computational results on the cycliczation of radicals A. S19

5. ${ }^{1} \mathrm{H}$ and ${ }^{13} \mathrm{C}$ NMR spectra of compounds without elemental analyses. 


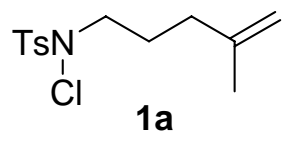

Colorless oil. ${ }^{1} \mathrm{H}$ NMR $\left(300 \mathrm{MHz}, \mathrm{CDCl}_{3}\right) \delta 1.72(3 \mathrm{H}, \mathrm{s}), 1.77-1.87(2 \mathrm{H}, \mathrm{m}), 2.09(2 \mathrm{H}, \mathrm{t}, J=7.2 \mathrm{~Hz})$, $2.47(3 \mathrm{H}, \mathrm{s}), 3.23(2 \mathrm{H}, \mathrm{t}, J=6.9 \mathrm{~Hz}), 4.73(2 \mathrm{H}, \mathrm{d}, J=14.7 \mathrm{~Hz}), 7.39(2 \mathrm{H}, \mathrm{d}, J=8.1 \mathrm{~Hz}), 7.82(2 \mathrm{H}, \mathrm{d}, J$ $=8.1 \mathrm{~Hz}) .{ }^{13} \mathrm{C}$ NMR $\left(\mathrm{CDCl}_{3}\right) \delta 21.6,22.3,25.1,34.0,56.2,110.8,129.5,129.6,130.2,144.2,145.3$. EIMS: $m / z$ (rel intensity) $287\left(\mathrm{M}^{+}, 2\right), 252$ (14), 238 (16), 198 (10), 184 (8), 155 (53), 132 (9), 91 (100). HRMS calcd for $\mathrm{C}_{13} \mathrm{H}_{18} \mathrm{NO}_{2} \mathrm{~S}\left(\mathrm{M}^{+}-\mathrm{Cl}\right)$ : 252.1058. Found: 252.1070 .

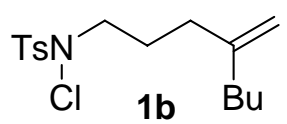

Colorless oil. ${ }^{1} \mathrm{H}$ NMR $\left(300 \mathrm{MHz}, \mathrm{CDCl}_{3}\right) \delta 0.90(3 \mathrm{H}, \mathrm{t}, J=7.2 \mathrm{~Hz}), 1.24-1.45(4 \mathrm{H}, \mathrm{m}), 1.79-1.87(2 \mathrm{H}$, m), $2.00(2 \mathrm{H}, \mathrm{t}, J=7.5 \mathrm{~Hz}), 2.09(2 \mathrm{H}, \mathrm{t}, J=7.5 \mathrm{~Hz}), 2.48(3 \mathrm{H}, \mathrm{s}), 3.23(2 \mathrm{H}, \mathrm{t}, J=6.9 \mathrm{~Hz}), 4.73(2 \mathrm{H}, \mathrm{d}$, $J=8.4 \mathrm{~Hz}), 7.39(2 \mathrm{H}, \mathrm{d}, J=8.1 \mathrm{~Hz}), 7.82(2 \mathrm{H}, \mathrm{d}, J=8.1 \mathrm{~Hz}) .{ }^{13} \mathrm{C} \mathrm{NMR}\left(\mathrm{CDCl}_{3}\right) \delta 13.9,21.7,22.4$, 25.3, 29.9, 32.3, 35.7, 56.3, 109.6, 129.5, 129.6, 130.1, 145.3, 148.4. EIMS: $m / z$ (rel intensity) 329 (M+ 6), 294 (40), 280 (17), 184 (25), 155 (72), 140 (34), 91 (100), 77 (32). HRMS calcd for $\mathrm{C}_{16} \mathrm{H}_{24} \mathrm{ClNO}_{2} \mathrm{SNa}\left(\mathrm{M}^{+}+\mathrm{Na}\right): 352.1106$. Found: 352.1109 .

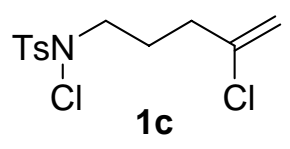

Colorless oil. ${ }^{1} \mathrm{H}$ NMR $\left(300 \mathrm{MHz}, \mathrm{CDCl}_{3}\right) \delta 1.90-1.99(2 \mathrm{H}, \mathrm{m}), 2.44-2.50(5 \mathrm{H}, \mathrm{m}), 3.25(2 \mathrm{H}, \mathrm{t}, J=6.3$ $\mathrm{Hz}), 5.22-5.24(2 \mathrm{H}, \mathrm{m}), 7.39(2 \mathrm{H}, \mathrm{d}, J=8.1 \mathrm{~Hz}), 7.81(2 \mathrm{H}, \mathrm{d}, J=8.1 \mathrm{~Hz}) .{ }^{13} \mathrm{C} \mathrm{NMR}\left(\mathrm{CDCl}_{3}\right) \delta 21.7$, 24.5, 35.5, 55.3 113.6, 129.6, 129.7, 129.9, 141.0, 145.5. EIMS: $\mathrm{m} / z$ (rel intensity) 271 ( $\mathrm{M}^{+}-\mathrm{HCl}, 3$ ), 206 (3), 184 (9), 155 (49), 139 (5), 118 (32), 91 (100), 65 (22). HRMS calcd for $\mathrm{C}_{12} \mathrm{H}_{16} \mathrm{Cl}_{2} \mathrm{NO}_{2} \mathrm{~S}$ $\left(\mathrm{M}^{+}+1\right)$ : 308.0282. Found: 308.0273 . 


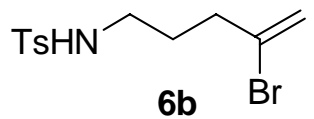

White solid. Mp: 44-46 ${ }^{\circ} \mathrm{C} .{ }^{1} \mathrm{H}$ NMR $\left(300 \mathrm{MHz}, \mathrm{CDCl}_{3}\right) \delta$ 1.69-1.78 (2H, m), 2.42-2.47 (5H, m), 2.98 $(2 \mathrm{H}, \mathrm{q}, J=6.3 \mathrm{~Hz}), 4.43(1 \mathrm{H}, \mathrm{t}, J=5.7 \mathrm{~Hz}), 5.39(1 \mathrm{H}, \mathrm{s}), 5.55(1 \mathrm{H}, \mathrm{s}), 7.32(2 \mathrm{H}, \mathrm{d}, J=7.8 \mathrm{~Hz}), 7.75$ $(2 \mathrm{H}, \mathrm{d}, J=7.8 \mathrm{~Hz}) .{ }^{13} \mathrm{C} \mathrm{NMR}\left(\mathrm{CDCl}_{3}\right) \delta 21.5,27.8,38.2,41.8,117.7,127.1,129.8,132.8,136.9$, 143.5. EIMS: $m / z$ (rel intensity) 238 (M+-Br, 13), 199 (9), 183 (11), 155 (50), 139 (14), 98 (37), 91 (100), 83 (34). Anal. calcd for $\mathrm{C}_{12} \mathrm{H}_{16} \mathrm{BrNO}_{2} \mathrm{~S}$ : C, 45.29; H, 5.07; N, 4.40. Found: C, 45.42; H, 5.20; N, 4.22 .

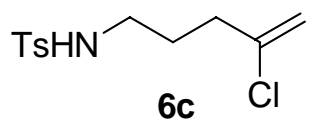

Colorless oil. ${ }^{1} \mathrm{H}$ NMR (300 MHz, $\left.\mathrm{CDCl}_{3}\right) \delta 1.68-1.78(2 \mathrm{H}, \mathrm{m}), 2.36(2 \mathrm{H}, \mathrm{t}, J=7.2 \mathrm{~Hz}), 2.43(3 \mathrm{H}, \mathrm{s})$, $2.96(2 \mathrm{H}, \mathrm{q}, J=6.6 \mathrm{~Hz}), 4.51(1 \mathrm{H}, \mathrm{t}, J=6.3 \mathrm{~Hz}), 5.13(2 \mathrm{H}, \mathrm{d}, J=9.3 \mathrm{~Hz}), 7.32(2 \mathrm{H}, \mathrm{d}, J=8.4 \mathrm{~Hz})$, $7.75(2 \mathrm{H}, \mathrm{d}, J=8.4 \mathrm{~Hz}) .{ }^{13} \mathrm{C} \mathrm{NMR}\left(\mathrm{CDCl}_{3}\right) \delta 21.5,27.1,36.0,41.9,113.2,127.1,129.8,136.8,141.2$, 143.5. EIMS: $m / z$ (rel intensity) $274\left(\mathbf{M}^{+}+1,0.4\right), 238$ (4), 184 (8), 155 (49), 139 (6), 118 (50), 91 (100), 65 (31). Anal. calcd for $\mathrm{C}_{12} \mathrm{H}_{16} \mathrm{ClNO}_{2} \mathrm{~S}$ : C, 52.64; H, 5.89; N, 5.12. Found: C, 52.55; H, 5.98; N, 4.91 .

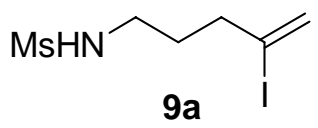

Colorless oil. ${ }^{1} \mathrm{H}$ NMR $\left(300 \mathrm{MHz}, \mathrm{CDCl}_{3}\right) \delta$ 1.75-1.86 (2H, m), 2.42-2.54 (2H, m), $2.97(3 \mathrm{H}, \mathrm{s}), 3.12-$ $3.25(2 \mathrm{H}, \mathrm{m}), 4.30(1 \mathrm{H}, \mathrm{br}), 5.76(1 \mathrm{H}, \mathrm{s}), 6.11(1 \mathrm{H}, \mathrm{s}) .{ }^{13} \mathrm{C} \mathrm{NMR}\left(\mathrm{CDCl}_{3}\right) \delta 29.3,40.3,41.6,42.0$, 110.2, 126.8. EIMS: $m / z$ (rel intensity) $290\left(\mathrm{M}^{+}+1,0.4\right), 162$ (6), 121 (3), 108 (58), 98 (18), 83 (100), 79 (12), 67 (19). Anal. calcd for $\mathrm{C}_{6} \mathrm{H}_{12} \mathrm{INO}_{2} \mathrm{~S}: \mathrm{C}, 24.92 ; \mathrm{H}, 4.18$; N, 4.84. Found: C, 24.89; H, 4.24; N, 4.94 .

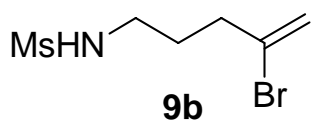


Colorless oil. ${ }^{1} \mathrm{H}$ NMR (300 MHz, $\left.\mathrm{CDCl}_{3}\right) \delta 1.81-1.90(2 \mathrm{H}, \mathrm{m}), 2.53(2 \mathrm{H}, \mathrm{t}, J=7.2 \mathrm{~Hz}), 2.98(3 \mathrm{H}, \mathrm{s})$, 3.13-3.21 (2H, m), $4.34(1 \mathrm{H}, \mathrm{br}), 5.46(1 \mathrm{H}, \mathrm{s}), 5.65(1 \mathrm{H}, \mathrm{s}) .{ }^{13} \mathrm{C} \mathrm{NMR}\left(\mathrm{CDCl}_{3}\right) \delta 28.2,38.1,40.2,41.8$, 117.9, 132.7. EIMS: $m / z$ (rel intensity) $244\left(\mathrm{M}^{+}\left({ }^{81} \mathrm{Br}\right)+1,1\right), 242\left(\mathrm{M}^{+}\left({ }^{79} \mathrm{Br}\right)+1,1\right), 162(13), 149(4), 121$ (7), 108 (64), 98 (28), 83 (100). Anal. calcd for $\mathrm{C}_{6} \mathrm{H}_{12} \mathrm{BrNO}_{2} \mathrm{~S}$ : C, 29.76; H, 5.00; N, 5.78. Found: C, $29.65 ; \mathrm{H}, 5.09 ; \mathrm{N}, 5.74$.

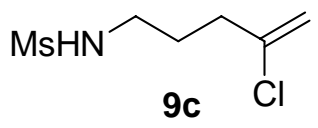

Colorless oil. ${ }^{1} \mathrm{H}$ NMR (300 MHz, $\left.\mathrm{CDCl}_{3}\right) \delta 1.86(2 \mathrm{H}, \mathrm{tt}, J=6.6,6.9 \mathrm{~Hz}), 2.45(2 \mathrm{H}, \mathrm{t}, J=7.2 \mathrm{~Hz}), 2.97$ $(3 \mathrm{H}, \mathrm{s}), 3.17(2 \mathrm{H}, \mathrm{q}, J=6.9 \mathrm{~Hz}), 4.40(\mathrm{lH}, \mathrm{br}), 5.21(2 \mathrm{H}, \mathrm{s}) .{ }^{13} \mathrm{C} \mathrm{NMR}\left(\mathrm{CDCl}_{3}\right) \delta 27.6,36.0,40.4,42.0$, 113.3, 141.3. EIMS: m/z (rel intensity) $198\left(\mathrm{M}^{+}+1,1\right), 162$ (3), 121 (10), 118 (25), 108 (100), 102 (6), 79 (18), 67 (15). Anal. calcd for $\mathrm{C}_{6} \mathrm{H}_{12} \mathrm{ClNO}_{2} \mathrm{~S}: \mathrm{C}, 36.45 ; \mathrm{H}, 6.12 ; \mathrm{N}, 7.09$. Found: C, 36.32; H, 6.22; $\mathrm{N}, 6.91$.

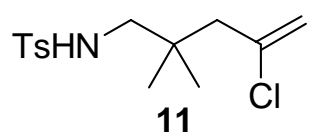

White solid. Mp: 80-82 ${ }^{\circ} \mathrm{C} .{ }^{1} \mathrm{H}$ NMR (300 MHz, $\left.\mathrm{CDCl}_{3}\right) \delta 0.96(6 \mathrm{H}, \mathrm{s}), 2.30(2 \mathrm{H}, \mathrm{s}), 2.43(3 \mathrm{H}, \mathrm{s}), 2.76$ $(2 \mathrm{H}, \mathrm{d}, J=7.2 \mathrm{~Hz}), 4.84(1 \mathrm{H}, \mathrm{br}), 5.10(1 \mathrm{H}, \mathrm{s}), 5.25(1 \mathrm{H}, \mathrm{s}), 7.31(2 \mathrm{H}, \mathrm{d}, J=8.4 \mathrm{~Hz}), 7.74(2 \mathrm{H}, \mathrm{d}, J=$ $8.4 \mathrm{~Hz}) .{ }^{13} \mathrm{C} \mathrm{NMR}\left(\mathrm{CDCl}_{3}\right) \delta 21.5,25.3,34.7,48.0,52.6,116.4,127.0,129.7,137.1,138.7,143.4$. EIMS: $m / z$ (rel intensity) $301\left(\mathrm{M}^{+}, 0.4\right), 184$ (33), 155 (68), 146 (21), 139 (5), 91 (100), 77 (9), 65 (28). Anal. calcd for $\mathrm{C}_{14} \mathrm{H}_{20} \mathrm{ClNO}_{2} \mathrm{~S}$ : C, 55.71; H, 6.68; N, 4.64. Found: C, 55.72; H, 6.67; N, 4.64.

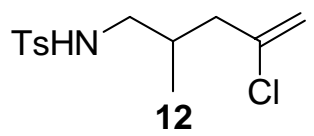

Colorless oil. ${ }^{1} \mathrm{H}$ NMR $\left(300 \mathrm{MHz}, \mathrm{CDCl}_{3}\right) \delta 0.89(3 \mathrm{H}, \mathrm{d}, J=6.6 \mathrm{~Hz}), 1.93-2.18(2 \mathrm{H}, \mathrm{m}), 2.35-2.40$ $(1 \mathrm{H}, \mathrm{m}), 2.42(3 \mathrm{H}, \mathrm{s}), 2.76-2.93(2 \mathrm{H}, \mathrm{m}), 5.02(1 \mathrm{H}, \mathrm{br}), 5.16(1 \mathrm{H}, \mathrm{s}), 5.30(1 \mathrm{H}, \mathrm{s}), 7.31(2 \mathrm{H}, \mathrm{d}, J=8.4$ $\mathrm{Hz}), 7.75(2 \mathrm{H}, \mathrm{d}, J=8.4 \mathrm{~Hz}) .{ }^{13} \mathrm{C} \mathrm{NMR}\left(\mathrm{CDCl}_{3}\right) \delta 16.8,21.4,31.1,43.6,48.1,114.1,127.1,129.7$, 137.1, 140.5, 143.4. EIMS: $m / z$ (rel intensity) $288\left(\mathrm{M}^{+}+1,0.3\right), 252$ (3), 184 (26), 155 (75), 132 (54), 
112 (6), 91 (100), 65 (31). Anal. calcd for $\mathrm{C}_{13} \mathrm{H}_{18} \mathrm{ClNO}_{2} \mathrm{~S}$ : C, 54.25; H, 6.30; N, 4.87. Found: C, 54.37;

H, 6.30; N, 4.53.

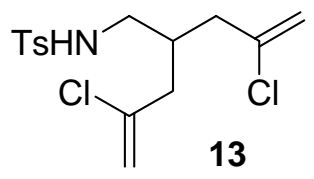

White solid. ${ }^{1} \mathrm{H}$ NMR (300 MHz, $\left.\mathrm{CDCl}_{3}\right) \delta$ 2.25-2.36 (4H, m), $2.43(3 \mathrm{H}, \mathrm{s}), 2.94-2.99(2 \mathrm{H}, \mathrm{dd}, J=5.2$, $6.6 \mathrm{~Hz}), 4.45(2 \mathrm{H}, \mathrm{t}, J=6.6 \mathrm{~Hz}), 5.20(4 \mathrm{H}, \mathrm{t}, J=12.6 \mathrm{~Hz}), 7.31(2 \mathrm{H}, \mathrm{d}, J=8.4 \mathrm{~Hz}), 7.73(2 \mathrm{H}, \mathrm{d}, J=$ $8.4 \mathrm{~Hz}) .{ }^{13} \mathrm{C} \mathrm{NMR}\left(\mathrm{CDCl}_{3}\right) \delta 21.5,33.8,40.7,44.6,114.9,127.1,129.8,136.8,139.9,143.6$. EIMS: m/z (rel intensity) $348\left(\mathrm{M}^{+}+1,0.1\right), 312$ (3), 192 (23), 184 (22), 155 (74), 116 (23), 91 (100), 65 (40). HRMS calcd for $\mathrm{C}_{15} \mathrm{H}_{19} \mathrm{Cl}_{2} \mathrm{NO}_{2} \mathrm{SNa}\left(\mathrm{M}^{+}+\mathrm{Na}\right): 370.0408$. Found: 370.0406 .

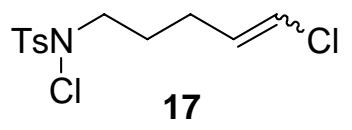

Colorless oil. Z:E = 67:33. ${ }^{1} \mathrm{H}$ NMR $\left(300 \mathrm{MHz}, \mathrm{CDCl}_{3}\right) \delta$ 1.67-1.80 (2H, m), 2.11/2.25 (2H, 2q, $J=7.2$ $\mathrm{Hz}), 2.41(3 \mathrm{H}, \mathrm{s}), 3.17(2 \mathrm{H}, \mathrm{q}, J=6.3 \mathrm{~Hz}), 5.68-6.03(2 \mathrm{H}, \mathrm{m}), 7.33(2 \mathrm{H}, \mathrm{d}, J=8.1 \mathrm{~Hz}), 7.75(2 \mathrm{H}, \mathrm{d}, J$ $=8.1 \mathrm{~Hz}) .{ }^{13} \mathrm{C} \mathrm{NMR}\left(\mathrm{CDCl}_{3}\right) \delta 21.8,23.6 / 26.2,25.9 / 27.2,56.0 / 55.7,119.5 / 118.4,129.6,129.7,129.8$, 130.0/132.0, 145.5. EIMS: $m / z$ (rel intensity) $274\left(\mathrm{M}^{+}-\mathrm{Cl}, 0.3\right), 272$ (0.8), 236 (3), 224 (18), 184 (4), 155 (33), 91 (100), 65 (25). HRMS calcd for $\mathrm{C}_{12} \mathrm{H}_{15} \mathrm{C}_{12} \mathrm{NO}_{2} \mathrm{~S}\left(\mathrm{M}^{+}-\mathrm{Cl}\right)$ : 272.0510. Found: 272.0512.

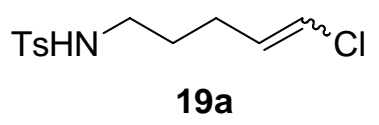

Colorless oil. $\mathrm{Z}: \mathrm{E}=67: 33 .{ }^{1} \mathrm{H}$ NMR $\left(300 \mathrm{MHz}, \mathrm{CDCl}_{3}\right) \delta 1.54-1.65(2 \mathrm{H}, \mathrm{m}), 2.07 / 2.21(2 \mathrm{H}, 2 \mathrm{q}, J=7.2$ Hz), $2.44(3 \mathrm{H}, \mathrm{s}), 2.95(2 \mathrm{H}, \mathrm{q}, J=6.6 \mathrm{~Hz}), 4.40-4.46(1 \mathrm{H}, \mathrm{m}), 5.64-6.05(2 \mathrm{H}, \mathrm{m}), 7.32(2 \mathrm{H}, \mathrm{d}, J=8.1$ $\mathrm{Hz}), 7.75(2 \mathrm{H}, \mathrm{d}, J=8.1 \mathrm{~Hz}) .{ }^{13} \mathrm{C} \mathrm{NMR}\left(\mathrm{CDCl}_{3}\right) \delta 21.5,24.0 / 27.7,28.3 / 28.7,42.5 / 42.2,119.2 / 118.0$, 127.1, 129.7, 130.1/132,2, 137.0, 143.4/143.5. EIMS: $m / z$ (rel intensity) $274\left(\mathrm{M}^{+}+1,1\right), 238$ (11), 210 (3), 184 (10), 155 (66), 118 (25), 91 (100), 65 (38). Anal. calcd for $\mathrm{C}_{12} \mathrm{H}_{16} \mathrm{ClNO}_{2} \mathrm{~S}$ : C, 52.64; H, 5.89; N, 5.12. Found: C, 52.53; H, 5.93; N, 5.08. 


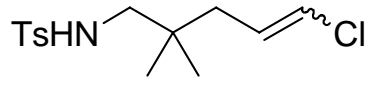

$19 b$

White solid. Z:E = 50:50. ${ }^{1} \mathrm{H}$ NMR $\left(300 \mathrm{MHz}, \mathrm{CDCl}_{3}\right) \delta 0.85(3 \mathrm{H}, \mathrm{s}), 0.91(3 \mathrm{H}, \mathrm{s}), 1.96(1 \mathrm{H}, \mathrm{d}, J=7.8$ Hz), $2.15(1 \mathrm{H}, \mathrm{dd}, J=1.5,7.8 \mathrm{~Hz}), 2.43(3 \mathrm{H}, \mathrm{s}), 2.68(2 \mathrm{H}, \mathrm{dd}, J=6.0,6.9 \mathrm{~Hz}), 4.37-4.63(1 \mathrm{H}, \mathrm{m})$, 5.68-6.11 (2H, m), $7.31(1 \mathrm{H}, \mathrm{d}, J=8.1 \mathrm{~Hz}), 7.32(1 \mathrm{H}, \mathrm{d}, J=8.1 \mathrm{~Hz}), 7.73(1 \mathrm{H}, \mathrm{d}, J=8.1 \mathrm{~Hz}), 7.74$ $(1 \mathrm{H}, \mathrm{d}, J=8.1 \mathrm{~Hz}) .{ }^{13} \mathrm{C} \mathrm{NMR}\left(\mathrm{CDCl}_{3}\right) \delta 21.4,24.7 / 24.8,34.3 / 34.8,36.5 / 40.3,52.6 / 52.7,119.2 / 120.1$, 127.0, 127.4/129.4, 129.7, 137.0/137.1, 143.3/143.4. EIMS: $m / z$ (rel intensity) $266\left(\mathrm{M}^{+}-\mathrm{Cl}, 6\right), 226(2)$, 184 (35), 172(3), 155 (93), 126 (11), 91 (100), 65 (32). Anal. calcd for $\mathrm{C}_{14} \mathrm{H}_{20} \mathrm{ClNO}_{2} \mathrm{~S}: \mathrm{C}, 55.71 ; \mathrm{H}$, $6.68 ; \mathrm{N}, 4.64$. Found: C, 55.83; H, 6.74; N, 4.61 .

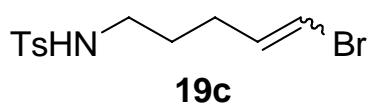

Colorless oil. Z:E = 75:25. ${ }^{1} \mathrm{H}$ NMR $\left(300 \mathrm{MHz} \mathrm{CDCl}_{3}\right) \delta 1.54-1.66(2 \mathrm{H}, \mathrm{m}), 2.05 / 2.20(2 \mathrm{H}, 2 \mathrm{q}, J=7.2$ $\mathrm{Hz}), 2.43(3 \mathrm{H}, \mathrm{s}), 2.96(2 \mathrm{H}, \mathrm{q}, J=6.9 \mathrm{~Hz}), 4.49(1 \mathrm{H}, \mathrm{br}), 5.98-6.18(2 \mathrm{H}, \mathrm{m}), 7.31(2 \mathrm{H}, \mathrm{d}, J=8.1 \mathrm{~Hz})$, $7.75(2 \mathrm{H}, \mathrm{d}, J=8.1 \mathrm{~Hz}) .{ }^{13} \mathrm{C} \mathrm{NMR}\left(\mathrm{CDCl}_{3}\right) \delta 21.5,26.7 / 28.4,28.1 / 29.7,42.5 / 42.2,108.9 / 105.4,127.1$, 129.7, 133.3/136.4, 137.0, 143.5/143.4. EIMS: $m / z$ (rel intensity) 238 ( $\mathrm{M}^{+}$-Br, 9), 210 (4), 184 (12), 155 (56), 98 (36), 91 (100), 83 (26), 65 (31). Anal. calcd for $\mathrm{C}_{12} \mathrm{H}_{16} \mathrm{BrNO}_{2} \mathrm{~S}: \mathrm{C}, 45.29 ; \mathrm{H}, 5.07 ; \mathrm{N}, 4.40$. Found: C, 45.50; H, 5.29; N, 4.36 .

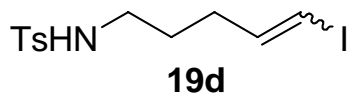

Colorless oil. Z:E = 73:27. ${ }^{1} \mathrm{H}$ NMR $\left(300 \mathrm{MHz} \mathrm{CDCl}_{3}\right) \delta$ 1.54-1.67 (2H, m), 2.01-2.19 (2H, m), 2.44 $(3 \mathrm{H}, \mathrm{s}), 2.91-3.02(2 \mathrm{H}, \mathrm{m}), 4.43(1 \mathrm{H}, \mathrm{t}, J=6.6 \mathrm{~Hz}), 5.94-6.44(2 \mathrm{H}, \mathrm{m}), 7.31(2 \mathrm{H}, \mathrm{d}, J=8.1 \mathrm{~Hz}), 7.75$ $(2 \mathrm{H}, \mathrm{d}, J=8.1 \mathrm{~Hz}) .{ }^{13} \mathrm{C} \mathrm{NMR}\left(\mathrm{CDCl}_{3}\right) \delta 21.5,27.9 / 28.2,31.8 / 32.7,42.5 / 42.2,83.6 / 75.7,127.1,129.7$, 137.0, 139.8, 143.4/144.8. EIMS: $m / z$ (rel intensity) $366\left(\mathrm{M}^{+}+1,0.8\right), 238$ (6), 194 (6), 184 (16), 155 (42), 98 (47), 91 (100), 83 (47). Anal. calcd for $\mathrm{C}_{12} \mathrm{H}_{16} \mathrm{INO}_{2} \mathrm{~S}: \mathrm{C}, 39.46 ; \mathrm{H}, 4.42$; N, 3.84. Found: C,39.66; H, 4.69; N, 3.70. 


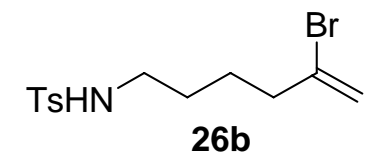

Colorless oil. ${ }^{1} \mathrm{H}$ NMR (300 MHz, $\left.\mathrm{CDCl}_{3}\right) \delta 1.41-1.60(4 \mathrm{H}, \mathrm{m}), 2.37(2 \mathrm{H}, \mathrm{t}, J=6.6 \mathrm{~Hz}), 2.43(3 \mathrm{H}, \mathrm{s})$, $2.96(2 \mathrm{H}, \mathrm{q}, J=6.6 \mathrm{~Hz}), 4.32(1 \mathrm{H}, \mathrm{t}, J=6.0 \mathrm{~Hz}), 5.37(1 \mathrm{H}, \mathrm{d}, J=1.5 \mathrm{~Hz}), 5.37(1 \mathrm{H}, \mathrm{d}, J=0.9 \mathrm{~Hz})$, $7.32(2 \mathrm{H}, \mathrm{d}, J=8.1 \mathrm{~Hz}), 7.75(2 \mathrm{H}, \mathrm{d}, J=8.1 \mathrm{~Hz}) .{ }^{13} \mathrm{C} \mathrm{NMR}\left(\mathrm{CDCl}_{3}\right) \delta 21.5,24.8,28.3,40.6,42.8$, 116.9, 127.1, 129.7, 133.8, 137.1, 143.4. EIMS: $m / z$ (rel intensity) $252\left(\mathrm{M}^{+}-\mathrm{Br}, 11\right), 210$ (5), 184 (14), 155 (57), 112 (18), 97 (29), 91 (100), 65 (29). Anal. calcd for $\mathrm{C}_{13} \mathrm{H}_{18} \mathrm{BrNO}_{2} \mathrm{~S}$ : C, 46.99; H, 5.46; N, 4.22. Found: C, 47.38; H, 5.57; N, 3.84.

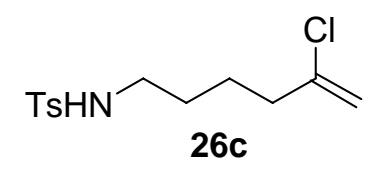

Colorless oil. ${ }^{1} \mathrm{H}$ NMR (300 MHz, $\left.\mathrm{CDCl}_{3}\right) \delta 1.45-1.59(4 \mathrm{H}, \mathrm{m}), 2.89(2 \mathrm{H}, \mathrm{t}, J=6.3 \mathrm{~Hz}), 2.43(3 \mathrm{H}, \mathrm{s})$, 2.92-3.00 (2H, m), 4.35 (1H, br), $5.10(2 \mathrm{H}, \mathrm{d}, J=14.4 \mathrm{~Hz}), 7.32(2 \mathrm{H}, \mathrm{d}, J=8.4 \mathrm{~Hz}), 7.75(2 \mathrm{H}, \mathrm{d}, J=$ $8.4 \mathrm{~Hz}) .{ }^{13} \mathrm{C} \mathrm{NMR}\left(\mathrm{CDCl}_{3}\right) \delta 21.5,24.0,28.4,38.4,42.8,112.4,127.1,129.7,137.0,142.1,143.4$. EIMS: $m / z$ (rel intensity) $288\left(\mathrm{M}^{+}+1,0.2\right), 252$ (3), 210 (8), 184 (10), 155 (67), 132 (27), 91 (100), 65 (32). Anal. calcd for $\mathrm{C}_{13} \mathrm{H}_{18} \mathrm{ClNO}_{2} \mathrm{~S}$ : C, 54.25; H, 6.30; N, 4.87. Found: C, 54.18; H, 6.18; N, 4.57.

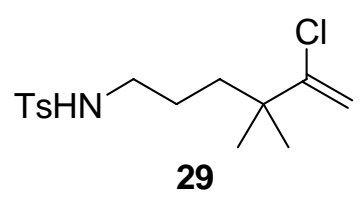

Colorless oil. ${ }^{1} \mathrm{H}$ NMR $\left(300 \mathrm{MHz}, \mathrm{CDCl}_{3}\right) \delta 1.08(6 \mathrm{H}, \mathrm{s}), 1.25-1.42(4 \mathrm{H}, \mathrm{m}), 2.43(3 \mathrm{H}, \mathrm{s}), 2.92(2 \mathrm{H}, \mathrm{q}$, $J=6.0 \mathrm{~Hz}), 4.60(1 \mathrm{H}, \mathrm{t}, J=6.0 \mathrm{~Hz}), 5.07(1 \mathrm{H}, \mathrm{t}, J=1.5 \mathrm{~Hz}), 5.15(1 \mathrm{H}, \mathrm{s}), 7.30(2 \mathrm{H}, \mathrm{d}, J=7.8 \mathrm{~Hz})$, $7.75(2 \mathrm{H}, \mathrm{d}, J=7.8 \mathrm{~Hz}) .{ }^{13} \mathrm{C} \mathrm{NMR}\left(\mathrm{CDCl}_{3}\right) \delta 21.5,24.8,26.7,37.1,41.4,43.6,111.5,127.1,129.7$, 137.0, 143.4, 150.7. EIMS: $m / z$ (rel intensity) $316\left(\mathrm{M}^{+}+1,0.9\right), 280$ (2), 210 (40), 184 (19), 160 (20), 155 (75), 91 (100), 65 (28). Anal. calcd for $\mathrm{C}_{15} \mathrm{H}_{22} \mathrm{ClNO}_{2} \mathrm{~S}$ : C, 57.04; H, 7.02; N, 4.43. Found: C, $57.05 ; \mathrm{H}, 7.01 ; \mathrm{N}, 4.30$. 


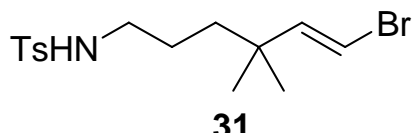

31

Colorless oil. ${ }^{1} \mathrm{H}$ NMR $\left(300 \mathrm{MHz}, \mathrm{CDCl}_{3}\right) \delta 1.23(6 \mathrm{H}, \mathrm{s}), 1.38-1.52(4 \mathrm{H}, \mathrm{m}), 2.43(3 \mathrm{H}, \mathrm{s}), 2.94(2 \mathrm{H}, \mathrm{q}$, $J=6.0 \mathrm{~Hz}), 4.34(1 \mathrm{H}, \mathrm{t}, J=6.0 \mathrm{~Hz}), 5.95-6.05(2 \mathrm{H}, \mathrm{m}), 7.31(2 \mathrm{H}, \mathrm{d}, J=8.4 \mathrm{~Hz}), 7.75(2 \mathrm{H}, \mathrm{d}, J=8.4$ $\mathrm{Hz}) .{ }^{13} \mathrm{C} \mathrm{NMR}\left(\mathrm{CDCl}_{3}\right) \delta 21.5,25.1,27.6,36.8,38.7,43.8,104.6,127.1,129.7,137.1,141.5,143.3$. ESIMS: $m / z 360.0\left[\mathrm{M}^{+}\left({ }^{79} \mathrm{Br}\right)+1\right]$. HRMS calcd for $\mathrm{C}_{15} \mathrm{H}_{22} \mathrm{BrNO}_{2} \mathrm{SNa}\left(\mathrm{M}^{+}\left({ }^{79} \mathrm{Br}\right)+\mathrm{Na}\right): 382.0451$. Found: 382.0447.

Characterization of products

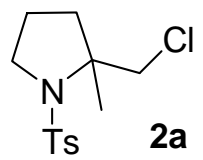

Colorless oil. ${ }^{1} \mathrm{H}$ NMR $\left(300 \mathrm{MHz}, \mathrm{CDCl}_{3}\right) \delta 1.52(3 \mathrm{H}, \mathrm{s}), 1.63-1.91(3 \mathrm{H}, \mathrm{m}), 2.25-2.33(1 \mathrm{H}, \mathrm{m}), 2.43$ $(3 \mathrm{H}, \mathrm{s}), 3.30-3.46(2 \mathrm{H}, \mathrm{m}), 3.85-3.89(2 \mathrm{H}, \mathrm{m}), 7.29(2 \mathrm{H}, \mathrm{d}, J=8.1 \mathrm{~Hz}), 7.75(2 \mathrm{H}, \mathrm{d}, J=8.1 \mathrm{~Hz}) .{ }^{13} \mathrm{C}$ NMR $\left(\mathrm{CDCl}_{3}\right) \delta 21.5,22.5,23.8,38.3,49.8,51.0,67.9,127.3,129.5,137.9,143.1$. EIMS: $\mathrm{m} / z(\mathrm{rel}$ intensity) $287\left(\mathrm{M}^{+}, 1\right), 238$ (95), 198 (3.84), 155 (44), 149 (2), 132 (3), 91 (100), 65 (23). Anal. calcd for $\mathrm{C}_{13} \mathrm{H}_{18} \mathrm{ClNO}_{2} \mathrm{~S}: \mathrm{C}, 54.25 ; \mathrm{H}, 6.30 ; \mathrm{N}, 4.87$. Found: C, 54.35; H, 6.42; N, 4.67.

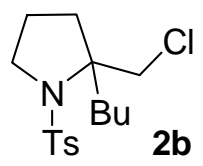

Colorless oil. ${ }^{1} \mathrm{H}$ NMR $\left(300 \mathrm{MHz}, \mathrm{CDCl}_{3}\right) \delta 0.78(3 \mathrm{H}, \mathrm{t}, J=6.6 \mathrm{~Hz}), 1.10-1.25(4 \mathrm{H}, \mathrm{m}), 1.61-1.96(5 \mathrm{H}$, m), 2.05-2.15 (1H, m), $2.34(3 \mathrm{H}, \mathrm{s}), 3.24-3.36(2 \mathrm{H}, \mathrm{m}), 3.68(1 \mathrm{H}, \mathrm{d}, J=10.8 \mathrm{~Hz}), 3.98(1 \mathrm{H}, \mathrm{d}, J=10.8$ $\mathrm{Hz}), 7.20(2 \mathrm{H}, \mathrm{d}, J=8.1 \mathrm{~Hz}), 7.68(2 \mathrm{H}, \mathrm{d}, J=8.4 \mathrm{~Hz}) .{ }^{13} \mathrm{C} \mathrm{NMR}\left(\mathrm{CDCl}_{3}\right) \delta 13.9,21.4,22.9,23.0,26.7$, 35.1, 37.3, 50.1, 51.6, 71.5, 127.2, 129.3, 138.1, 142.9. EIMS: $\mathrm{m} / z$ (rel intensity) 329 (M+ 0.2), 294 (2), 280 (78), 272 (28), 250 (3), 155 (67), 91 (100), 65 (27). Anal. calcd for $\mathrm{C}_{16} \mathrm{H}_{24} \mathrm{ClNO}_{2} \mathrm{~S}: \mathrm{C}, 58.25$; H, 7.33; N, 4.25. Found: C, 57.90; H, 7.52; N, 4.08. 


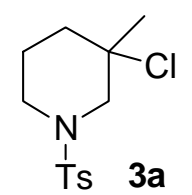

White solid. Mp: 88-90 ${ }^{\circ} \mathrm{C} .{ }^{1} \mathrm{H}$ NMR $\left(300 \mathrm{MHz}, \mathrm{CDCl}_{3}\right) \delta 1.65(3 \mathrm{H}, \mathrm{s}), 1.62-1.97(4 \mathrm{H}, \mathrm{m}), 2.44(3 \mathrm{H}$, s), 2.94-3.06 (2H, m), 3.04(1H, d, $J=11.4 \mathrm{~Hz}), 3.18(1 \mathrm{H}, \mathrm{d}, J=11.4 \mathrm{~Hz}), 7.33(2 \mathrm{H}, \mathrm{d}, J=7.8 \mathrm{~Hz})$, $7.65(2 \mathrm{H}, \mathrm{d}, J=7.8 \mathrm{~Hz}) .{ }^{13} \mathrm{C} \mathrm{NMR}\left(\mathrm{CDCl}_{3}\right) \delta 21.5,22.4,38.2,39.5,45.8,57.8,65.7,127.6,129.7$, 133.8, 143.6. EIMS: $m / z$ (rel intensity) $287\left(\mathrm{M}^{+}, 24\right), 252$ (19), 236 (8), 198 (100), 155 (67), 132 (38), 103 (18), 91 (67). Anal. calcd for $\mathrm{C}_{13} \mathrm{H}_{18} \mathrm{ClNO}_{2} \mathrm{~S}: \mathrm{C}, 54.25 ; \mathrm{H}, 6.30 ; \mathrm{N}, 4.87$. Found: C, 54.30; H, 6.45; N, 4.79.

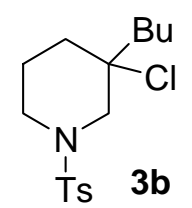

White solid. Mp: $94-96{ }^{\circ} \mathrm{C} .{ }^{1} \mathrm{H}$ NMR $\left(300 \mathrm{MHz}, \mathrm{CDCl}_{3}\right) \delta 0.90(3 \mathrm{H}, \mathrm{t}, J=7.2 \mathrm{~Hz}), 1.25-1.61$ (4H, m), 1.68-1.93 (6H, m), $2.43(3 \mathrm{H}, \mathrm{s}), 2.93-3.06(2 \mathrm{H}, \mathrm{m}), 3.13(2 \mathrm{H}, \mathrm{s}), 7.32(2 \mathrm{H}, \mathrm{d}, J=8.1 \mathrm{~Hz}), 7.65(2 \mathrm{H}, \mathrm{d}$, $J=8.1 \mathrm{~Hz}) .{ }^{13} \mathrm{C} \mathrm{NMR}\left(\mathrm{CDCl}_{3}\right) \delta 13.9,21.5,22.2,22.7,25.6,37.7,39.6,46.0,56.5,69.8,127.6,129.7$, 133.9, 143.6. EIMS: $m / z$ (rel intensity) $329\left(\mathrm{M}^{+}, 2\right), 294$ (55), 250 (32), 198 (78), 184 (16), 174 (22), 155 (90), 91 (100). Anal. calcd for $\mathrm{C}_{16} \mathrm{H}_{24} \mathrm{ClNO}_{2} \mathrm{~S}$ : C, 58.25; H, 7.33; N, 4.25. Found: C, 58.24; H, $7.34 ; \mathrm{N}, 4.07$.

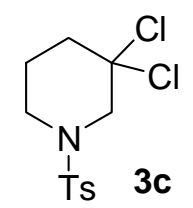

White solid. Mp: $92-94{ }^{\circ} \mathrm{C} .{ }^{1} \mathrm{H}$ NMR $\left(300 \mathrm{MHz}, \mathrm{CDCl}_{3}\right) \delta 1.88-1.95(2 \mathrm{H}, \mathrm{m}), 2.26(2 \mathrm{H}, \mathrm{t}, J=5.7 \mathrm{~Hz})$, $2.44(3 \mathrm{H}, \mathrm{s}), 3.08(2 \mathrm{H}, \mathrm{t}, J=5.4 \mathrm{~Hz}), 3.53(2 \mathrm{H}, \mathrm{s}), 7.34(2 \mathrm{H}, \mathrm{d}, J=8.4 \mathrm{~Hz}), 7.66(2 \mathrm{H}, \mathrm{d}, J=8.4 \mathrm{~Hz})$. ${ }^{13} \mathrm{C} \mathrm{NMR}\left(\mathrm{CDCl}_{3}\right) \delta 21.5,23.0,43.8,45.1,59.9,84.8,127.5,129.8,134.1,143.9$. EIMS: $\mathrm{m} / z$ (rel intensity) $307\left(\mathrm{M}^{+}, 14\right), 271$ (5), 236 (2), 198 (61), 155 (63), 116 (17), 91 (100), 65 (40). Anal. calcd for $\mathrm{C}_{12} \mathrm{H}_{15} \mathrm{Cl}_{2} \mathrm{NO}_{2} \mathrm{~S}$ : C, 46.76; H, 4.91; N, 4.54. Found: C, 47.04; H, 4.60; N, 4.17. 


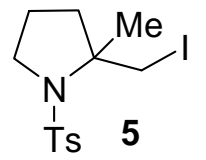

White solid. Mp: 60-62 ${ }^{\circ} \mathrm{C} .{ }^{1} \mathrm{H}$ NMR $\left(300 \mathrm{MHz}, \mathrm{CDCl}_{3}\right) \delta 1.63(3 \mathrm{H}, \mathrm{s}), 1.73-1.87(3 \mathrm{H}, \mathrm{m}), 2.20-2.25$ $(1 \mathrm{H}, \mathrm{m}), 2.42(3 \mathrm{H}, \mathrm{s}), 3.33-3.45(2 \mathrm{H}, \mathrm{m}), 3.67(1 \mathrm{H}, \mathrm{d}, J=9.9 \mathrm{~Hz}), 3.79(1 \mathrm{H}, \mathrm{d}, J=9.9 \mathrm{~Hz}), 7.29(2 \mathrm{H}, \mathrm{d}$, $J=8.1 \mathrm{~Hz}), 7.76(2 \mathrm{H}, \mathrm{d}, J=8.1 \mathrm{~Hz}) .{ }^{13} \mathrm{C} \mathrm{NMR}\left(\mathrm{CDCl}_{3}\right) \delta 17.7,21.5,22.4,24.6,41.5,49.9,66.7$, 127.4, 129.5, 137.9, 143.1. EIMS: $m / z$ (rel intensity) $364\left(\mathrm{M}^{+}-\mathrm{Me}, 4\right), 252$ (6), 238 (100), 155 (40), 139 (2), 96 (6), 91 (72), 65 (23). Anal. calcd for $\mathrm{C}_{13} \mathrm{H}_{18} \mathrm{INO}_{2} \mathrm{~S}$ : C, 41.17; H, 4.78; N, 3.69. Found: C, 41.21; $\mathrm{H}, 4.79 ; \mathrm{N}, 3.58$.

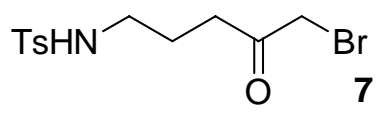

Colorless oil. ${ }^{1} \mathrm{H}$ NMR $\left(300 \mathrm{MHz}, \mathrm{CDCl}_{3}\right) \delta 1.76-1.85(2 \mathrm{H}, \mathrm{m}), 2.43(3 \mathrm{H}, \mathrm{s}), 2.76(2 \mathrm{H}, \mathrm{t}, J=6.6 \mathrm{~Hz})$, $2.97(2 \mathrm{H}, \mathrm{q}, J=6.3 \mathrm{~Hz}), 3.90(2 \mathrm{H}, \mathrm{s}), 4.48(1 \mathrm{H}, \mathrm{t}, J=6.0 \mathrm{~Hz}), 7.31(2 \mathrm{H}, \mathrm{d}, J=8.1 \mathrm{~Hz}), 7.72(2 \mathrm{H}, \mathrm{d}, J$ $=8.1 \mathrm{~Hz}) .{ }^{13} \mathrm{C}$ NMR $\left(\mathrm{CDCl}_{3}\right) \delta 21.5,23.6,34.3,36.3,42.2,127.0,129.8,136.7,143.6,201.7$. ESIMS: $m / z 336\left(\mathrm{M}^{+}+1\right)$, 334 $\left(\mathrm{M}^{+}+1\right)$. HRMS calcd for $\mathrm{C}_{12} \mathrm{H}_{16} \mathrm{BrNO}_{3} \mathrm{SNa}:\left(\mathrm{M}^{+}+\mathrm{Na}\right)$ 355.9945. Found: 355.9927.

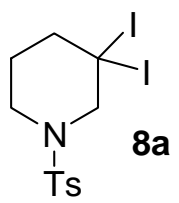

White solid. ${ }^{1} \mathrm{H}$ NMR (300 MHz, $\left.\mathrm{CDCl}_{3}\right) \delta 1.65-1.75(2 \mathrm{H}, \mathrm{m}), 2.33(2 \mathrm{H}, \mathrm{t}, J=5.7 \mathrm{~Hz}), 2.45(3 \mathrm{H}, \mathrm{s})$, $3.08(2 \mathrm{H}, \mathrm{t}, J=4.5 \mathrm{~Hz}), 3.40(2 \mathrm{H}, \mathrm{s}), 7.34(2 \mathrm{H}, \mathrm{d}, J=8.1 \mathrm{~Hz}), 7.66(2 \mathrm{H}, \mathrm{d}, J=8.1 \mathrm{~Hz}) .{ }^{13} \mathrm{C}$ NMR $\left(\mathrm{CDCl}_{3}\right) \delta 1.61,21.6,26.1,45.4,50.9,66.1,127.5,129.8,133.8,144.0$. EIMS: $m / z$ (rel intensity) 491 ( $\left.\mathrm{M}^{+}, 0.3\right), 364$ (41), 237 (100), 210 (4), 181 (3), 155 (10), 91 (69), 82 (43). Anal. calcd for $\mathrm{C}_{12} \mathrm{H}_{15} \mathrm{I}_{2} \mathrm{NO}_{2} \mathrm{~S}: \mathrm{C}, 29.35 ; \mathrm{H}, 3.08 ; \mathrm{N}, 2.85$. Found: C, 29.55; H, 3.17; N, 2.74. 


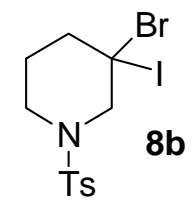

White solid. Mp: $120-122{ }^{\circ} \mathrm{C} .{ }^{1} \mathrm{H}$ NMR $\left(300 \mathrm{MHz}, \mathrm{CDCl}_{3}\right) \delta$ 1.70-1.93 (2H, m), 2.20-2.31 (1H, m), $2.44(3 \mathrm{H}, \mathrm{s}), 2.44-2.53(1 \mathrm{H}, \mathrm{m}), 2.99-3.17(2 \mathrm{H}, \mathrm{m}), 3.33(1 \mathrm{H}, \mathrm{d}, J=12.6 \mathrm{~Hz}), 3.70(1 \mathrm{H}, \mathrm{d}, J=12.6$ $\mathrm{Hz}), 7.34(2 \mathrm{H}, \mathrm{d}, J=8.4 \mathrm{~Hz}), 7.65(2 \mathrm{H}, \mathrm{d}, J=8.4 \mathrm{~Hz}) .{ }^{13} \mathrm{C} \mathrm{NMR}\left(\mathrm{CDCl}_{3}\right) \delta$ 21.5, 25.1, 33.5, 45.3, 49.0, 64.3, 127.5, 129.8, 134.1, 144.0. EIMS: $m / z$ (rel intensity) $444\left(\mathrm{M}^{+}\left({ }^{81} \mathrm{Br}\right)-1,0.3\right), 442\left(\mathrm{M}^{+}\left({ }^{79} \mathrm{Br}\right)-1,0.2\right)$, 318 (64), 316 (67), 237 (6), 207 (4), 155 (73), 91 (100). Anal. calcd for $\mathrm{C}_{12} \mathrm{H}_{15} \mathrm{BrINO}_{2} \mathrm{~S}: \mathrm{C}, 32.45$; H, 3.40; N, 3.15. Found: C, 32.42; H, 3.47; N, 3.03.

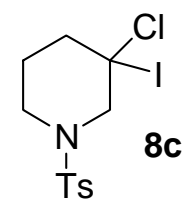

White solid. Mp: 120-122 ${ }^{\circ} \mathrm{C} .{ }^{1} \mathrm{H}$ NMR $\left(300 \mathrm{MHz}, \mathrm{CDCl}_{3}\right) \delta$ 1.68-1.80 (1H, m), 1.89-2.00 (1H, m), 2.10-2.19 (1H, m), $2.45(3 \mathrm{H}, \mathrm{s}), 2.45-2.54(1 \mathrm{H}, \mathrm{m}), 3.08(2 \mathrm{H}, \mathrm{t}, J=5.7 \mathrm{~Hz}), 3.31(1 \mathrm{H}, \mathrm{d}, J=12.3 \mathrm{~Hz})$, $3.69(1 \mathrm{H}, \mathrm{d}, J=12.3 \mathrm{~Hz}), 7.34(2 \mathrm{H}, \mathrm{d}, J=8.1 \mathrm{~Hz}), 7.66(2 \mathrm{H}, \mathrm{d}, J=8.1 \mathrm{~Hz}) .{ }^{13} \mathrm{C} \mathrm{NMR}\left(\mathrm{CDCl}_{3}\right) \delta 21.6$, 24.4, 45.3, 48.0, 48.1, 63.6, 127.5, 129.8, 134.1, 143.9. EIMS: $m / z$ (rel intensity) $400\left(\mathrm{M}^{+}+1,2\right), 364$ (0.6), 304 (3), 272 (37), 237 (2), 155 (29), 91 (100), 65 (20). Anal. calcd for $\mathrm{C}_{12} \mathrm{H}_{15} \mathrm{ClINO}_{2} \mathrm{~S}$ : C, 36.06; H, 3.78; N, 3.50. Found: C, 36.27; H, 4.07; N, 3.41.

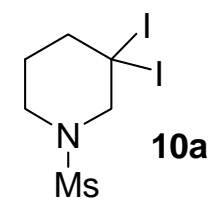

White solid. Mp: 132-134 ${ }^{\circ} \mathrm{C} .{ }^{1} \mathrm{H}$ NMR $\left(300 \mathrm{MHz}, \mathrm{CDCl}_{3}\right) \delta 1.70-1.78(2 \mathrm{H}, \mathrm{m}), 2.44(2 \mathrm{H}, \mathrm{t}, J=5.4$ $\mathrm{Hz}), 2.96(3 \mathrm{H}, \mathrm{s}), 3.33(2 \mathrm{H}, \mathrm{t}, J=5.4 \mathrm{~Hz}), 3.64(2 \mathrm{H}, \mathrm{s}) .{ }^{13} \mathrm{C} \mathrm{NMR}\left(\mathrm{CDCl}_{3}\right) \delta$ 2.6, 26.4, 38.4, 45.1, 51.1, 66.1. EIMS: $m / z$ (rel intensity) $416\left(\mathrm{M}^{+}+1,0.2\right), 288$ (27), 181 (6), 161 (100),151 (3), 127 (3), 82 (61), 55 (47). Anal. calcd for $\mathrm{C}_{6} \mathrm{H}_{11} \mathrm{I}_{2} \mathrm{NO}_{2} \mathrm{~S}$ : C, 17.36; H, 2.67; N, 3.37. Found: C, 17.47; H, 2.82; N, 3.26. 


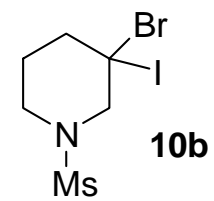

White solid. Mp: $116-118{ }^{\circ} \mathrm{C} .{ }^{1} \mathrm{H}$ NMR $\left(300 \mathrm{MHz}, \mathrm{CDCl}_{3}\right) \delta$ 1.72-1.82 (1H, m), 1.87-2.00 (1H, m), 2.35-2.43 (1H, m), 2.57-2.66 (1H, m), $2.95(3 \mathrm{H}, \mathrm{s}), 3.30-3.40(2 \mathrm{H}, \mathrm{m}), 3.66(1 \mathrm{H}, \mathrm{d}, J=13.5 \mathrm{~Hz}), 3.90$ $(1 \mathrm{H}, \mathrm{d}, J=13.5 \mathrm{~Hz}) .{ }^{13} \mathrm{C} \mathrm{NMR}\left(\mathrm{CDCl}_{3}\right) \delta 25.4,34.3,38.5,44.9,49.1,64.1$. EIMS: $\mathrm{m} / z$ (rel intensity) $242\left(\mathrm{M}^{+}\left({ }^{81} \mathrm{Br}\right)-\mathrm{I}, 100\right), 240\left(\mathrm{M}^{+}\left({ }^{79} \mathrm{Br}\right)-\mathrm{I}, 82\right), 161$ (60), 133 (16), 108 (12), 83 (68), 68 (8), 55 (86). Anal. calcd for $\mathrm{C}_{6} \mathrm{H}_{11} \mathrm{BrINO}_{2} \mathrm{~S}$ : C, 19.58; H, 3.01; N, 3.81. Found: C, 19.64; H, 3.27; N, 3.66.

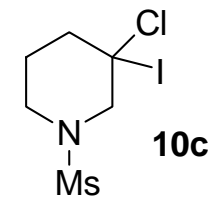

White solid. Mp: 92-94 ${ }^{\circ} \mathrm{C} .{ }^{1} \mathrm{H}$ NMR $\left(300 \mathrm{MHz}, \mathrm{CDCl}_{3}\right) \delta$ 1.68-1.80 (1H, m), 1.92-2.10 (1H, m), 2.26$2.37(1 \mathrm{H}, \mathrm{m}), 2.53-2.66(1 \mathrm{H}, \mathrm{m}), 2.95(3 \mathrm{H}, \mathrm{s}), 3.22-3.34(1 \mathrm{H}, \mathrm{m}), 3.38-3.47(1 \mathrm{H}, \mathrm{m}), 3.67(1 \mathrm{H}, \mathrm{d}, J=$ $13.5 \mathrm{~Hz}), 3.91(1 \mathrm{H}, \mathrm{d}, J=13.5 \mathrm{~Hz}) .{ }^{13} \mathrm{C} \mathrm{NMR}\left(\mathrm{CDCl}_{3}\right) \delta 24.6,38.7,44.8,48.1,48.6,63.4$. EIMS: $\mathrm{m} / z$ (rel intensity) $324\left(\mathrm{M}^{+}+1,1\right), 196$ (63), 160 (6), 127 (15), 118 (83), 89 (75), 82 (86), 55 (100). Anal. calcd for $\mathrm{C}_{6} \mathrm{H}_{11} \mathrm{ClINO}_{2} \mathrm{~S}: \mathrm{C}, 22.27 ; \mathrm{H}, 3.43 ; \mathrm{N}, 4.33$. Found: C, 22.51; H, 3.53; N, 4.19.

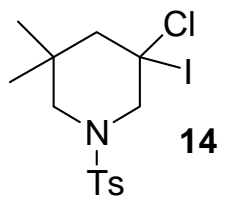

White solid. Mp: 110-112 ${ }^{\circ} \mathrm{C} .{ }^{1} \mathrm{H}$ NMR $\left(300 \mathrm{MHz}, \mathrm{CDCl}_{3}\right) \delta 0.95(3 \mathrm{H}, \mathrm{s}), 1.25(3 \mathrm{H}, \mathrm{s}), 2.36-2.64(6 \mathrm{H}$, m), $3.31(1 \mathrm{H}, \mathrm{d}, J=12.3 \mathrm{~Hz}), 3.44(1 \mathrm{H}, \mathrm{d}, J=12.9 \mathrm{~Hz}), 4.20(1 \mathrm{H}, \mathrm{d}, J=12.9 \mathrm{~Hz}), 7.35(2 \mathrm{H}, \mathrm{d}, J=7.8$ $\mathrm{Hz}), 7.68(2 \mathrm{H}, \mathrm{d}, J=7.8 \mathrm{~Hz}) .{ }^{13} \mathrm{C} \mathrm{NMR}\left(\mathrm{CDCl}_{3}\right) \delta 21.5,26.6,29.0,35.5,45.2,56.9,60.0,64.8,127.4$, 129.8, 134.6, 143.9. EIMS: m/z (rel intensity) 300 ( $\left.\mathrm{M}^{+}-\mathrm{I}, 35\right), 252$ (4), 167 (2), 155 (51), 144 (5), 108 (7), 91 (100), 65 (29). Anal. calcd for $\mathrm{C}_{14} \mathrm{H}_{19} \mathrm{ClINO}_{2} \mathrm{~S}$ : C, 39.31; H, 4.48; N, 3.27. Found: C, 39.38; H, 4.56; N, 3.12. 


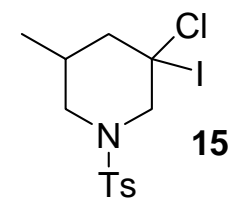

White solid. Mp: $134-136{ }^{\circ} \mathrm{C} .{ }^{1} \mathrm{H}$ NMR $\left(300 \mathrm{MHz}, \mathrm{CDCl}_{3}\right) \delta 0.88 / 0.95(3 \mathrm{H}, 2 \mathrm{~d}, J=6.3 \mathrm{~Hz}), 1.16-$ 1.25/1.85-2.42 (3H, 2m), 2.17/3.17 (1H, $2 \mathrm{~d}, J=12.6 \mathrm{~Hz}), 2.46(3 \mathrm{H}, \mathrm{s}), 2.58 / 2.82(1 \mathrm{H}, 2 \mathrm{~d}, J=14.4 \mathrm{~Hz})$, 3.74-3.91 (1H, m), 4.19-4.25/4.41-4.47 (1H, 2m), $7.36(2 \mathrm{H}, \mathrm{d}, J=8.1 \mathrm{~Hz}), 7.69(2 \mathrm{H}, \mathrm{d}, J=8.1 \mathrm{~Hz})$. ${ }^{13} \mathrm{C}$ NMR $\left(\mathrm{CDCl}_{3}\right) \delta 17.3 / 17.4,21.6,31.2 / 29.7,46.2 / 48.7,51.9 / 51.8,56.0 / 56.4,63.4 / 63.1,127.5 / 127.4$, 129.9/129.8, 134.4/134.1, 143.8/143.0. EIMS: $m / z$ (rel intensity) 286 (M+I, 50), 251 (0.7), 184 (1), 155 (59), 131 (4), 91 (100), 65 (21), 41 (20). Anal. calcd for $\mathrm{C}_{13} \mathrm{H}_{17} \mathrm{ClINO}_{2} \mathrm{~S}: \mathrm{C}, 37.74 ; \mathrm{H}, 4.14$; N, 3.39. Found: C, 37.77; H, 4.31; N, 3.24.

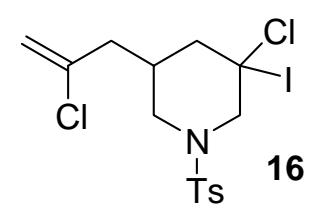

White solid. ${ }^{1} \mathrm{H}$ NMR $\left(300 \mathrm{MHz}, \mathrm{CDCl}_{3}\right) \delta 1.25(1 \mathrm{H}, \mathrm{dd}, J=11.7,13.5 \mathrm{~Hz}), 1.99-2.40(3 \mathrm{H}, \mathrm{m})$, 2.28/3.21 (1H, 2d, $J=12.6 \mathrm{~Hz}), 2.45(3 \mathrm{H}, \mathrm{s}), 2.55-2.66 / 3.17-3.26(2 \mathrm{H}, 2 \mathrm{~m}), 3.73-3.97(1 \mathrm{H}, \mathrm{m})$, 4.18/4.43 (1H, 2d, $J=16.5 / 12.9 \mathrm{~Hz}), 5.15(1 \mathrm{H}, \mathrm{s}), 5.27(1 \mathrm{H}, \mathrm{s}), 7.33 / 7.34(2 \mathrm{H}, 2 \mathrm{~d}, J=8.1 \mathrm{~Hz}), 7.67$ $(2 \mathrm{H}, \mathrm{d}, J=8.1 \mathrm{~Hz}) .{ }^{13} \mathrm{C}$ NMR $\left(\mathrm{CDCl}_{3}\right) \delta 21.5,32.4 / 34.0,41.6 / 41.8,45.2 / 47.6,49.7 / 49.9,53.2 / 53.7$, 63.3/63.7, 114.9/115.0, 127.4/127.6, 129.8/129.9, 134.1/134.5, 138.4/138.5, 143.9/144.1. ESIMS: $\mathrm{m} / \mathrm{z}$ $474.0\left(\mathrm{M}^{+}+1\right)$. HRMS calcd for $\mathrm{C}_{15} \mathrm{H}_{19} \mathrm{Cl}_{2} \mathrm{INO}_{2} \mathrm{~S}\left(\mathrm{M}^{+}+1\right)$ : 473.9554. Found: 473.9553 .

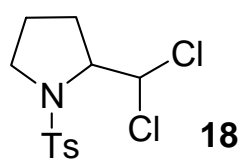

White solid. Mp: 80-82 ${ }^{\circ} \mathrm{C} .{ }^{1} \mathrm{H}$ NMR (300 MHz, $\left.\mathrm{CDCl}_{3}\right) \delta$ 1.41-1.52 (1H, m), 1.88-2.04 (2H, m), 2.19$2.28(1 \mathrm{H}, \mathrm{m}), 2.45(3 \mathrm{H}, \mathrm{s}), 3.27-3.36(1 \mathrm{H}, \mathrm{m}), 3.43-3.52(1 \mathrm{H}, \mathrm{m}), 4.01-4.06(1 \mathrm{H}, \mathrm{m}), 6.28(1 \mathrm{H}, \mathrm{dd}, J=$ 0.9, $2.7 \mathrm{~Hz}), 7.36(2 \mathrm{H}, \mathrm{d}, J=8.4 \mathrm{~Hz}), 7.74(2 \mathrm{H}, \mathrm{d}, J=8.4 \mathrm{~Hz}) \cdot{ }^{13} \mathrm{C} \mathrm{NMR}\left(\mathrm{CDCl}_{3}\right) \delta 21.4,24.3,26.3$, 50.1, 65.4, 75.5, 127.3, 129.8, 133.6, 144.0. EIMS: $\mathrm{m} / z$ (rel intensity) $307\left(\mathrm{M}^{+}, 0.1\right), 272(0.3), 224$ (83), 155 (47), 139 (2), 91 (100), 65 (22), 41 (14). Anal. calcd for $\mathrm{C}_{12} \mathrm{H}_{15} \mathrm{Cl}_{2} \mathrm{NO}_{2} \mathrm{~S}$ : C, 46.76; $\mathrm{H}, 4.91$; N, 4.54. Found: C, 46.95; H, 4.91; N, 4.34. 


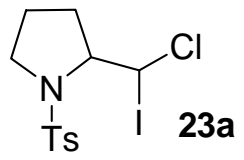

White solid. Mp: $112-114{ }^{\circ} \mathrm{C} .{ }^{1} \mathrm{H}$ NMR $\left(300 \mathrm{MHz}, \mathrm{CDCl}_{3}\right) \delta$ 1.33-1.48 (1H, m), 1.91-2.08/2.21-2.31 $(3 \mathrm{H}, 2 \mathrm{~m}), 2.45(3 \mathrm{H}, \mathrm{s}), 3.12-3.16 / 4.17-4.25(1 \mathrm{H}, 2 \mathrm{~m}), 3.39-3.51(2 \mathrm{H}, \mathrm{m}), 6.28 / 6.32(1 \mathrm{H}, 2 \mathrm{~d}, J=$ 2.4/3.0 Hz), $7.35(2 \mathrm{H}, \mathrm{d}, J=8.1 \mathrm{~Hz}), 7.71 / 7.74(2 \mathrm{H}, 2 \mathrm{~d}, J=8.1 \mathrm{~Hz}) .{ }^{13} \mathrm{C} \mathrm{NMR}\left(\mathrm{CDCl}_{3}\right) \delta 21.6$, 24.2/24.6, 28.9/30.8, 35.7/40.6, 50.3/51.2, 65.8/67.5, 127.5/127.6, 129.9, 134.3, 144.0/144.2. EIMS: m/z (rel intensity) 399 ( $\mathrm{M}^{+}, 0.9$ ), 376 (0.7), 272 (2), 224 (100), 205 (11), 155 (41), 125 (11), 91 (68). Anal. calcd for $\mathrm{C}_{12} \mathrm{H}_{15} \mathrm{ClINO}_{2} \mathrm{~S}$ : C, 36.06; H, 3.78; N, 3.50. Found: C, 36.27; H, 3.85; N, 3.42

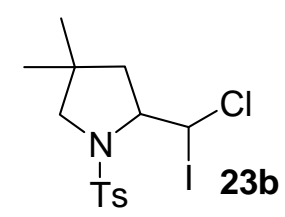

White solid. ${ }^{1} \mathrm{H}$ NMR (300 MHz, $\left.\mathrm{CDCl}_{3}\right) \delta 0.47 / 0.54(3 \mathrm{H}, 2 \mathrm{~s}), 1.07 / 1.08(3 \mathrm{H}, 2 \mathrm{~s}), 1.80-2.14(2 \mathrm{H}, \mathrm{m})$, $2.44(3 \mathrm{H}, \mathrm{s}), 3.13-3.42 / 4.32-4.39$ (3H, $2 \mathrm{~m}), 6.40 / 6.49(1 \mathrm{H}, 2 \mathrm{~d}, J=3.0 \mathrm{~Hz}), 7.32 / 7.33(2 \mathrm{H}, 2 \mathrm{~d}, J=8.1$ $\mathrm{Hz}), 7.71-7.77(2 \mathrm{H}, \mathrm{m}) .{ }^{13} \mathrm{C}$ NMR $\left(\mathrm{CDCl}_{3}\right) \delta 21.6,25.3 / 25.4,26.0 / 26.6,35.0 / 37.7,38.1 / 45.1$, 42.7/42.8, 61.6/62.6, 65.5/67.0, 127.3/127.5, 129.8, 135.6/136.0, 143.8/144.0. ESIMS: $\mathrm{m} / z \quad 428.0$ $\left(\mathrm{M}^{+}+1\right)$. HRMS calcd for $\mathrm{C}_{14} \mathrm{H}_{19} \mathrm{ClINO}_{2} \mathrm{SNa}:\left(\mathrm{M}^{+}+\mathrm{Na}\right)$ 449.9769. Found: 449.9762 .

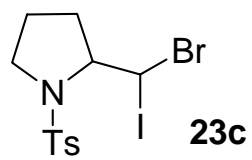

White solid. Mp: $116-118{ }^{\circ} \mathrm{C} .{ }^{1} \mathrm{H}$ NMR $\left(300 \mathrm{MHz}, \mathrm{CDCl}_{3}\right) \delta$ 1.30-1.47 $(1 \mathrm{H}, \mathrm{m}), 1.93-2.22(3 \mathrm{H}, \mathrm{m})$, $2.45(3 \mathrm{H}, \mathrm{s}), 3.20-3.28 / 4.01-4.06(1 \mathrm{H}, 2 \mathrm{~m}), 3.43-3.53(2 \mathrm{H}, \mathrm{m}), 6.08-6.11(1 \mathrm{H}, \mathrm{m}), 7.35(2 \mathrm{H}, \mathrm{d}, J=8.1$ $\mathrm{Hz}), 7.72(1 \mathrm{H}, \mathrm{d}, J=8.1 \mathrm{~Hz}), 7.73(1 \mathrm{H}, \mathrm{d}, J=8.1 \mathrm{~Hz}) .{ }^{13} \mathrm{C} \mathrm{NMR}\left(\mathrm{CDCl}_{3}\right) \delta 20.9 / 24.0,21.6,24.3 / 24.6$, 30.3/31.6, 50.6/51.2, 65.9/67.2, 127.5/127.6, 129.9, 134.4, 144.1. ESIMS: $m / z$ 445.8 $\left(\mathrm{M}^{+}\left({ }^{81} \mathrm{Br}\right)+1\right)$, $443.8\left(\mathrm{M}^{+}\left({ }^{79} \mathrm{Br}\right)+1\right)$. Anal. calcd for $\mathrm{C}_{12} \mathrm{H}_{15} \mathrm{BrINO}_{2} \mathrm{~S}$ : C, 32.45; H, 3.40; N, 3.15. Found: C, 32.63; $\mathrm{H}$, $3.49 ; \mathrm{N}, 3.07$. 


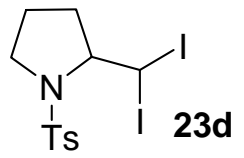

White solid. Mp: 130-132 ${ }^{\circ} \mathrm{C} .{ }^{1} \mathrm{H}$ NMR $\left(300 \mathrm{MHz}, \mathrm{CDCl}_{3}\right) \delta$ 1.33-1.45 (1H, m), 1.85-2.04 (2H, m), 2.17-2.30 (1H, m), $2.45(3 \mathrm{H}, \mathrm{s}), 3.24-3.30(1 \mathrm{H}, \mathrm{m}), 3.49-3.54(2 \mathrm{H}, \mathrm{m}), 5.71(1 \mathrm{H}, \mathrm{d}, J=2.7 \mathrm{~Hz}), 7.35$ $(2 \mathrm{H}, \mathrm{d}, J=8.1 \mathrm{~Hz}), 7.72(2 \mathrm{H}, \mathrm{d}, J=8.1 \mathrm{~Hz}) .{ }^{13} \mathrm{C} \mathrm{NMR}\left(\mathrm{CDCl}_{3}\right) \delta-10.5,21.6,24.4,32.9,51.3,66.8$, 127.5, 129.9, 134.4, 144.1. ESIMS: $m / z 491.8\left(\mathrm{M}^{+}+\mathrm{H}\right)$. Anal. calcd for $\mathrm{C}_{12} \mathrm{H}_{15} \mathrm{I}_{2} \mathrm{NO}_{2} \mathrm{~S}: \mathrm{C}, 29.35 ; \mathrm{H}$, 3.08; N, 2.85. Found: C, 29.62; H, 3.13; N, 2.68.

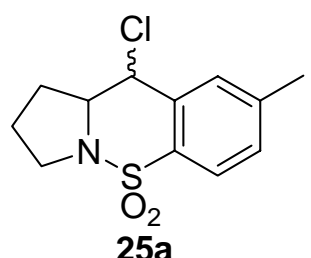

White solid. ${ }^{1} \mathrm{H}$ NMR $\left(300 \mathrm{MHz}, \mathrm{CDCl}_{3}\right) \delta$ 1.78-2.10 (3H, m), 2.18-2.29/2.47-2.59 (1H, 2m), 2.43/2.45 (3H, 2s), 3.17-3.25/3.66-3.74 (1H, 2m), 3.48-3.63 (1H, m), 4.13-4.22/4.29-4.36 (1H, 2m), 4.93/5.46 $(1 \mathrm{H}, 2 \mathrm{~d}, J=10.2 / 4.5 \mathrm{~Hz}), 7.29 / 7.32(1 \mathrm{H}, 2 \mathrm{~d}, J=8.1 \mathrm{~Hz}), 7.42 / 7.52(1 \mathrm{H}, 2 \mathrm{~s}), 7.75(1 \mathrm{H}, \mathrm{d}, J=8.1 \mathrm{~Hz})$. ${ }^{13} \mathrm{C}$ NMR $\left(\mathrm{CDCl}_{3}\right) \delta 21.6,22.9 / 23.5,32.1 / 29.5,45.7 / 48.2,58.7 / 57.0,64.7 / 62.7$, 123.8/124.4, 129.8/129.6, 129.9/129.8, 134.1/133.8, 134.9/134.6, 143.3/143.4. EIMS: $m / z$ (rel intensity) $271\left(\mathrm{M}^{+}\right.$, 21), 236 (4), 172 (100), 157 (8), 138 (22), 115 (10), 103 (39), 77 (17). HRMS calcd for $\mathrm{C}_{2} \mathrm{H}_{15} \mathrm{ClNO}_{2} \mathrm{~S}:\left(\mathrm{M}^{+}+1\right)$ 272.0499. Found: 272.0507 .

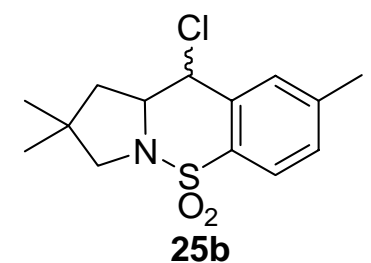

White solid. Mp: $124-126{ }^{\circ} \mathrm{C} .{ }^{1} \mathrm{H}$ NMR $\left(300 \mathrm{MHz}, \mathrm{CDCl}_{3}\right) \delta$ 1.04/1.20 (3H, 2s), 1.17/1.22 (3H, 2s), 1.51-1.59/2.27-2.35 (1H, 2m), 1.81-1.95 (1H, m), 2.43/2.46 (3H, 2s), 2.72/3.23 (1H, 2d, $J=9.6 \mathrm{~Hz})$, 3.33/3.47 (1H, 2d, $J=9.6 \mathrm{~Hz}), 4.22-4.31 / 4.43-4.51(1 \mathrm{H}, 2 \mathrm{~m}), 4.96 / 5.55(1 \mathrm{H}, 2 \mathrm{~d}, J=10.5 / 5.4 \mathrm{~Hz})$, 7.26-7.33 (1H, m), 7.47-7.51 (1H, m), 7.72-7.77 (1H, m). ${ }^{13} \mathrm{C} \mathrm{NMR}\left(\mathrm{CDCl}_{3}\right) \delta$ 21.7/21.6, 26.2/25.8, 
26.9/27.2, 36.8/37.6, 43.0/46.5, 57.7/56.3, 61.0/59.6, 62.9/64.6, 123.8/124.6, 129.3/129.5, 129.6/129.8, 133.9/133.5, 135.0, 143.4/143.2. EIMS: $m / z$ (rel intensity) $299\left(\mathrm{M}^{+}, 22\right), 264$ (4), 220 (4), 200 (100), 167 (9), 144 (18), 139 (22), 103 (32). Anal. calcd for $\mathrm{C}_{14} \mathrm{H}_{18} \mathrm{ClNO}_{2} \mathrm{~S}: \mathrm{C}, 56.08 ; \mathrm{H}, 6.05 ; \mathrm{N}$, 4.67. Found: C, 56.07; H, 5.98; N, 4.47.

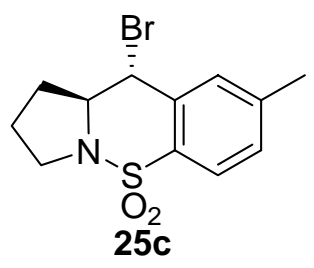

White solid. Mp: $128-130{ }^{\circ} \mathrm{C} .{ }^{1} \mathrm{H}$ NMR $\left(300 \mathrm{MHz}, \mathrm{CDCl}_{3}\right) \delta$ 1.96-2.10 (3H, m), $2.42(3 \mathrm{H}, \mathrm{s}), 2.51-2.62$ $(1 \mathrm{H}, \mathrm{m}), 3.47-3.56(1 \mathrm{H}, \mathrm{m}), 3.67-3.75(1 \mathrm{H}, \mathrm{m}), 4.29-4.40(1 \mathrm{H}, \mathrm{m}), 5.07(1 \mathrm{H}, \mathrm{d}, J=10.2 \mathrm{~Hz}), 7.27(1 \mathrm{H}$, $\mathrm{d}, J=8.1 \mathrm{~Hz}), 7.53(1 \mathrm{H}, \mathrm{s}), 7.74(1 \mathrm{H}, \mathrm{d}, J=8.1 \mathrm{~Hz}) .{ }^{13} \mathrm{C} \mathrm{NMR}\left(\mathrm{CDCl}_{3}\right) \delta 21.5,22.7,33.0,46.0,49.6$, 65.0, 123.9, 129.9, 131.7, 134.4, 135.0, 143.2. EIMS: $\mathrm{m} / \mathrm{z}$ (rel intensity) $317\left(\mathrm{M}^{+}\left({ }^{81} \mathrm{Br}\right), 10\right), 315$ $\left(\mathrm{M}^{+}\left({ }^{79} \mathrm{Br}\right), 10\right), 236$ (46), 172 (100), 157 (14), 144 (17), 115 (15), 103 (54). HRMS calcd for $\mathrm{C}_{12} \mathrm{H}_{15} \mathrm{BrNO}_{2} \mathrm{~S}:\left(\mathrm{M}^{+}+1\right)$ 315.9989. Found: 316.0001 .

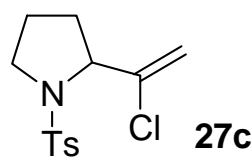

White solid. ${ }^{1} \mathrm{H}$ NMR $\left(300 \mathrm{MHz}, \mathrm{CDCl}_{3}\right) \delta$ 1.64-2.05 (4H, m), $2.43(3 \mathrm{H}, \mathrm{s}), 3.27-3.33(1 \mathrm{H}, \mathrm{m}), 3.47-$ $3.50(1 \mathrm{H}, \mathrm{m}), 4.31-4.35(1 \mathrm{H}, \mathrm{m}), 5.33(1 \mathrm{H}, \mathrm{s}), 5.57(1 \mathrm{H}, \mathrm{s}), 7.32(2 \mathrm{H}, \mathrm{d}, J=8.1 \mathrm{~Hz}), 7.73(2 \mathrm{H}, \mathrm{d}, J=$ $8.1 \mathrm{~Hz}) .{ }^{13} \mathrm{C}$ NMR $\left(\mathrm{CDCl}_{3}\right) \delta 21.5,23.8,31.1,49.1,64.3,113.6,127.5,129.7,135.2,141.8,143.6$. EIMS: $m / z$ (rel intensity) $285\left(\mathrm{M}^{+}, 2\right), 224$ (57), 155 (41), 130 (11), 102 (9), 91 (100), 65 (29), 41 (19). HRMS calcd for $\mathrm{C}_{13} \mathrm{H}_{17} \mathrm{ClNO}_{2} \mathrm{~S}$ : $\left(\mathrm{M}^{+}+1\right)$ 286.0664. Found: 286.0663 .

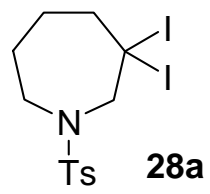

White solid. ${ }^{1} \mathrm{H}$ NMR $\left(300 \mathrm{MHz}, \mathrm{CDCl}_{3}\right) \delta 1.43-1.52(2 \mathrm{H}, \mathrm{m}), 1.72-1.81(2 \mathrm{H}, \mathrm{m}), 2.44(3 \mathrm{H}, \mathrm{s}), 2.78$ $(2 \mathrm{H}, \mathrm{d}, J=5.1 \mathrm{~Hz}), 3.17(2 \mathrm{H}, \mathrm{d}, J=6.6 \mathrm{~Hz}), 3.87(2 \mathrm{H}, \mathrm{s}), 7.32(2 \mathrm{H}, \mathrm{d}, J=8.1 \mathrm{~Hz}), 7.70(2 \mathrm{H}, \mathrm{d}, J=8.1$ 
$\mathrm{Hz}) .{ }^{13} \mathrm{C} \mathrm{NMR}\left(\mathrm{CDCl}_{3}\right) \delta 14.4,21.6,26.4,27.0,49.6,57.1,68.2,127.5,129.8,135.4,143.8$. EIMS: m/z (rel intensity) 377 (M+HI, 1), 333 (17), 319 (12), 302 (17), 256 (14), 202 (40), 103 (100), 77 (47). Anal. calcd for $\mathrm{C}_{13} \mathrm{H}_{17} \mathrm{I}_{2} \mathrm{NO}_{2} \mathrm{~S}: \mathrm{C}, 30.91 ; \mathrm{H}, 3.39 ; \mathrm{N}, 2.77$. Found: C, 31.11; H, 3.48; N, 2.43.

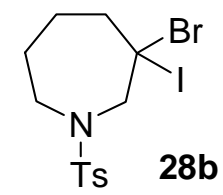

White solid. ${ }^{1} \mathrm{H}$ NMR $\left(300 \mathrm{MHz}, \mathrm{CDCl}_{3}\right) \delta$ 1.50-1.58 (1H, m), 1.68-1.80 (3H, m), 2.44 (3H, s), 2.65$2.73(1 \mathrm{H}, \mathrm{m}), 2.82-2.86(1 \mathrm{H}, \mathrm{m}), 3.10-3.18(1 \mathrm{H}, \mathrm{m}), 3.21-3.29(1 \mathrm{H}, \mathrm{m}), 3.95(1 \mathrm{H}, \mathrm{d}, J=15.0 \mathrm{~Hz}), 4.05$ $(1 \mathrm{H}, \mathrm{d}, J=15.0 \mathrm{~Hz}), 7.32(2 \mathrm{H}, \mathrm{d}, J=8.1 \mathrm{~Hz}), 7.71(2 \mathrm{H}, \mathrm{d}, J=8.1 \mathrm{~Hz}) .{ }^{13} \mathrm{C} \mathrm{NMR}\left(\mathrm{CDCl}_{3}\right) \delta 21.5,24.8$, 27.3, 43.5, 49.8, 54.6, 66.9, 127.5, 129.8, 135.6, 143.7. ESIMS: $m / z 457.9\left(\mathrm{M}^{+}+1\right)$. Anal. calcd for $\mathrm{C}_{13} \mathrm{H}_{17} \mathrm{BrINO}_{2} \mathrm{~S}: \mathrm{C}, 34.08 ; \mathrm{H}, 3.74 ; \mathrm{N}, 3.06$. Found: C, 34.21; H, 3.89; N, 2.83.

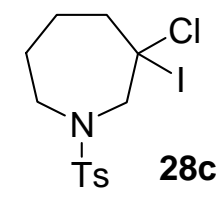

White solid. Mp: $104-106{ }^{\circ} \mathrm{C} .{ }^{1} \mathrm{H}$ NMR $\left(300 \mathrm{MHz}, \mathrm{CDCl}_{3}\right) \delta 1.45-1.52(1 \mathrm{H}, \mathrm{m}), 1.69-1.86(3 \mathrm{H}, \mathrm{m})$, $2.44(3 \mathrm{H}, \mathrm{s}), 2.55-2.64(1 \mathrm{H}, \mathrm{m}), 2.75-2.84(1 \mathrm{H}, \mathrm{m}), 2.97-3.06(1 \mathrm{H}, \mathrm{m}), 3.35-3.42(1 \mathrm{H}, \mathrm{m}), 3.93(1 \mathrm{H}, \mathrm{d}$, $J=15.0 \mathrm{~Hz}), 4.10(1 \mathrm{H}, \mathrm{d}, J=15.0 \mathrm{~Hz}), 7.32(2 \mathrm{H}, \mathrm{d}, J=8.1 \mathrm{~Hz}), 7.71(2 \mathrm{H}, \mathrm{d}, J=8.1 \mathrm{~Hz}) .{ }^{13} \mathrm{C}$ NMR $\left(\mathrm{CDCl}_{3}\right) \delta 21.5,23.6,27.6,49.7,53.4,57.0,66.4,127.4,129.8,135.7,143.7$. EIMS: $\mathrm{m} / z$ (rel intensity) 286 ( $\mathrm{M}^{+}$-I, 42), 155 (51), 139 (5), 130 (11), 96 (16), 91 (100), 65 (25), 42 (26). Anal. calcd for $\mathrm{C}_{13} \mathrm{H}_{17} \mathrm{ClINO}_{2} \mathrm{~S}: \mathrm{C}, 37.74 ; \mathrm{H}, 4.14 ; \mathrm{N}, 3.39$. Found: C, 37.78; H, 3.93; N, 3.18.

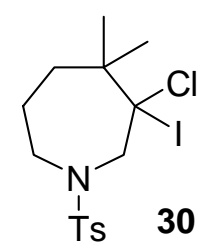

White solid. ${ }^{1} \mathrm{H}$ NMR $\left(300 \mathrm{MHz}, \mathrm{CDCl}_{3}\right) \delta 1.23(3 \mathrm{H}, \mathrm{s}), 1.31(3 \mathrm{H}, \mathrm{s}), 1.53-1.80$ (3H, m), 1.94-2.05 $(1 \mathrm{H}, \mathrm{m}), 2.43(3 \mathrm{H}, \mathrm{s}), 3.01-3.11(1 \mathrm{H}, \mathrm{m}), 3.35-3.45(1 \mathrm{H}, \mathrm{m}), 3.92(1 \mathrm{H}, \mathrm{d}, J=15.0 \mathrm{~Hz}), 4.29(1 \mathrm{H}, \mathrm{d}, J=$ $15.0 \mathrm{~Hz}), 7.32(2 \mathrm{H}, \mathrm{d}, J=8.1 \mathrm{~Hz}), 7.70(2 \mathrm{H}, \mathrm{d}, J=8.1 \mathrm{~Hz}) .{ }^{13} \mathrm{C} \mathrm{NMR}\left(\mathrm{CDCl}_{3}\right) \delta 21.6,21.8,29.0,29.8$, 
32.3, 46.9, 47.6, 62.0, 127.4, 129.7, 135.9, 143.6. ESIMS: $m / z 442\left(\mathrm{M}^{+}+1\right)$. HRMS calcd for $\mathrm{C}_{15} \mathrm{H}_{22} \mathrm{ClINO}_{2} \mathrm{~S}\left(\mathrm{M}^{+}+1\right)$ : 442.0100. Found: 442.0099 .

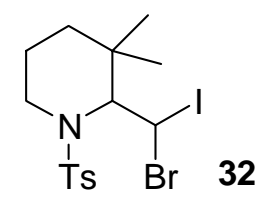

White solid. ${ }^{1} \mathrm{H}$ NMR $\left(300 \mathrm{MHz}, \mathrm{CDCl}_{3}\right) \delta$ 0.96/1.04 (3H, 2s), 1.13-1.26 (1H, m), 1.28/1.36 (3H, 2s), 1.42-1.53 (1H, m), 1.64-1.87 (1H, m), 2.04-2.22 (1H, m), 2.43 (3H, s), 3.46-3.71 (2H, m), 4.44/5.89 $(1 \mathrm{H}, 2 \mathrm{~d}, J=4.2 \mathrm{~Hz}), 4.51 / 5.81(1 \mathrm{H}, 2 \mathrm{~d}, J=3.9 \mathrm{~Hz}), 7.30(2 \mathrm{H}, \mathrm{d}, J=7.8 \mathrm{~Hz}), 7.79 / 7.88(2 \mathrm{H}, 2 \mathrm{~d}, J=$ $8.4 \mathrm{~Hz}) .{ }^{13} \mathrm{C} \mathrm{NMR}\left(\mathrm{CDCl}_{3}\right) \delta$ 9.2/9.6, 20.6/20.8, 21.8, 28.4, 29.2/29.3, 31.9, 35.2/35.6, 41.4/41.9, 67.4/67.8, 127.5/127.8, 129.4/129.6, 137.6/137.9, 143.3/143.4. EIMS: $m / z$ (rel intensity) 359 $\left(\mathrm{M}^{+}\left({ }^{81} \mathrm{Br}\right)-\mathrm{HI}, 0.7\right), 357$ (M+( $\left.\left.{ }^{+79} \mathrm{Br}\right)-\mathrm{HI}, 0.6\right), 266$ (100), 200 (3), 155 (34), 110 (18), 91 (78), 65 (25). Anal. calcd for $\mathrm{C}_{15} \mathrm{H}_{21}$ BrINO$_{2} \mathrm{~S}$ : C, 37.05; H, 4.35; N, 2.88. Found: C, 37.17; H, 4.27; N, 2.54.

\section{References for known compounds}

\begin{tabular}{|c|l|}
\hline Compound & Reference \\
\hline $\mathbf{4}$ & $\begin{array}{l}\text { Larock, R. C.; Yang, H.; Weinreb, S. M.; Herr, R. J. J. Org. Chem. } \\
\mathbf{1 9 9 4 ,} 59 ; \text {; } 172 .\end{array}$ \\
\hline $\mathbf{6 a}$ & Larock, R. C.; Tu, C.; Pace, P. J. Org. Chem. 1998, 636859. \\
\hline 26a & Larock, R. C.; Tu, C.; Pace, P. J. Org. Chem. 1998; 63; 6859. \\
\hline 27a & $\begin{array}{l}\text { Shaw, R. W.; Gallagher, T. J. Chem. Soc. Perkin Trans. 1, 1994, 24, } \\
3549 .\end{array}$ \\
\hline 27b & $\begin{array}{l}\text { Jonasson, C.; Horvath, A.; Baeckvall, J. J. Am. Chem. Soc. 2000, 122, } \\
9600 .\end{array}$ \\
\hline
\end{tabular}




\section{Computational (UB3LYP/6-31G*) Results on the Cyclization of Radicals A}

$\mathrm{A}(\mathrm{R}=\mathrm{H})$

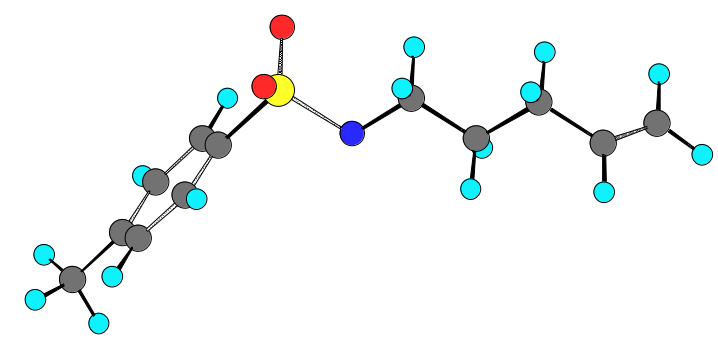

\%chk=A $(\mathbf{R}=\mathbf{H})$-radical

\# ub3lyp/6-31g* opt freq=noraman

calculate for $\mathrm{A}(\mathrm{R}=\mathrm{H})$-radical $\mathrm{HF}=-1070.160748$

02

$\mathrm{C}$

C $\quad 1 \quad 1.529347$

$\begin{array}{lllll}\text { C } & 2 & 1.540128 & 1 & 112.077367\end{array}$ 


$\begin{array}{lcccccc}\mathrm{C} & 3 & 1.504719 & 2 & 112.555643 & 1 & 178.041340 \\ \mathrm{C} & 4 & 1.333216 & 3 & 125.179973 & 2 & 119.181726 \\ \mathrm{~N} & 1 & 1.453659 & 2 & 110.591063 & 3 & -179.696219 \\ \mathrm{~S} & 6 & 1.703932 & 1 & 112.363048 & 2 & -178.913833 \\ \mathrm{O} & 7 & 1.471406 & 6 & 107.641064 & 1 & -67.031648 \\ \mathrm{O} & 7 & 1.471275 & 6 & 108.016380 & 1 & 64.781482 \\ \mathrm{C} & 7 & 1.784831 & 6 & 101.610355 & 1 & 178.871483 \\ \mathrm{C} & 10 & 1.395128 & 7 & 119.453987 & 6 & 90.139758 \\ \mathrm{C} & 11 & 1.393181 & 10 & 118.938541 & 7 & 179.763193 \\ \mathrm{C} & 12 & 1.402146 & 11 & 121.186913 & 10 & -0.022710 \\ \mathrm{C} & 13 & 1.402652 & 12 & 118.507770 & 11 & -0.568559 \\ \mathrm{C} & 14 & 1.392545 & 13 & 121.171437 & 12 & 0.567845 \\ \mathrm{C} & 13 & 1.509917 & 12 & 120.841090 & 11 & 178.696207 \\ \mathrm{H} & 1 & 1.103281 & 2 & 111.092206 & 3 & -58.558133 \\ \mathrm{H} & 1 & 1.102693 & 2 & 111.177129 & 3 & 58.687343 \\ \mathrm{H} & 2 & 1.096307 & 1 & 109.247906 & 6 & 58.448650 \\ \mathrm{H} & 2 & 1.097334 & 1 & 108.928058 & 6 & -57.704046 \\ \mathrm{H} & 3 & 1.098100 & 2 & 109.840242 & 1 & -59.463701 \\ \mathrm{H} & 3 & 1.101299 & 2 & 108.683872 & 1 & 56.728782 \\ \mathrm{H} & 5 & 1.086702 & 4 & 121.818960 & 3 & -179.673795 \\ \mathrm{H} & 5 & 1.088430 & 4 & 121.678727 & 3 & 0.558440 \\ \mathrm{H} & 4 & 1.091600 & 3 & 115.696806 & 2 & -60.156887 \\ \mathrm{H} & 11 & 1.084760 & 10 & 120.013898 & 7 & 0.780311 \\ \mathrm{H} & 12 & 1.087124 & 11 & 119.336010 & 10 & -179.589648 \\ \mathrm{H} & 14 & 1.087187 & 13 & 119.492457 & 12 & -178.974526 \\ \mathrm{H} & 15 & 1.084742 & 14 & 121.061595 & 13 & -178.931024 \\ \mathrm{H} & 1.094603 & 13 & 111.535770 & 12 & 26.394425 \\ \mathrm{H} & 1.094931 & 13 & 111.457496 & 12 & 147.426115 \\ \mathrm{H} & 1698086 & 13 & 110.898920 & 12 & -93.182983\end{array}$


A $(\mathrm{R}=\mathrm{H})$-TS (5-exo)

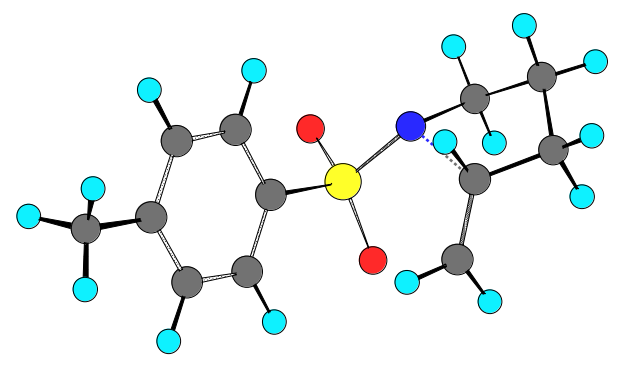

$\%$ chk= A $(\mathbf{R}=\mathbf{H})$-TS (5-exo)

\# ub3lyp/6-31g* opt=(ts,noeigen,readfc,nofreeze) freq=noraman

calculate for A $(\mathrm{R}=\mathrm{H})-\mathrm{TS}(5-\mathrm{exo}) \quad \mathrm{HF}=-1070.147916$

02

$\mathrm{C}$

$\begin{array}{llr}\text { C } & 1 & 1.526559\end{array}$

$\begin{array}{lllll}\mathrm{C} & 2 & 1.535233 & 1 & 105.946158\end{array}$

$\begin{array}{lllllll}\mathrm{S} & 1 & 2.634537 & 2 & 138.100474 & 3 & 34.434163\end{array}$

$\begin{array}{lllllll}\mathrm{O} & 4 & 1.473743 & 1 & 89.336615 & 2 & 151.799275\end{array}$

$\begin{array}{lllllll}\mathrm{O} & 4 & 1.473492 & 1 & 100.784068 & 2 & -89.060638\end{array}$

$\begin{array}{lllllll}\text { C } & 3 & 1.515099 & 2 & 110.288980 & 1 & -46.751871\end{array}$

$\begin{array}{lllllll}\mathrm{C} & 7 & 1.387601 & 3 & 120.988820 & 2 & 137.572840\end{array}$

$\begin{array}{lllllll}\mathrm{N} & 1 & 1.468013 & 2 & 104.842617 & 3 & 51.858390\end{array}$

$\begin{array}{lllllll}\mathrm{C} & 4 & 1.792724 & 1 & 131.489501 & 2 & 37.046853\end{array}$

$\begin{array}{lllllll}\mathrm{C} & 10 & 1.397696 & 4 & 119.806151 & 1 & 53.710142\end{array}$

$\begin{array}{lllllll}\text { C } & 11 & 1.391329 & 10 & 119.176973 & 4 & 177.164661\end{array}$

$\begin{array}{lllllll}\text { C } & 12 & 1.404465 & 11 & 121.204440 & 10 & -0.797587\end{array}$

$\begin{array}{lllllll}\text { C } & 13 & 1.399991 & 12 & 118.396216 & 11 & 0.346188\end{array}$

$\begin{array}{lllllll}\text { C } & 10 & 1.393343 & 4 & 119.246613 & 1 & -130.056246\end{array}$ 


$\begin{array}{ccccccc}\mathrm{C} & 13 & 1.510273 & 12 & 120.529126 & 11 & -178.585367 \\ \mathrm{H} & 1 & 1.097062 & 2 & 111.932647 & 3 & 169.234526 \\ \mathrm{H} & 1 & 1.100017 & 2 & 110.633233 & 3 & -70.450573 \\ \mathrm{H} & 2 & 1.096745 & 1 & 109.388248 & 9 & -66.931001 \\ \mathrm{H} & 2 & 1.095404 & 1 & 111.651727 & 9 & 173.794334 \\ \mathrm{H} & 3 & 1.097070 & 2 & 109.859064 & 1 & 73.629946 \\ \mathrm{H} & 3 & 1.097807 & 2 & 110.986899 & 1 & -168.069032 \\ \mathrm{H} & 7 & 1.086686 & 3 & 115.898223 & 2 & -70.558400 \\ \mathrm{H} & 8 & 1.085533 & 7 & 121.085798 & 3 & -20.725742 \\ \mathrm{H} & 8 & 1.084942 & 7 & 121.351342 & 3 & 166.737809 \\ \mathrm{H} & 11 & 1.084818 & 10 & 120.021075 & 4 & -3.235173 \\ \mathrm{H} & 12 & 1.087569 & 11 & 119.364955 & 10 & 179.528015 \\ \mathrm{H} & 14 & 1.087306 & 13 & 119.488495 & 12 & -179.916322 \\ \mathrm{H} & 15 & 1.084799 & 10 & 119.493678 & 4 & 3.087936 \\ \mathrm{H} & 16 & 1.095754 & 13 & 111.456730 & 12 & -42.919375 \\ \mathrm{H} & 16 & 1.097840 & 13 & 110.991095 & 12 & 76.352389 \\ \mathrm{H} & 16 & 1.094396 & 13 & 111.529301 & 12 & -163.841149\end{array}$

$\mathrm{A}(\mathrm{R}=\mathrm{H})$ - $\mathrm{TS}(6$-endo $)$

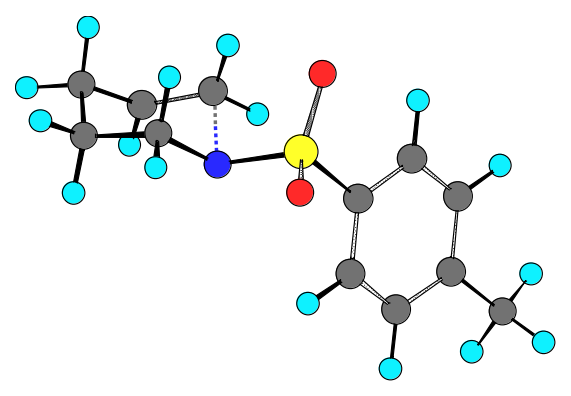

$\% \operatorname{chk}=\mathbf{A}(\mathbf{R}=\mathbf{H})-\mathbf{T S}(6-$ endo $)$

\# ub3lyp/6-31g* opt=(ts,noeigen,readfc,nofreeze) freq=noraman 
calculate for $\mathrm{A}(\mathrm{R}=\mathrm{H})-\mathrm{TS}(6-\mathrm{endo}) \quad \mathrm{HF}=-1070.145060$

02

$\mathrm{C}$

C $\quad 1 \quad 1.496264$

$\begin{array}{lllll}\mathrm{C} & 2 & 1.374053 & 1 & 120.024211\end{array}$

$\begin{array}{lllllll}\mathrm{N} & 3 & 2.147630 & 2 & 96.966989 & 1 & 64.235063\end{array}$

$\begin{array}{lllllll}\mathrm{C} & 4 & 1.473833 & 3 & 101.549847 & 2 & -58.335022\end{array}$

$\begin{array}{lllllll}\text { C } & 5 & 1.532479 & 4 & 111.282697 & 3 & 60.076344\end{array}$

$\begin{array}{lllllll}\mathrm{S} & 4 & 1.694531 & 3 & 106.120518 & 2 & -174.965232\end{array}$

$\begin{array}{lllllll}\mathrm{O} & 7 & 1.475756 & 4 & 112.682845 & 3 & 40.552707\end{array}$

$\begin{array}{lllllll}\mathrm{O} & 7 & 1.473804 & 4 & 106.412930 & 3 & 172.651177\end{array}$

$\begin{array}{lllllll}\text { C } & 7 & 1.791942 & 4 & 100.662422 & 3 & -73.976634\end{array}$

$\begin{array}{lllllll}\text { C } & 10 & 1.393347 & 7 & 119.329207 & 4 & 118.342382\end{array}$

$\begin{array}{lllllll}\text { C } & 11 & 1.395970 & 10 & 119.164102 & 7 & 178.105468\end{array}$

$\begin{array}{lllllll}\mathrm{C} & 12 & 1.399788 & 11 & 121.151490 & 10 & -0.260946\end{array}$

$\begin{array}{lllllll}\mathrm{C} & 13 & 1.404709 & 12 & 118.428205 & 11 & 0.048260\end{array}$

$\begin{array}{lllllll}\text { C } & 14 & 1.390922 & 13 & 121.200811 & 12 & -0.232949\end{array}$

$\begin{array}{lllllll}\mathrm{C} & 13 & 1.510235 & 12 & 121.071068 & 11 & -178.916017\end{array}$

$\begin{array}{lllllll}\mathrm{H} & 2 & 1.088658 & 1 & 118.103814 & 6 & 90.844336\end{array}$

$\begin{array}{lllllll}\mathrm{H} & 1 & 1.098028 & 2 & 110.711052 & 3 & 48.720914\end{array}$

$\begin{array}{lllllll}\mathrm{H} & 1 & 1.095506 & 2 & 111.905840 & 3 & 168.820196\end{array}$

$\begin{array}{lllllll}\mathrm{H} & 6 & 1.096398 & 5 & 109.156309 & 4 & 55.154022\end{array}$

$\begin{array}{lllllll}\mathrm{H} & 6 & 1.097252 & 5 & 108.618793 & 4 & 171.895625\end{array}$

$\begin{array}{lllllll}\mathrm{H} & 5 & 1.101475 & 4 & 112.464610 & 3 & -63.968779\end{array}$

$\begin{array}{lllllll}\mathrm{H} & 5 & 1.098761 & 4 & 106.919178 & 3 & 179.258565\end{array}$

$\begin{array}{lllllll}\mathrm{H} & 3 & 1.085712 & 2 & 120.548826 & 1 & 171.043393\end{array}$

$\begin{array}{lllllll}\mathrm{H} & 3 & 1.086109 & 2 & 120.949541 & 1 & -29.553462\end{array}$

$\begin{array}{lllllll}\mathrm{H} & 11 & 1.084869 & 10 & 119.569005 & 7 & -1.706390\end{array}$ 


$\begin{array}{lllllll}\mathrm{H} & 12 & 1.087278 & 11 & 119.354365 & 10 & 179.914486 \\ \mathrm{H} & 14 & 1.087524 & 13 & 119.428263 & 12 & -179.984637 \\ \mathrm{H} & 15 & 1.084833 & 14 & 120.826415 & 13 & -179.598422 \\ \mathrm{H} & 16 & 1.094370 & 13 & 111.531642 & 12 & -16.833351 \\ \mathrm{H} & 16 & 1.097850 & 13 & 111.018637 & 12 & 103.011047 \\ \mathrm{H} & 16 & 1.095777 & 13 & 111.425146 & 12 & -137.737051\end{array}$

$\mathrm{A}(\mathrm{R}=\mathrm{Me})$-radical

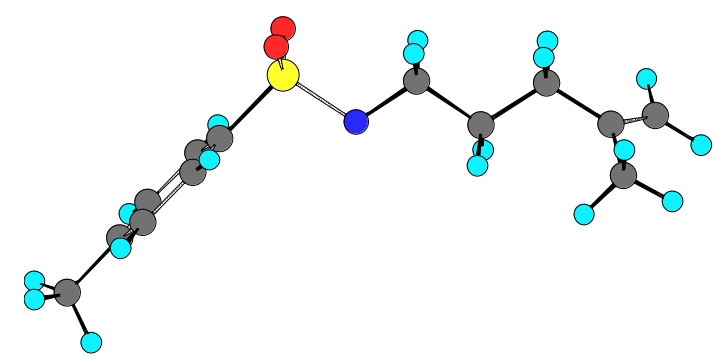

$\%$ chk $=\mathbf{A}(\mathbf{R}=\mathbf{M e})$-radical

\# ub3lyp/6-31g* opt freq=noraman

calculate for $\mathrm{A}(\mathrm{R}=\mathrm{Me})$-radical $\mathrm{HF}=-1109.479262$

02

$\mathrm{C}$

C $\quad 1 \quad 1.529513$

$\begin{array}{lllll}\text { C } & 2 & 1.541398 & 1 & 111.777992\end{array}$

$\begin{array}{lllllll}\mathrm{C} & 3 & 1.515160 & 2 & 113.763439 & 1 & -179.128620\end{array}$

$\begin{array}{lllllll}\mathrm{C} & 4 & 1.336919 & 3 & 121.347967 & 2 & 112.080874\end{array}$

$\begin{array}{lllllll}\mathrm{N} & 1 & 1.453713 & 2 & 110.566222 & 3 & -179.045682\end{array}$

$\begin{array}{lllllll}\mathrm{S} & 6 & 1.703759 & 1 & 112.399142 & 2 & -179.293483\end{array}$

$\begin{array}{lllllll}\mathrm{O} & 7 & 1.471444 & 6 & 107.716820 & 1 & -66.183642\end{array}$

$\begin{array}{lllllll}\mathrm{O} & 7 & 1.471245 & 6 & 107.969876 & 1 & 65.637182\end{array}$ 


$\begin{array}{ccccccc}\mathrm{C} & 7 & 1.784888 & 6 & 101.583979 & 1 & 179.794357 \\ \mathrm{C} & 10 & 1.395282 & 7 & 119.342408 & 6 & 89.222674 \\ \mathrm{C} & 11 & 1.392907 & 10 & 118.958527 & 7 & 179.840072 \\ \mathrm{C} & 12 & 1.402237 & 11 & 121.178367 & 10 & -0.060412 \\ \mathrm{C} & 13 & 1.402553 & 12 & 118.504538 & 11 & -0.572544 \\ \mathrm{C} & 14 & 1.392787 & 13 & 121.183008 & 12 & 0.596225 \\ \mathrm{C} & 13 & 1.509902 & 12 & 120.772937 & 11 & 178.661114 \\ \mathrm{C} & 4 & 1.510151 & 3 & 116.865050 & 2 & -68.301186 \\ \mathrm{H} & 1 & 1.103126 & 2 & 111.065754 & 3 & -57.886802 \\ \mathrm{H} & 1 & 1.102805 & 2 & 111.215344 & 3 & 59.368534 \\ \mathrm{H} & 2 & 1.096409 & 1 & 109.209547 & 6 & 59.409275 \\ \mathrm{H} & 2 & 1.096160 & 1 & 108.598588 & 6 & -56.712266 \\ \mathrm{H} & 3 & 1.097419 & 2 & 109.062122 & 1 & -56.786220 \\ \mathrm{H} & 3 & 1.101281 & 2 & 108.608921 & 1 & 58.920334 \\ \mathrm{H} & 5 & 1.087348 & 4 & 121.773647 & 3 & -179.909670 \\ \mathrm{H} & 5 & 1.087427 & 4 & 121.796964 & 3 & 0.193100 \\ \mathrm{H} & 11 & 1.084760 & 10 & 120.004220 & 7 & 0.867532 \\ \mathrm{H} & 12 & 1.087118 & 11 & 119.334613 & 10 & -179.602187 \\ \mathrm{H} & 14 & 1.087197 & 13 & 119.481891 & 12 & -178.987076 \\ \mathrm{H} & 15 & 1.084753 & 14 & 121.057592 & 13 & -178.976284 \\ \mathrm{H} & 16 & 1.094616 & 13 & 111.505539 & 12 & 26.939466 \\ \mathrm{H} & 16 & 1.094942 & 13 & 111.504309 & 12 & 147.981770 \\ \mathrm{H} & 16 & 1.098106 & 13 & 110.890076 & 12 & -92.600263 \\ \mathrm{H} & 17 & 1.098854 & 4 & 110.949406 & 3 & -57.428255 \\ \mathrm{H} & 17 & 1.097831 & 4 & 111.795934 & 3 & 61.322738 \\ \mathrm{H} & 17 & 1.094047 & 4 & 111.480422 & 3 & -177.954226\end{array}$

$\mathrm{A}(\mathrm{R}=\mathrm{Me})$ - $\mathrm{TS}(5$-exo) 


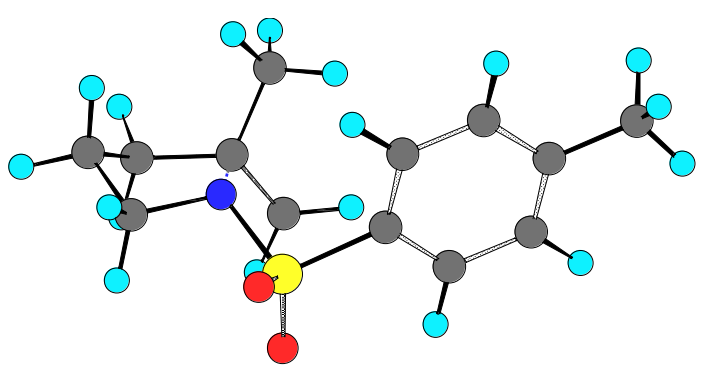

$\% \operatorname{chk}=\mathbf{A}(\mathbf{R}=\mathbf{M e})-\mathbf{T S}(5-\mathbf{e x o})$

\# ub3lyp/6-31g* opt=(ts,noeigen,readfc,nofreeze) freq=noraman

calculate for A $(\mathrm{R}=\mathrm{Me})$ - TS (5-exo) $\mathrm{HF}=-1109.464588$

02

$\mathrm{C}$

C $\quad 1 \quad 1.525213$

$\begin{array}{lllll}\text { C } & 2 & 1.535563 & 1 & 105.784193\end{array}$

$\begin{array}{lllllll}\mathrm{S} & 1 & 2.622129 & 2 & 138.981420 & 3 & 33.596069\end{array}$

$\begin{array}{lllllll}\mathrm{O} & 4 & 1.473782 & 1 & 89.503567 & 2 & 150.299552\end{array}$

$\begin{array}{lllllll}\mathrm{O} & 4 & 1.474053 & 1 & 100.195093 & 2 & -90.696199\end{array}$

$\begin{array}{lllllll}\text { C } & 3 & 1.525056 & 2 & 110.792419 & 1 & -47.010461\end{array}$

$\begin{array}{lllllll}\mathrm{C} & 7 & 1.396626 & 3 & 118.012376 & 2 & 132.929859\end{array}$

$\begin{array}{lllllll}\mathrm{N} & 1 & 1.469622 & 2 & 105.006339 & 3 & 50.534081\end{array}$

$\begin{array}{lllllll}\mathrm{C} & 4 & 1.793337 & 1 & 132.437894 & 2 & 35.322573\end{array}$

$\begin{array}{lllllll}\text { C } & 10 & 1.398284 & 4 & 119.848264 & 1 & 55.626251\end{array}$

$\begin{array}{lllllll}\text { C } & 11 & 1.391341 & 10 & 119.203890 & 4 & 177.305550\end{array}$

$\begin{array}{lllllll}\mathrm{C} & 12 & 1.404964 & 11 & 121.181556 & 10 & -0.771325\end{array}$

$\begin{array}{lllllll}\text { C } & 13 & 1.399643 & 12 & 118.390655 & 11 & -0.150217\end{array}$

$\begin{array}{lllllll}\mathrm{C} & 10 & 1.393063 & 4 & 119.226758 & 1 & -128.376490\end{array}$

$\begin{array}{lllllll}\text { C } & 13 & 1.510299 & 12 & 120.468203 & 11 & 179.558941\end{array}$

$\begin{array}{lllllll}\text { C } & 7 & 1.515757 & 3 & 116.258421 & 2 & -77.801994\end{array}$

$\begin{array}{lllllll}\mathrm{H} & 1 & 1.096981 & 2 & 112.070111 & 3 & 168.393694\end{array}$ 


$\begin{array}{lllllll}\mathrm{H} & 1 & 1.100101 & 2 & 110.441306 & 3 & -71.423391 \\ \mathrm{H} & 2 & 1.096260 & 1 & 109.798838 & 9 & -68.907619 \\ \mathrm{H} & 2 & 1.095423 & 1 & 111.549274 & 9 & 171.951835 \\ \mathrm{H} & 3 & 1.096265 & 2 & 109.345536 & 1 & 73.554115 \\ \mathrm{H} & 3 & 1.097877 & 2 & 111.020837 & 1 & -168.390856 \\ \mathrm{H} & 8 & 1.084636 & 7 & 121.285016 & 3 & -20.568611 \\ \mathrm{H} & 8 & 1.085678 & 7 & 121.063765 & 3 & 167.130552 \\ \mathrm{H} & 11 & 1.084893 & 10 & 119.992441 & 4 & -2.739802 \\ \mathrm{H} & 12 & 1.087618 & 11 & 119.369854 & 10 & 179.784631 \\ \mathrm{H} & 14 & 1.087230 & 13 & 119.475819 & 12 & -179.259467 \\ \mathrm{H} & 15 & 1.084754 & 10 & 119.464247 & 4 & 2.715336 \\ \mathrm{H} & 16 & 1.097688 & 13 & 111.166416 & 12 & -69.906564 \\ \mathrm{H} & 16 & 1.096125 & 13 & 111.338924 & 12 & 49.329717 \\ \mathrm{H} & 16 & 1.094196 & 13 & 111.492016 & 12 & 169.989538 \\ \mathrm{H} & 17 & 1.093006 & 7 & 111.200642 & 3 & 168.699725 \\ \mathrm{H} & 17 & 1.098498 & 7 & 109.440286 & 3 & -71.728066 \\ \mathrm{H} & 17 & 1.092580 & 7 & 111.544478 & 3 & 48.038408\end{array}$

$\mathrm{A}(\mathrm{R}=\mathrm{Me})$-TS (6-endo)

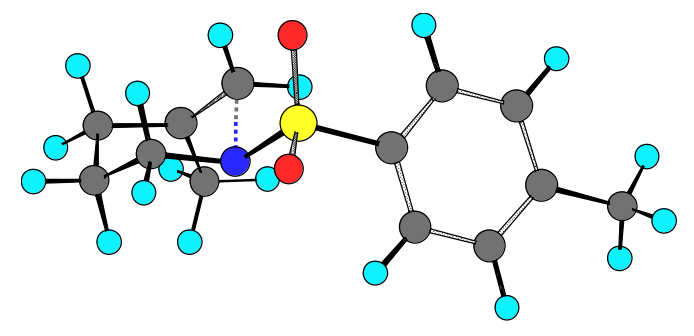

$\%$ chk = A $(\mathbf{R}=\mathbf{M e})$-TS (6-endo $)$

\# ub31yp/6-31g* opt=(ts,noeigen,readfc,nofreeze) freq=noraman

calculate for A $(\mathrm{R}=\mathrm{Me})$-TS (6-endo) $\mathrm{HF}=-1109.466815$ 
02

$\mathrm{C}$

$\begin{array}{lll}\text { C } & 1 & 1.504635\end{array}$

$\begin{array}{lllll}\text { C } & 2 & 1.376817 & 1 & 117.518672\end{array}$

$\begin{array}{lllllll}\mathrm{N} & 3 & 2.151597 & 2 & 96.888902 & 1 & 65.911383\end{array}$

$\begin{array}{lllllll}\mathrm{C} & 4 & 1.473495 & 3 & 101.015119 & 2 & -61.111721\end{array}$

$\begin{array}{lllllll}\mathrm{C} & 5 & 1.532234 & 4 & 110.993278 & 3 & 60.429136\end{array}$

$\begin{array}{lllllll}\mathrm{S} & 4 & 1.690611 & 3 & 106.369336 & 2 & -177.710399\end{array}$

$\begin{array}{lllllll}\mathrm{O} & 7 & 1.476192 & 4 & 112.724710 & 3 & 40.174382\end{array}$

$\begin{array}{lllllll}\mathrm{O} & 7 & 1.474208 & 4 & 106.710195 & 3 & 172.415619\end{array}$

$\begin{array}{lllllll}\mathrm{C} & 7 & 1.792736 & 4 & 100.687171 & 3 & -74.317696\end{array}$

$\begin{array}{lllllll}\text { C } & 10 & 1.393367 & 7 & 119.427634 & 4 & 116.299615\end{array}$

$\begin{array}{lllllll}\mathrm{C} & 11 & 1.395999 & 10 & 119.191254 & 7 & 178.005016\end{array}$

$\begin{array}{lllllll}\text { C } & 12 & 1.399910 & 11 & 121.135633 & 10 & -0.280091\end{array}$

$\begin{array}{lllllll}\text { C } & 13 & 1.404532 & 12 & 118.425147 & 11 & 0.081587\end{array}$

$\begin{array}{lllllll}\text { C } & 14 & 1.391177 & 13 & 121.198328 & 12 & -0.243272\end{array}$

$\begin{array}{lllllll}\mathrm{C} & 13 & 1.510356 & 12 & 121.035993 & 11 & -178.722017\end{array}$

$\begin{array}{lllllll}\mathrm{C} & 2 & 1.499031 & 1 & 118.392213 & 6 & 93.553651\end{array}$

$\begin{array}{lllllll}\mathrm{H} & 1 & 1.097664 & 2 & 110.621933 & 3 & 48.245175\end{array}$

$\begin{array}{lllllll}\mathrm{H} & 1 & 1.096413 & 2 & 111.669132 & 3 & 167.970723\end{array}$

$\begin{array}{lllllll}\mathrm{H} & 6 & 1.096735 & 5 & 109.049505 & 4 & 56.048990\end{array}$

$\begin{array}{lllllll}\mathrm{H} & 6 & 1.097109 & 5 & 108.632419 & 4 & 172.542800\end{array}$

$\begin{array}{lllllll}\mathrm{H} & 5 & 1.101719 & 4 & 112.435279 & 3 & -63.302144\end{array}$

$\begin{array}{lllllll}\mathrm{H} & 5 & 1.098672 & 4 & 107.264254 & 3 & 179.732611\end{array}$

$\begin{array}{lllllll}\mathrm{H} & 3 & 1.086139 & 2 & 120.381918 & 1 & 172.700954\end{array}$

$\begin{array}{lllllll}\mathrm{H} & 3 & 1.085260 & 2 & 121.117664 & 1 & -27.781687\end{array}$

$\begin{array}{lllllll}\mathrm{H} & 11 & 1.084905 & 10 & 119.520563 & 7 & -1.687978\end{array}$

$\begin{array}{lllllll}\mathrm{H} & 12 & 1.087336 & 11 & 119.367753 & 10 & 179.954606\end{array}$ 


$\begin{array}{lllllll}\mathrm{H} & 14 & 1.087552 & 13 & 119.418049 & 12 & -179.936835 \\ \mathrm{H} & 15 & 1.084870 & 14 & 120.847360 & 13 & -179.423107 \\ \mathrm{H} & 16 & 1.094475 & 13 & 111.530275 & 12 & -19.088930 \\ \mathrm{H} & 16 & 1.097942 & 13 & 110.998430 & 12 & 100.680211 \\ \mathrm{H} & 16 & 1.095640 & 13 & 111.457559 & 12 & -140.035714 \\ \mathrm{H} & 17 & 1.093838 & 2 & 112.101583 & 1 & -173.830759 \\ \mathrm{H} & 17 & 1.100324 & 2 & 110.935335 & 1 & -53.035194 \\ \mathrm{H} & 17 & 1.098934 & 2 & 110.834072 & 1 & 64.934087\end{array}$

$\mathrm{A}(\mathrm{R}=\mathrm{Cl})$-radical

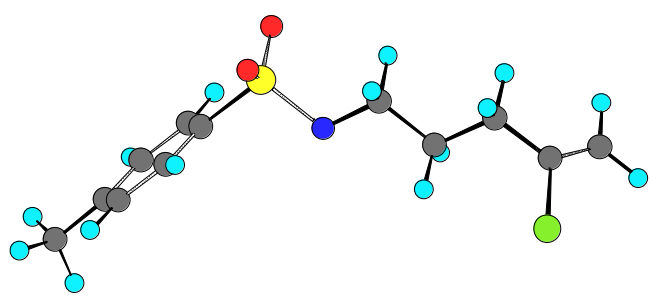

\%chk=A ( $\mathbf{R}=\mathbf{C l})$-radical

\# ub3lyp/6-31g* opt freq=noraman

calculate for A $(\mathrm{R}=\mathrm{Cl})$-radical $\mathrm{HF}=-1529.759272$

02

$\mathrm{C}$

$\begin{array}{llr}\text { C } & 1 & 1.529728\end{array}$

$\begin{array}{lllll}\text { C } & 2 & 1.539800 & 1 & 111.489882\end{array}$

$\begin{array}{lllllll}\mathrm{C} & 3 & 1.504098 & 2 & 113.866643 & 1 & 179.897041\end{array}$

$\begin{array}{lllllll}\mathrm{C} & 4 & 1.330892 & 3 & 126.272591 & 2 & 113.017371\end{array}$

$\begin{array}{lllllll}\mathrm{N} & 1 & 1.453294 & 2 & 110.305333 & 3 & -179.466433\end{array}$

$\begin{array}{lllllll}\mathrm{S} & 6 & 1.704438 & 1 & 112.315527 & 2 & -177.136909\end{array}$ 


$\begin{array}{ccccccc}\mathrm{O} & 7 & 1.470958 & 6 & 108.119393 & 1 & -63.604226 \\ \mathrm{O} & 7 & 1.471515 & 6 & 107.377043 & 1 & 68.082451 \\ \mathrm{C} & 7 & 1.784114 & 6 & 101.587163 & 1 & -177.883652 \\ \mathrm{C} & 10 & 1.395160 & 7 & 119.371371 & 6 & 91.010081 \\ \mathrm{C} & 11 & 1.393112 & 10 & 118.929787 & 7 & 179.892417 \\ \mathrm{C} & 12 & 1.402098 & 11 & 121.185801 & 10 & -0.054875 \\ \mathrm{C} & 13 & 1.402782 & 12 & 118.511584 & 11 & -0.583635 \\ \mathrm{C} & 14 & 1.392464 & 13 & 121.179497 & 12 & 0.604841 \\ \mathrm{C} & 13 & 1.509798 & 12 & 120.808981 & 11 & 178.623516 \\ \mathrm{Cl} & 4 & 1.774270 & 3 & 114.367983 & 2 & -66.970802 \\ \mathrm{H} & 1 & 1.103073 & 2 & 111.082235 & 3 & -58.702719 \\ \mathrm{H} & 1 & 1.102758 & 2 & 111.347666 & 3 & 58.761927 \\ \mathrm{H} & 2 & 1.096049 & 1 & 109.330754 & 6 & 59.045552 \\ \mathrm{H} & 2 & 1.094911 & 1 & 109.126460 & 6 & -57.700732 \\ \mathrm{H} & 3 & 1.097467 & 2 & 109.740154 & 1 & -59.461490 \\ \mathrm{H} & 3 & 1.098698 & 2 & 109.422465 & 1 & 57.564733 \\ \mathrm{H} & 5 & 1.084030 & 4 & 122.519157 & 3 & -179.858130 \\ \mathrm{H} & 5 & 1.086347 & 4 & 119.693365 & 3 & 0.278484 \\ \mathrm{H} & 11 & 1.084749 & 10 & 120.027807 & 7 & 0.854089 \\ \mathrm{H} & 12 & 1.087069 & 11 & 119.328194 & 10 & -179.641653 \\ \mathrm{H} & 14 & 1.087176 & 13 & 119.487487 & 12 & -178.978033 \\ \mathrm{H} & 15 & 1.084734 & 14 & 121.056737 & 13 & -178.981484 \\ \mathrm{H} & 16 & 1.094566 & 13 & 111.524340 & 12 & 26.179415 \\ \mathrm{H} & 16 & 1.094978 & 13 & 111.501194 & 12 & 147.227252 \\ & 16 & 1.098073 & 13 & 110.868417 & 12 & -93.381540\end{array}$

$\mathrm{A}(\mathrm{R}=\mathrm{Cl})$-TS (5-exo) 


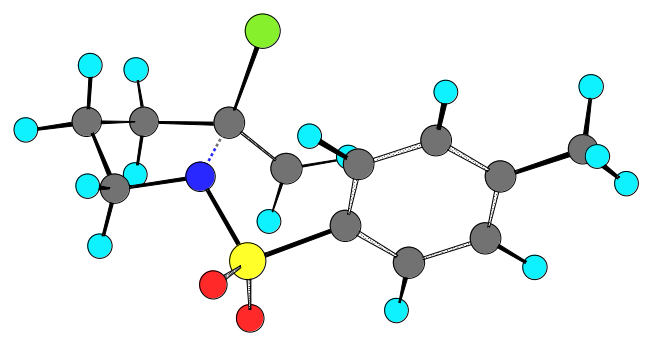

$\% \operatorname{chk}=\mathbf{A}(\mathbf{R}=\mathbf{C l})-\mathbf{T S}(\mathbf{5}-\mathbf{e x o})$

\# ub3lyp/6-31g* opt=(ts,noeigen,readfc,nofreeze) freq=noraman

calculate for $\mathrm{A}(\mathrm{R}=\mathrm{Cl})$ - TS $(5$-exo $) \quad \mathrm{HF}=-1529.739485$

02

C

C $\quad 1 \quad 1.526978$

C $\quad 2 \quad 1.530979 \quad 1 \quad 105.255828$

$\begin{array}{lllllll}\mathrm{S} & 1 & 2.628721 & 2 & 137.876333 & 3 & 29.089174\end{array}$

$\begin{array}{lllllll}\mathrm{O} & 4 & 1.472428 & 1 & 88.940125 & 2 & 156.405997\end{array}$

$\begin{array}{lllllll}\mathrm{O} & 4 & 1.473987 & 1 & 99.571486 & 2 & -84.578469\end{array}$

$\begin{array}{lllllll}\text { C } & 3 & 1.517749 & 2 & 110.439870 & 1 & -49.559284\end{array}$

$\begin{array}{lllllll}\mathrm{C} & 7 & 1.388995 & 3 & 121.584845 & 2 & 140.011038\end{array}$

$\begin{array}{lllllll}\mathrm{N} & 1 & 1.469710 & 2 & 105.153052 & 3 & 49.419485\end{array}$

$\begin{array}{lllllll}\mathrm{C} & 4 & 1.789572 & 1 & 132.385706 & 2 & 41.180783\end{array}$

$\begin{array}{lllllll}\mathrm{C} & 10 & 1.398116 & 4 & 119.857541 & 1 & 51.459126\end{array}$

$\begin{array}{lllllll}\mathrm{C} & 11 & 1.390609 & 10 & 119.080213 & 4 & 176.817977\end{array}$

$\begin{array}{lllllll}\mathrm{C} & 12 & 1.404796 & 11 & 121.224624 & 10 & -0.697209\end{array}$

$\begin{array}{lllllll}\mathrm{C} & 13 & 1.399749 & 12 & 118.435172 & 11 & 0.046390\end{array}$

$\begin{array}{lllllll}\text { C } & 10 & 1.393430 & 4 & 119.043378 & 1 & -132.756560\end{array}$

$\begin{array}{lllllll}\text { C } & 13 & 1.510104 & 12 & 120.376047 & 11 & -179.828955\end{array}$

$\begin{array}{lllllll}\mathrm{Cl} & 7 & 1.783814 & 3 & 113.710251 & 2 & -75.029119\end{array}$

$\begin{array}{lllllll}\mathrm{H} & 1 & 1.096685 & 2 & 112.059321 & 3 & 166.826329\end{array}$ 


$\begin{array}{ccccccc}\mathrm{H} & 1 & 1.099754 & 2 & 110.410574 & 3 & -72.802506 \\ \mathrm{H} & 2 & 1.095149 & 1 & 110.186104 & 9 & -69.358439 \\ \mathrm{H} & 2 & 1.094866 & 1 & 111.556844 & 9 & 170.560151 \\ \mathrm{H} & 3 & 1.097044 & 2 & 109.753965 & 1 & 68.785567 \\ \mathrm{H} & 3 & 1.095320 & 2 & 112.160425 & 1 & -171.533173 \\ \mathrm{H} & 8 & 1.084267 & 7 & 119.323907 & 3 & -26.866509 \\ \mathrm{H} & 8 & 1.082844 & 7 & 121.600284 & 3 & 162.133224 \\ \mathrm{H} & 11 & 1.084460 & 10 & 120.104212 & 4 & -3.896362 \\ \mathrm{H} & 12 & 1.087441 & 11 & 119.307598 & 10 & 179.461214 \\ \mathrm{H} & 14 & 1.087126 & 13 & 119.502719 & 12 & -179.652546 \\ \mathrm{H} & 15 & 1.084748 & 10 & 119.619001 & 4 & 3.387026 \\ \mathrm{H} & 16 & 1.096900 & 13 & 111.222209 & 12 & -59.384370 \\ \mathrm{H} & 16 & 1.096872 & 13 & 111.179456 & 12 & 59.765373 \\ \mathrm{H} & 16 & 1.094126 & 13 & 111.548504 & 12 & -179.852534\end{array}$

$\mathrm{A}(\mathrm{R}=\mathrm{Cl})$ - $\mathrm{TS}$ (6-endo)

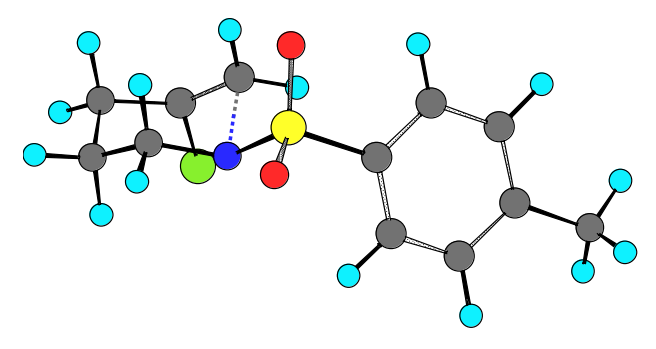

$\% \operatorname{chk}=\mathbf{A}(\mathbf{R}=\mathbf{C l})$-TS (6-endo)

\# ub3lyp/6-31g* opt=(ts,noeigen,readfc,nofreeze) freq=noraman

calculate for $\mathrm{A}(\mathrm{R}=\mathrm{Cl})$ - $\mathrm{TS}(6-\mathrm{endo}) \quad \mathrm{HF}=-1529.745322$

02

$\mathrm{C}$ 


$\begin{array}{lllllll}\mathrm{C} & 1 & 1.497661 & & & & \\ \mathrm{C} & 2 & 1.369642 & 1 & 121.051102 & & \\ \mathrm{~N} & 3 & 2.163495 & 2 & 97.066080 & 1 & 62.613091 \\ \mathrm{C} & 4 & 1.473427 & 3 & 101.862160 & 2 & -56.952330 \\ \mathrm{C} & 5 & 1.532797 & 4 & 111.373198 & 3 & 59.563257 \\ \mathrm{~S} & 4 & 1.696690 & 3 & 105.855333 & 2 & -173.437922 \\ \mathrm{O} & 7 & 1.475905 & 4 & 112.191323 & 3 & 40.660433 \\ \mathrm{O} & 7 & 1.473096 & 4 & 106.400779 & 3 & 172.571077 \\ \mathrm{C} & 7 & 1.790411 & 4 & 100.726509 & 3 & -73.885328 \\ \mathrm{C} & 10 & 1.393413 & 7 & 119.376119 & 4 & 117.360033 \\ \mathrm{C} & 11 & 1.395941 & 10 & 119.125522 & 7 & 178.196453 \\ \mathrm{C} & 12 & 1.399758 & 11 & 121.149649 & 10 & -0.221229 \\ \mathrm{C} & 13 & 1.404824 & 12 & 118.441950 & 11 & 0.018174 \\ \mathrm{C} & 14 & 1.390634 & 13 & 121.211662 & 12 & -0.201632 \\ \mathrm{C} & 13 & 1.510100 & 12 & 121.082369 & 11 & -179.048341 \\ \mathrm{Cl} & 2 & 1.750032 & 1 & 116.576813 & 6 & 90.930560 \\ \mathrm{H} & 1 & 1.097583 & 2 & 108.989585 & 3 & 49.632398 \\ \mathrm{H} & 1 & 1.093490 & 2 & 111.639980 & 3 & 168.688671 \\ \mathrm{H} & 6 & 1.095838 & 5 & 109.473305 & 4 & 55.093888 \\ \mathrm{H} & 6 & 1.096780 & 5 & 108.590316 & 4 & 172.128422 \\ \mathrm{H} & 5 & 1.101726 & 4 & 112.403867 & 3 & -64.540742 \\ \mathrm{H} & 5 & 1.098783 & 4 & 106.967119 & 3 & 178.814339 \\ \mathrm{H} & 3 & 1.083955 & 2 & 121.048213 & 1 & 169.645993 \\ \mathrm{H} & 3 & 1.085297 & 2 & 119.155513 & 1 & -31.354008 \\ \mathrm{H} & 11 & 1.084858 & 10 & 119.626099 & 7 & -1.526833 \\ \mathrm{H} & 12 & 1.087188 & 11 & 119.345038 & 10 & 179.952480 \\ \mathrm{H} & 1.087424 & 13 & 119.434918 & 12 & 179.970759 \\ \mathrm{H} & 1.084791 & 14 & 120.812287 & 13 & -179.633305 \\ \mathrm{H} & 1.094262 & 13 & 111.522987 & 12 & -15.114020\end{array}$




$\begin{array}{lrrrrrr}\mathrm{H} & 16 & 1.097753 & 13 & 111.014349 & 12 & 104.792446 \\ \mathrm{H} & 16 & 1.095828 & 13 & 111.390754 & 12 & -135.974351\end{array}$




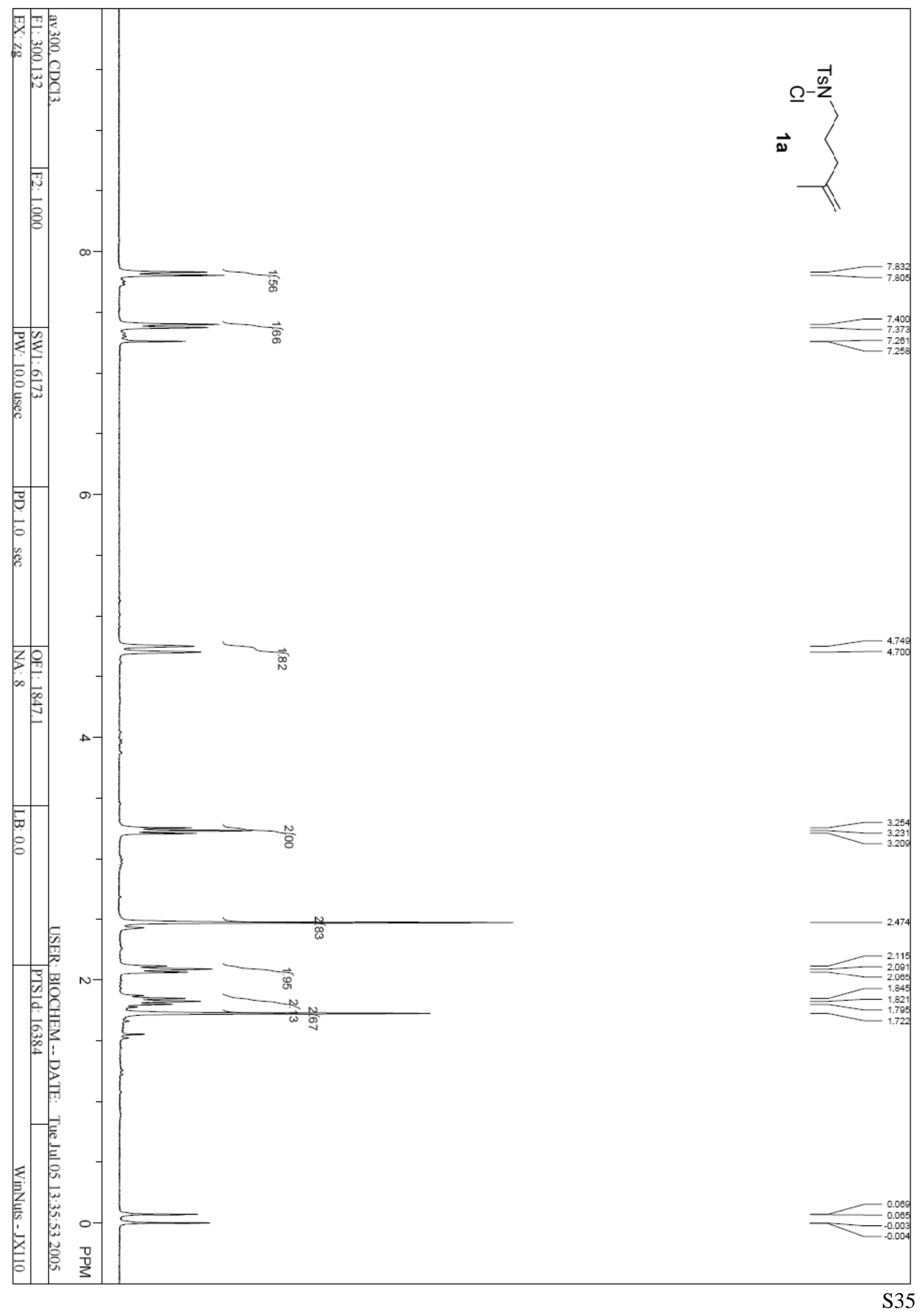




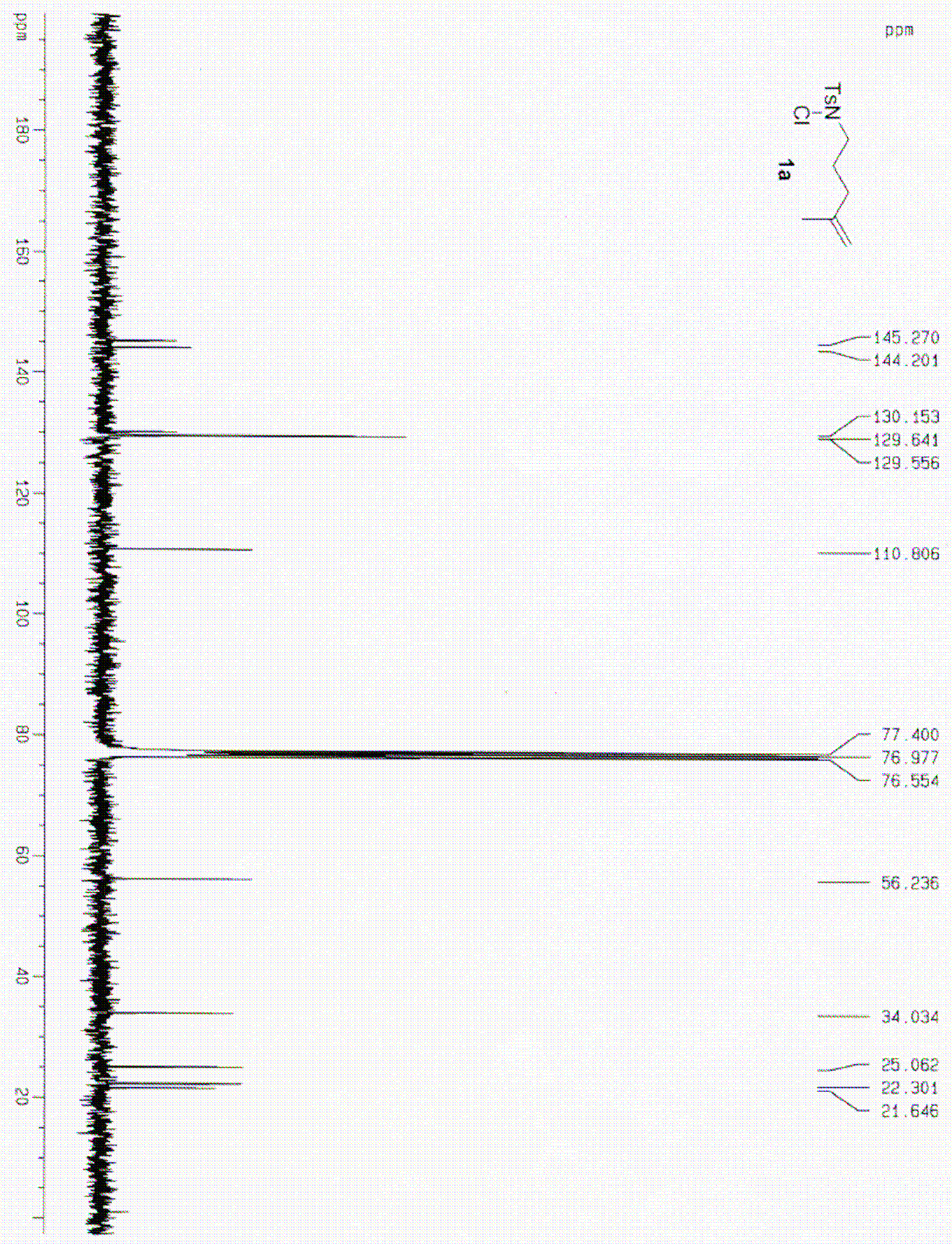




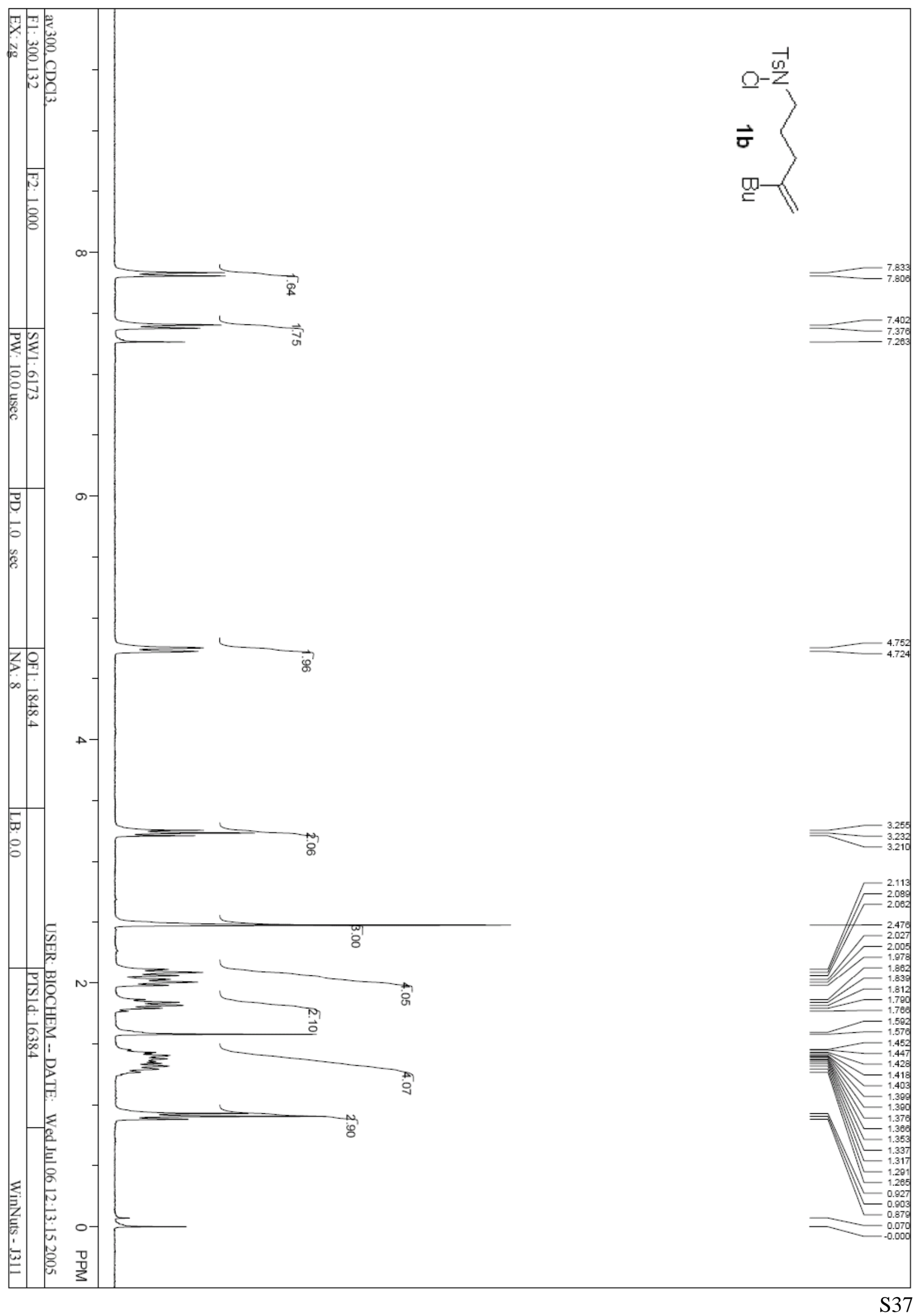




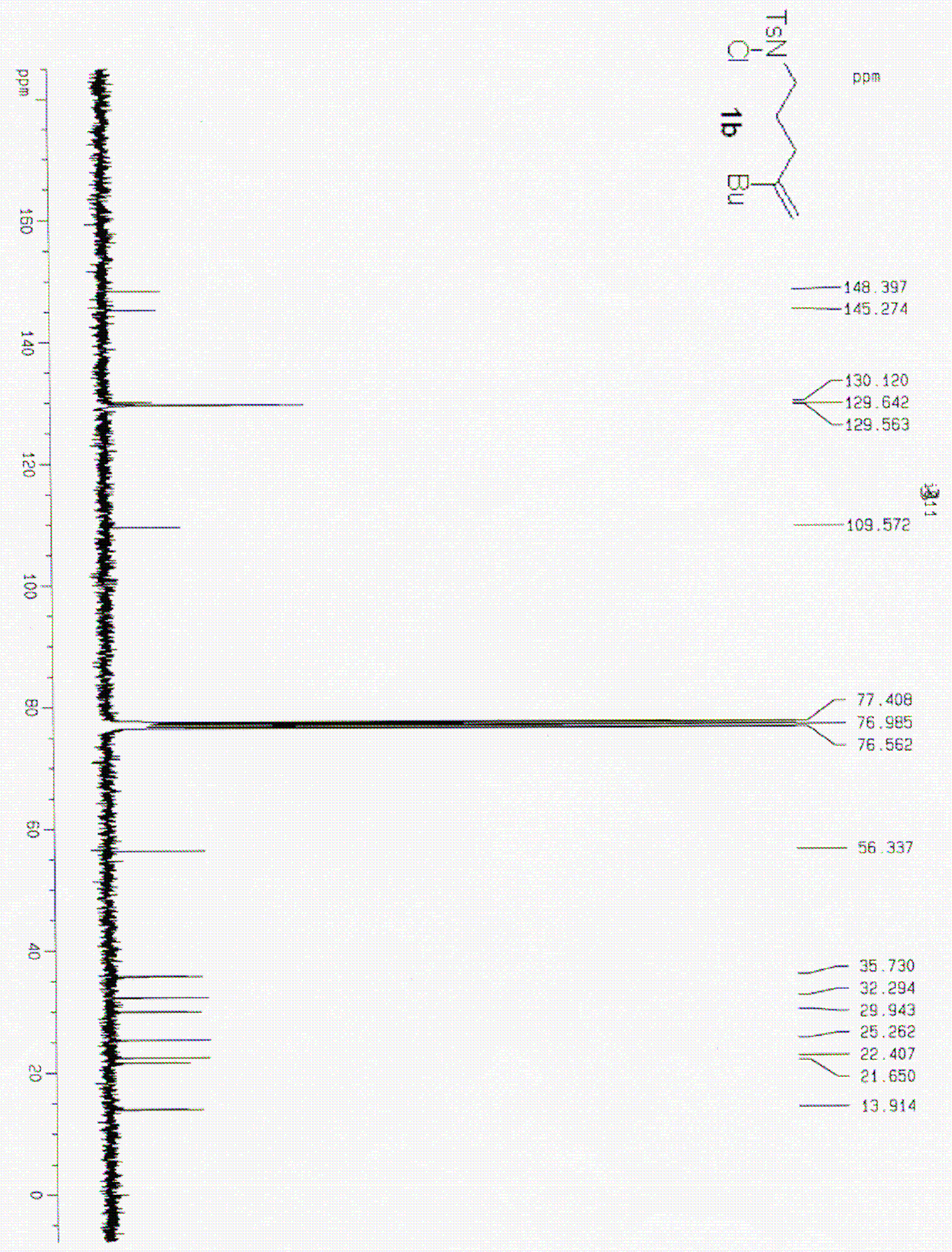

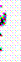




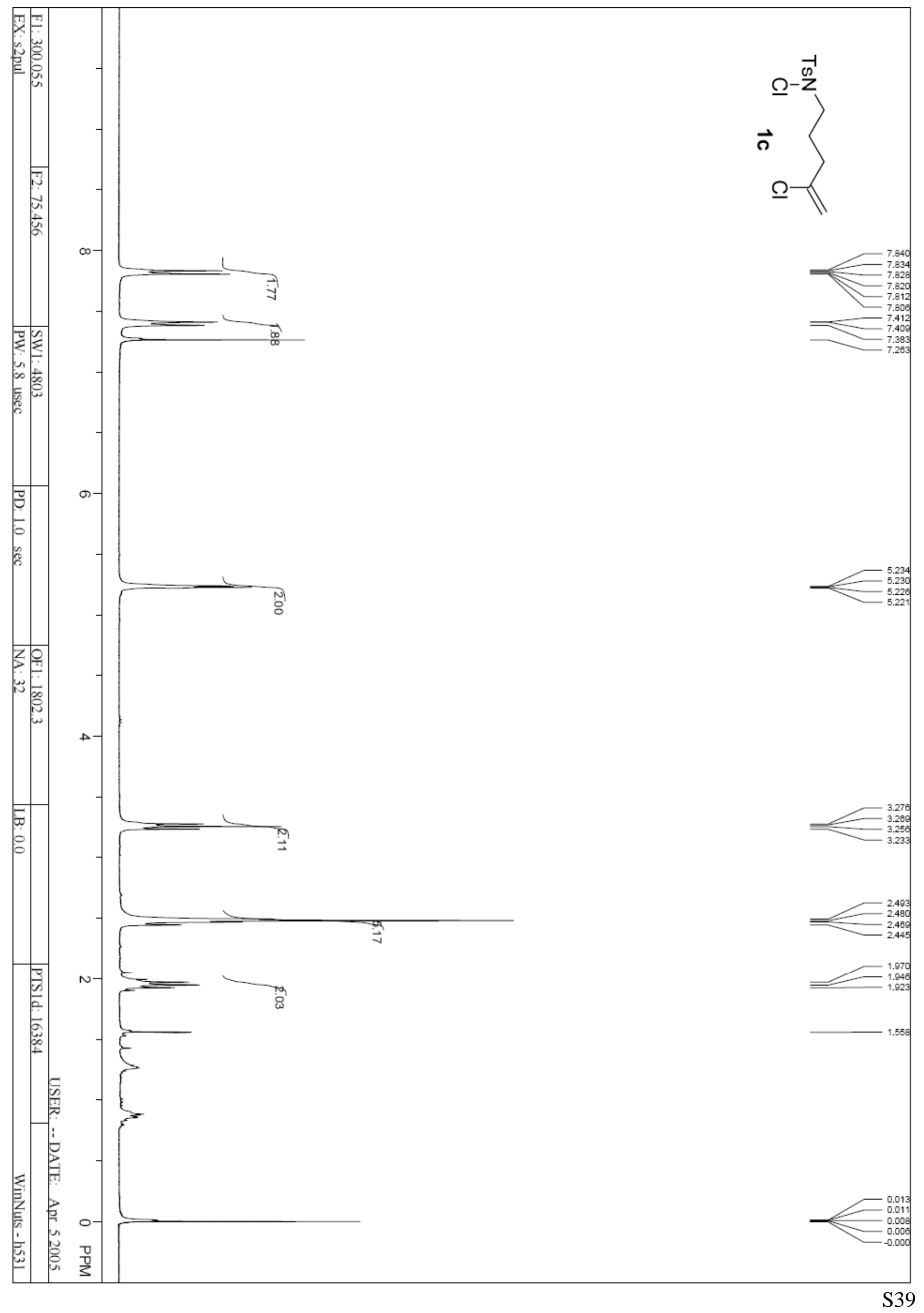




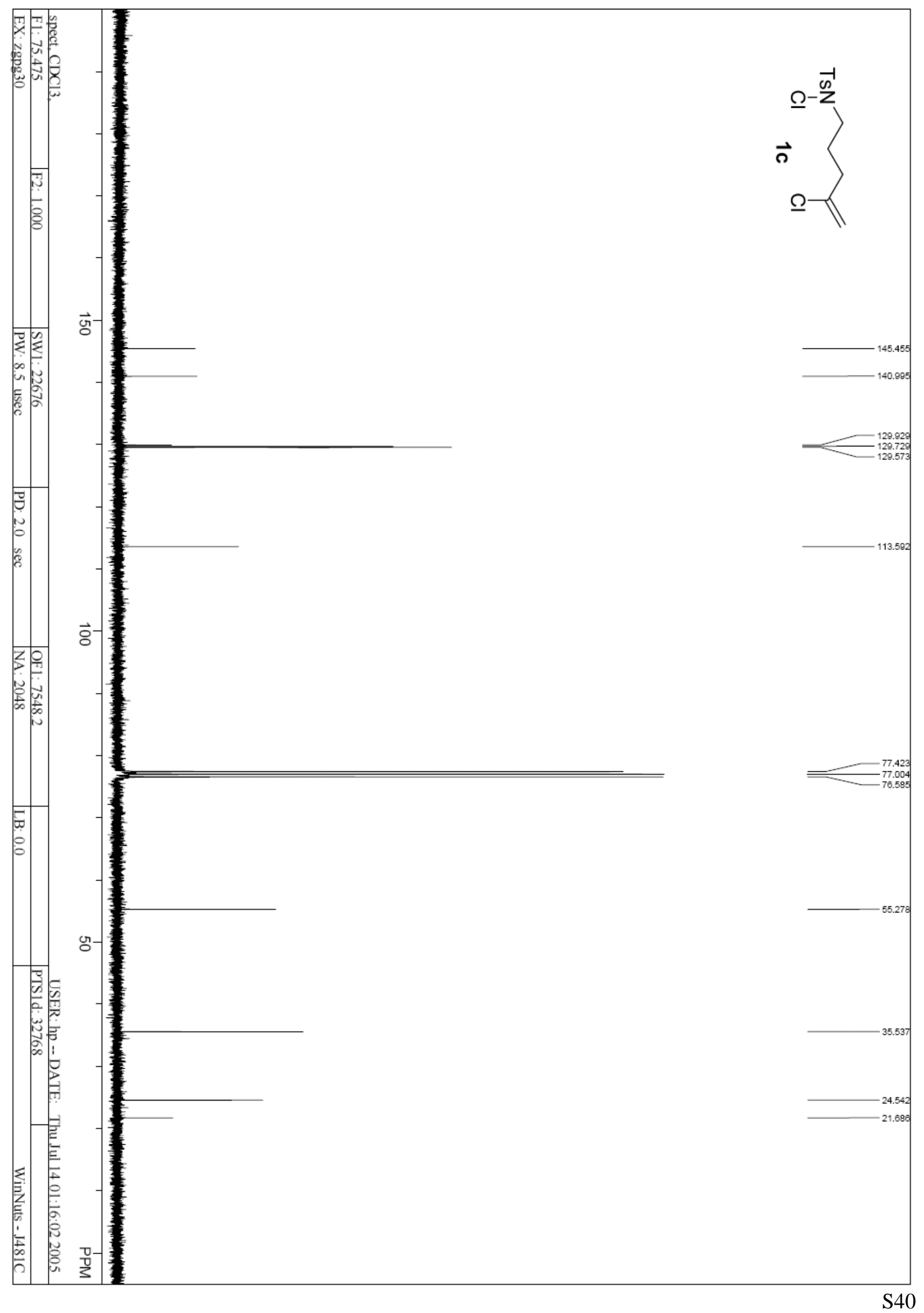




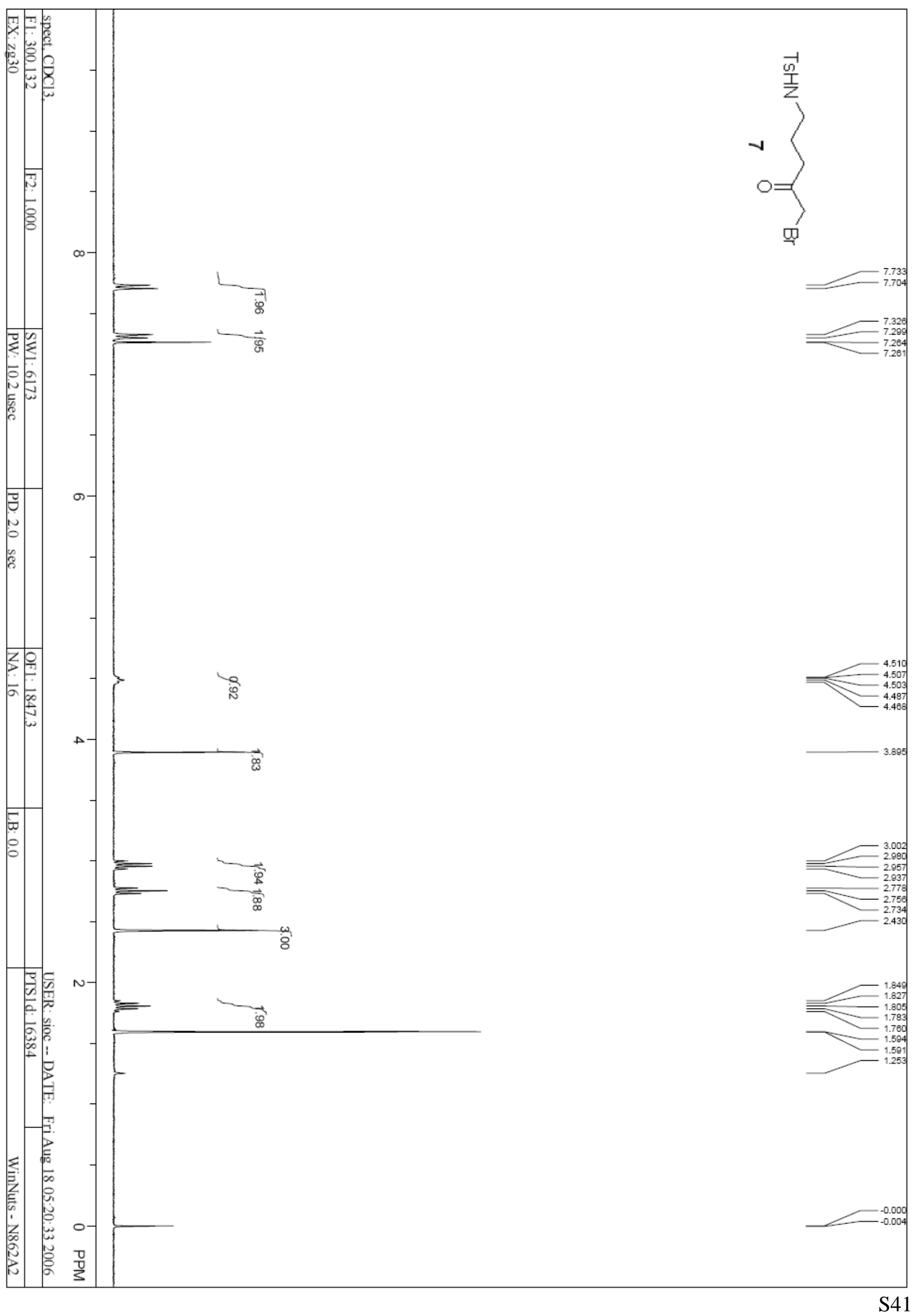




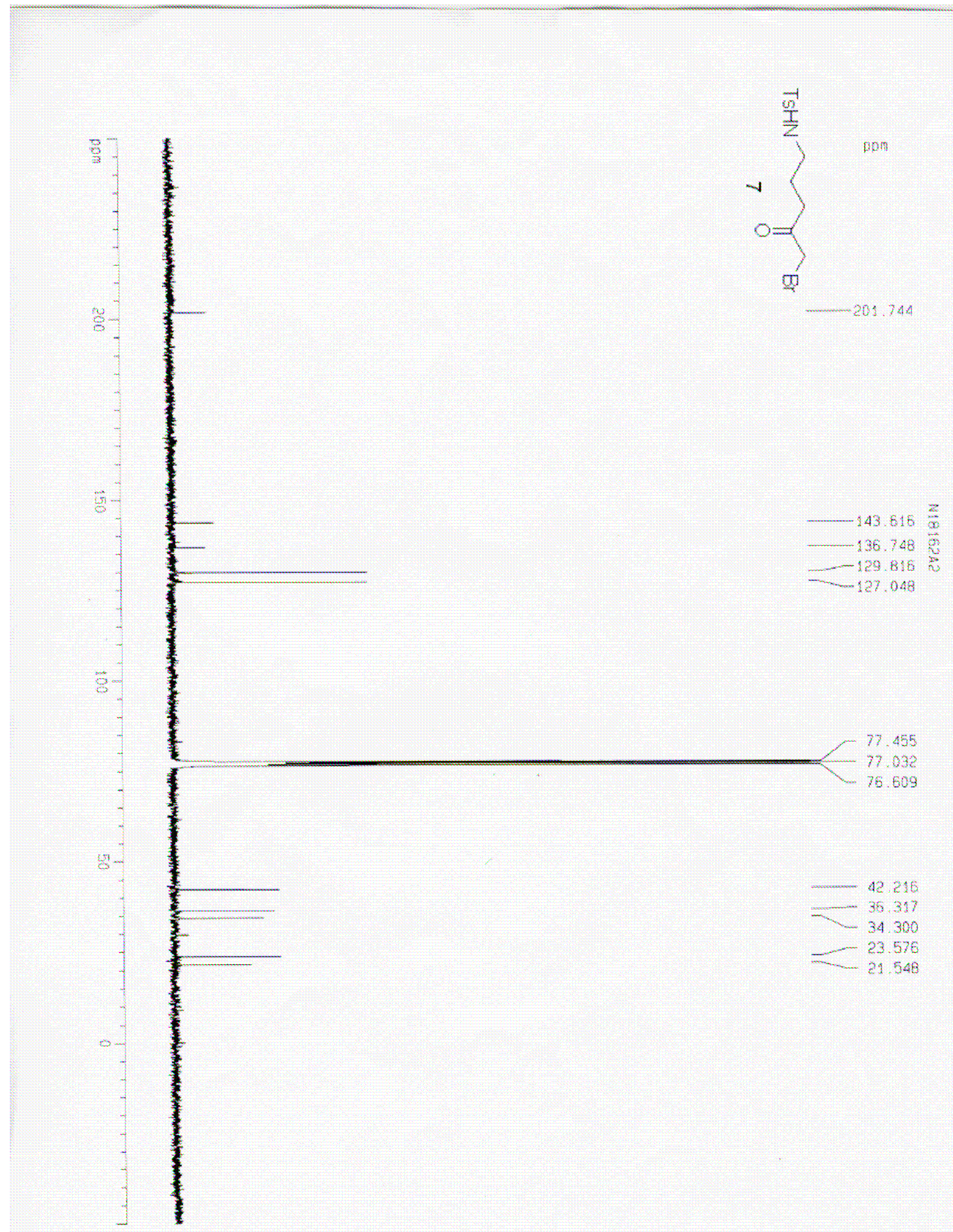




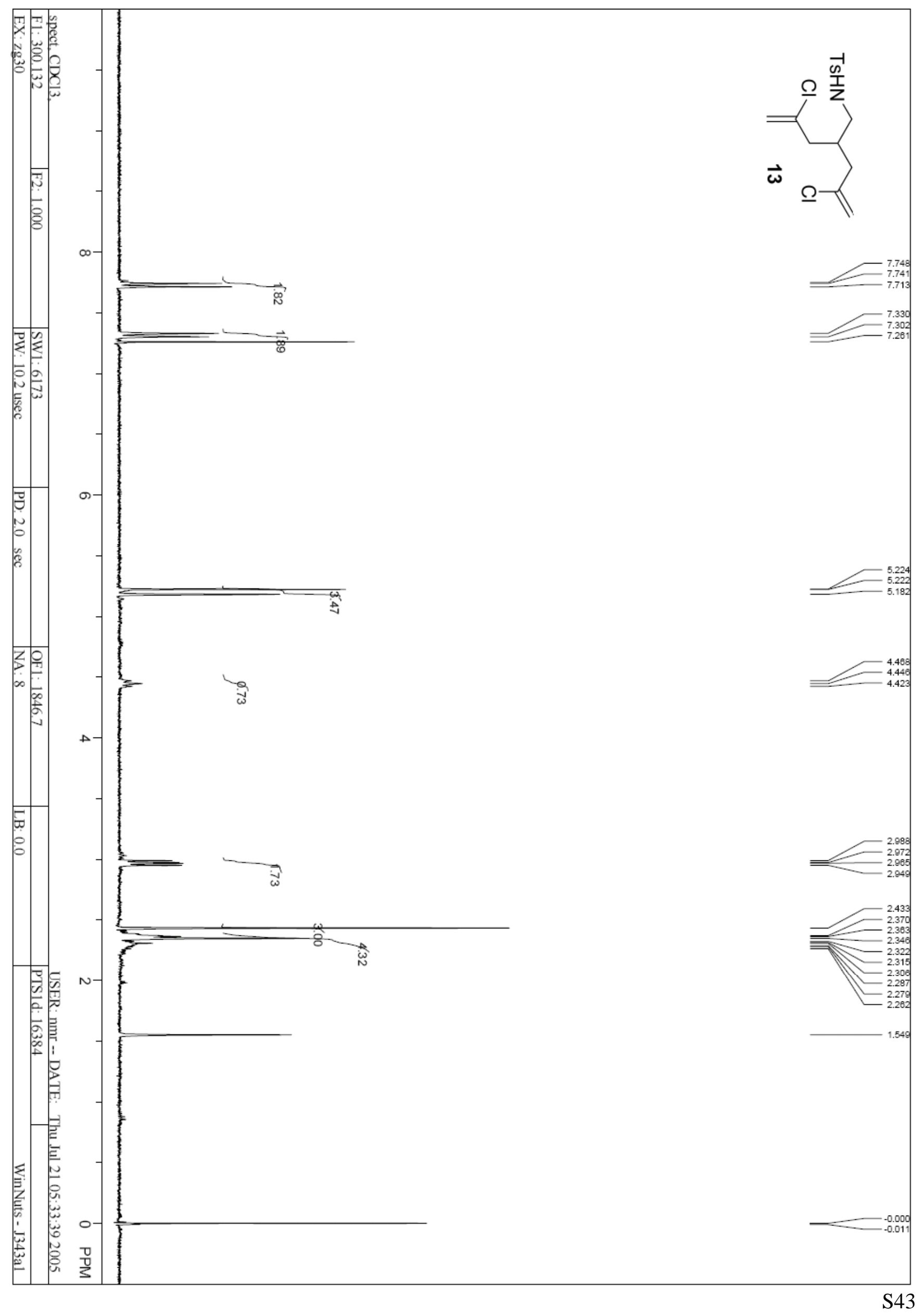




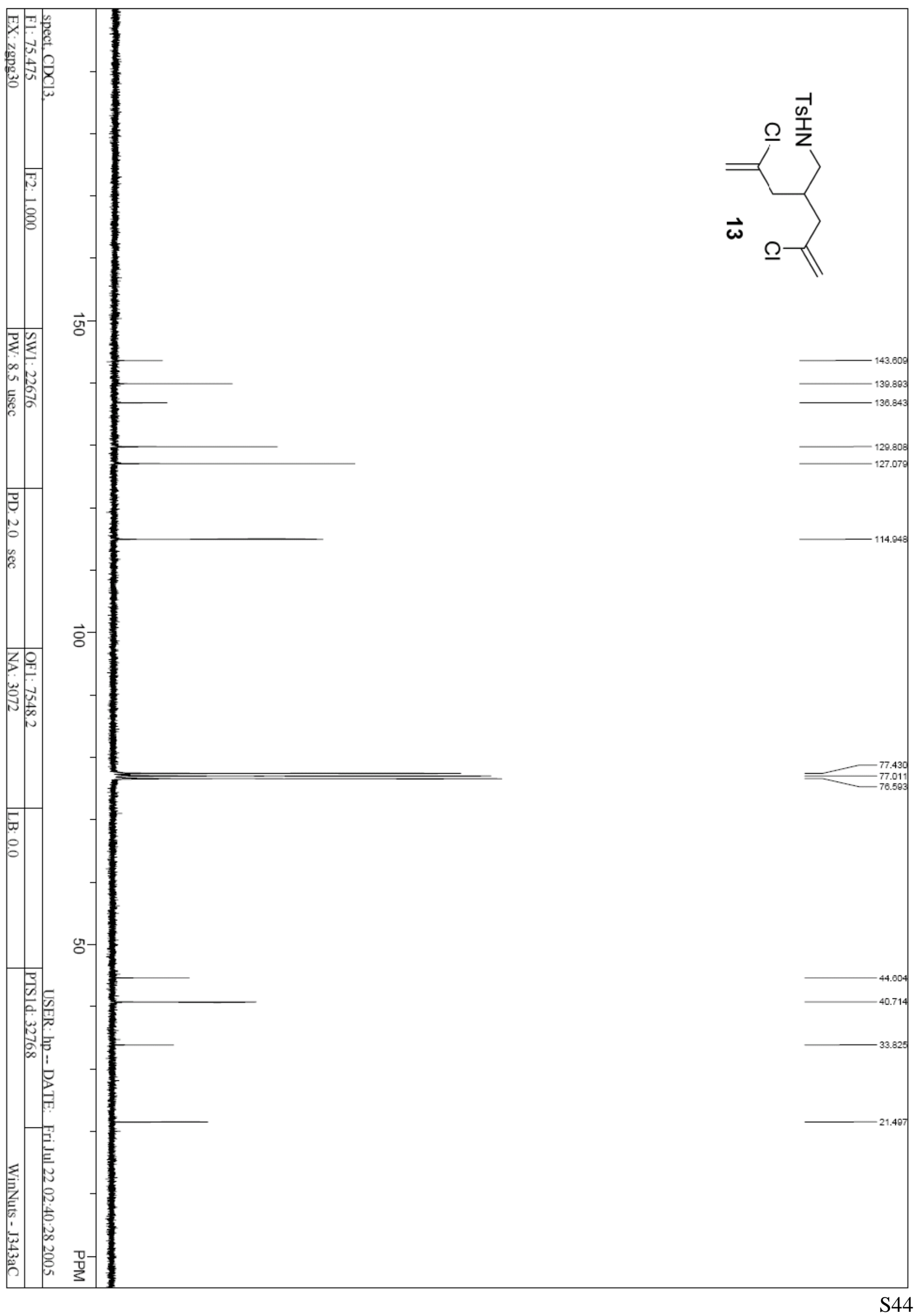




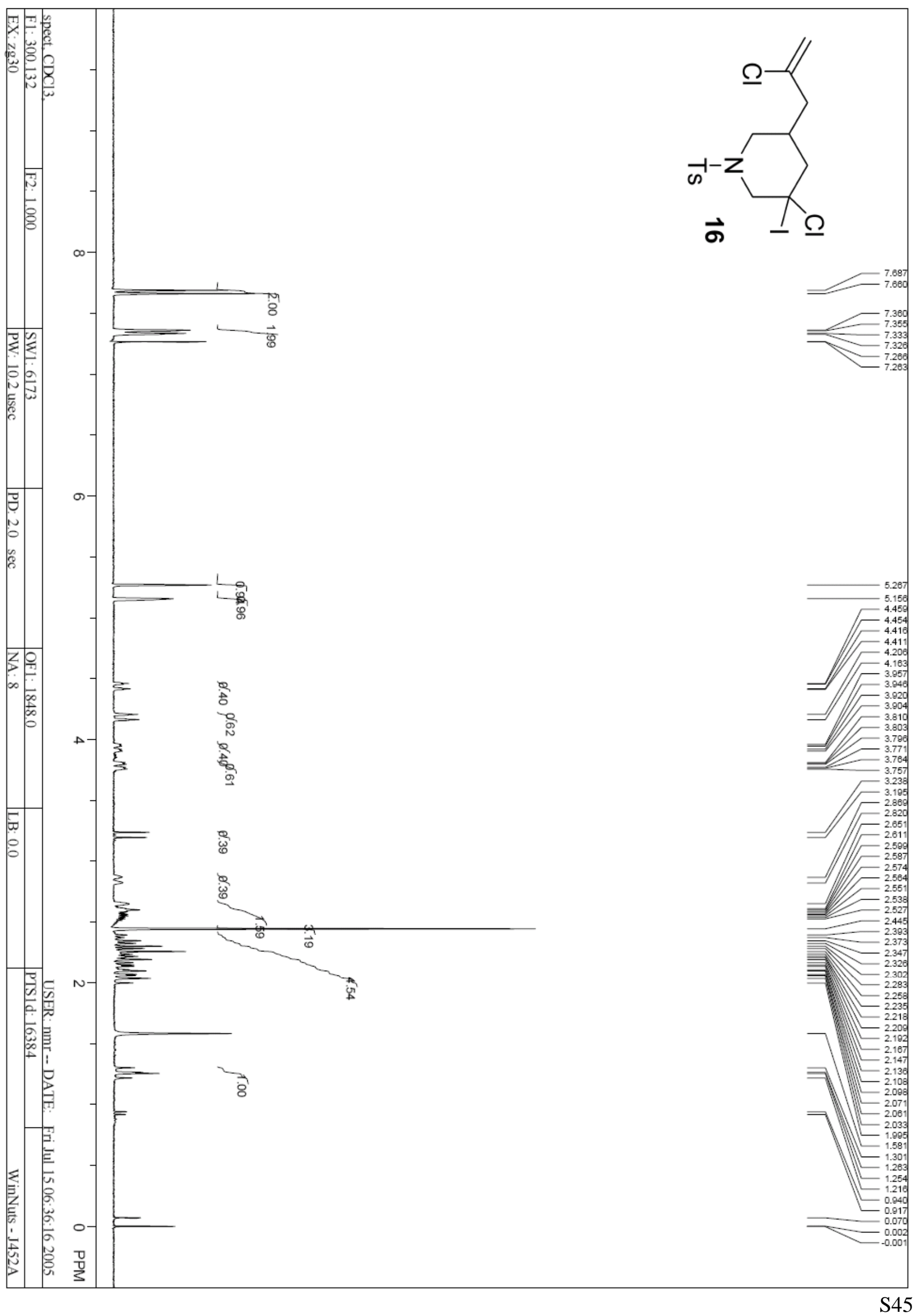



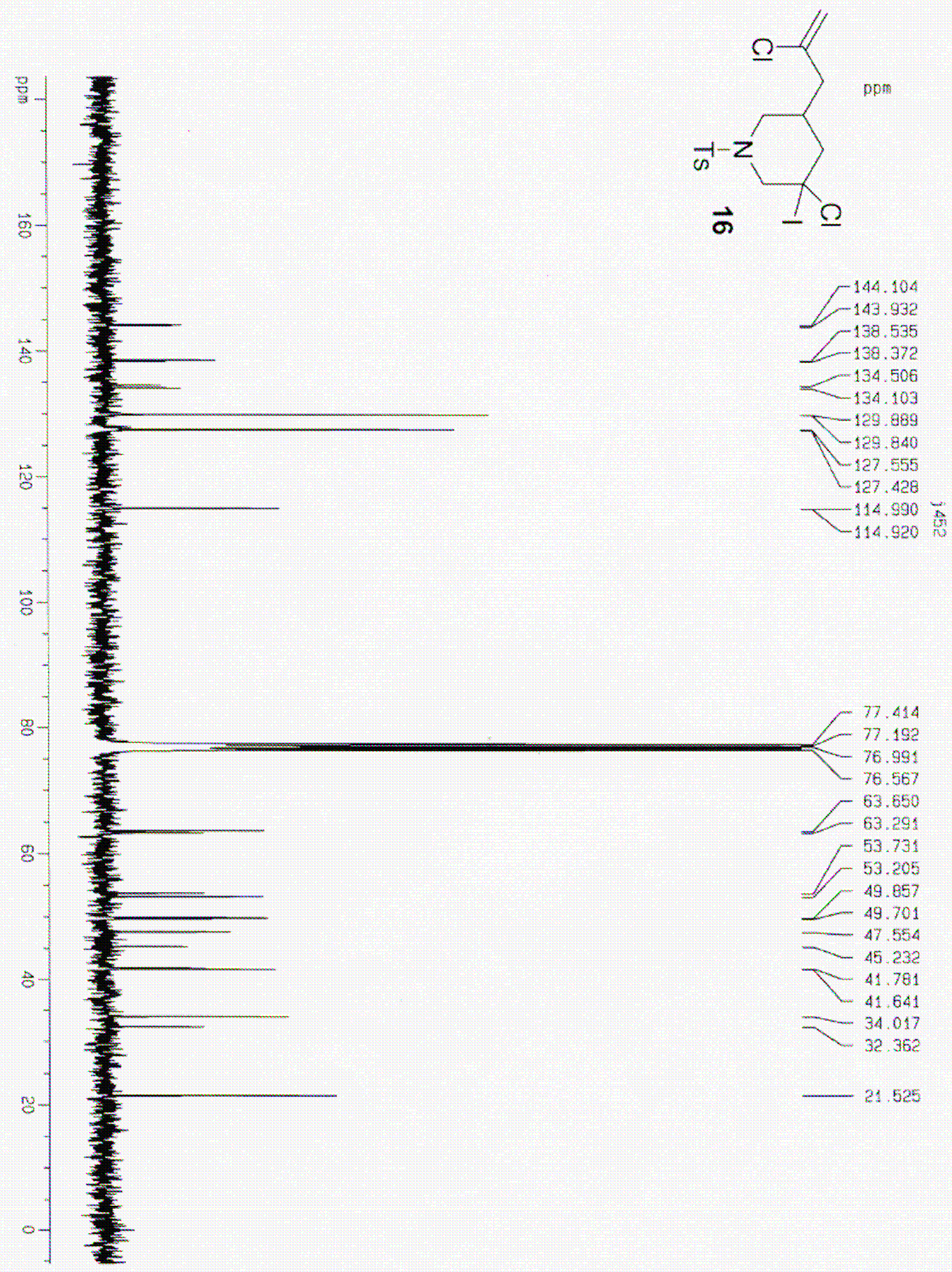

$-77.193$

$-76.99$

$-76.567$

- 63.550

- $63.29 \%$

- 53.731

$-53.205$

49.657

$-49.703$

$-047556$

$\longrightarrow 45232$

Xu 41.781

$-41.641$

$=31.017$

$\times 32352$

21.525 


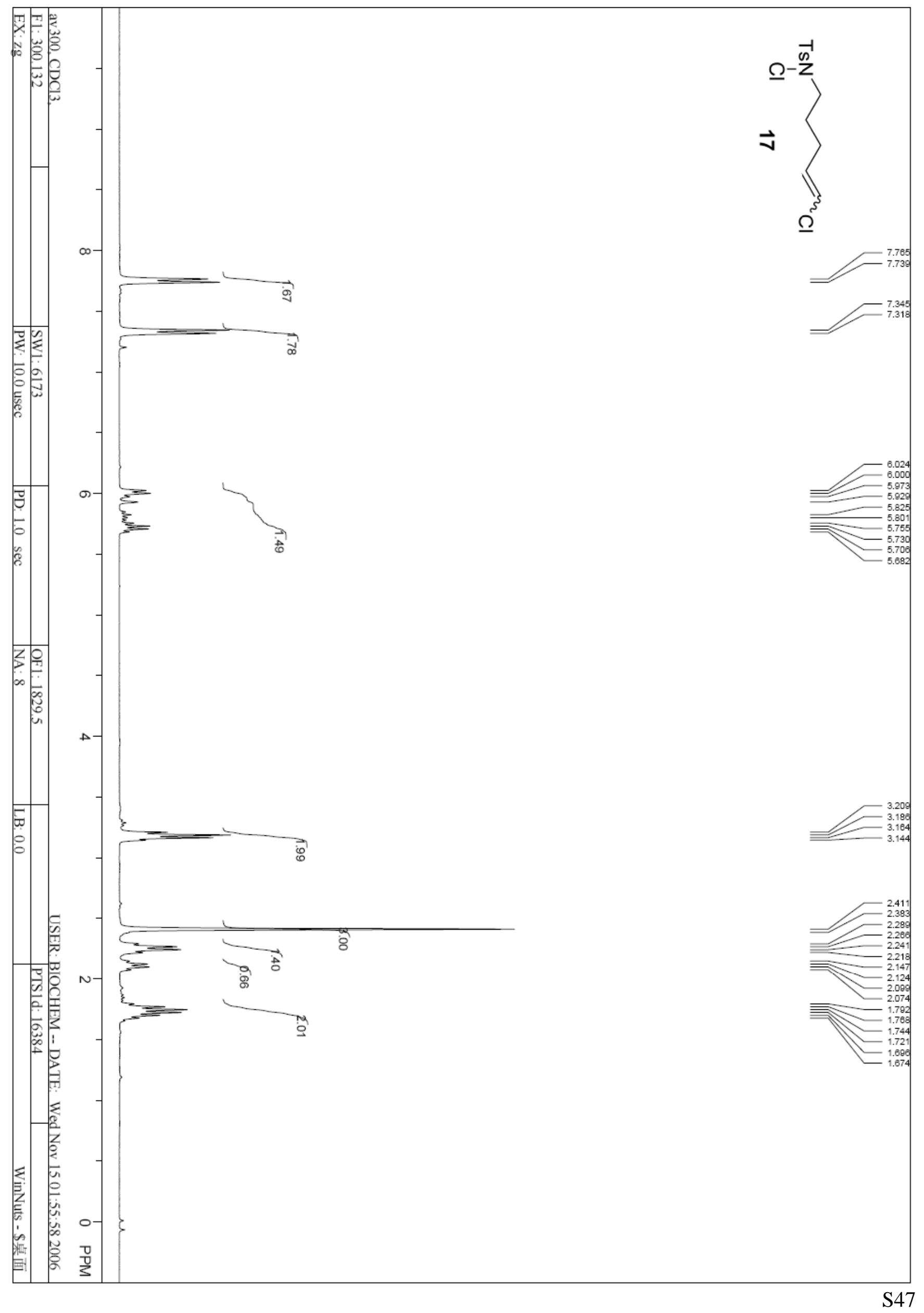




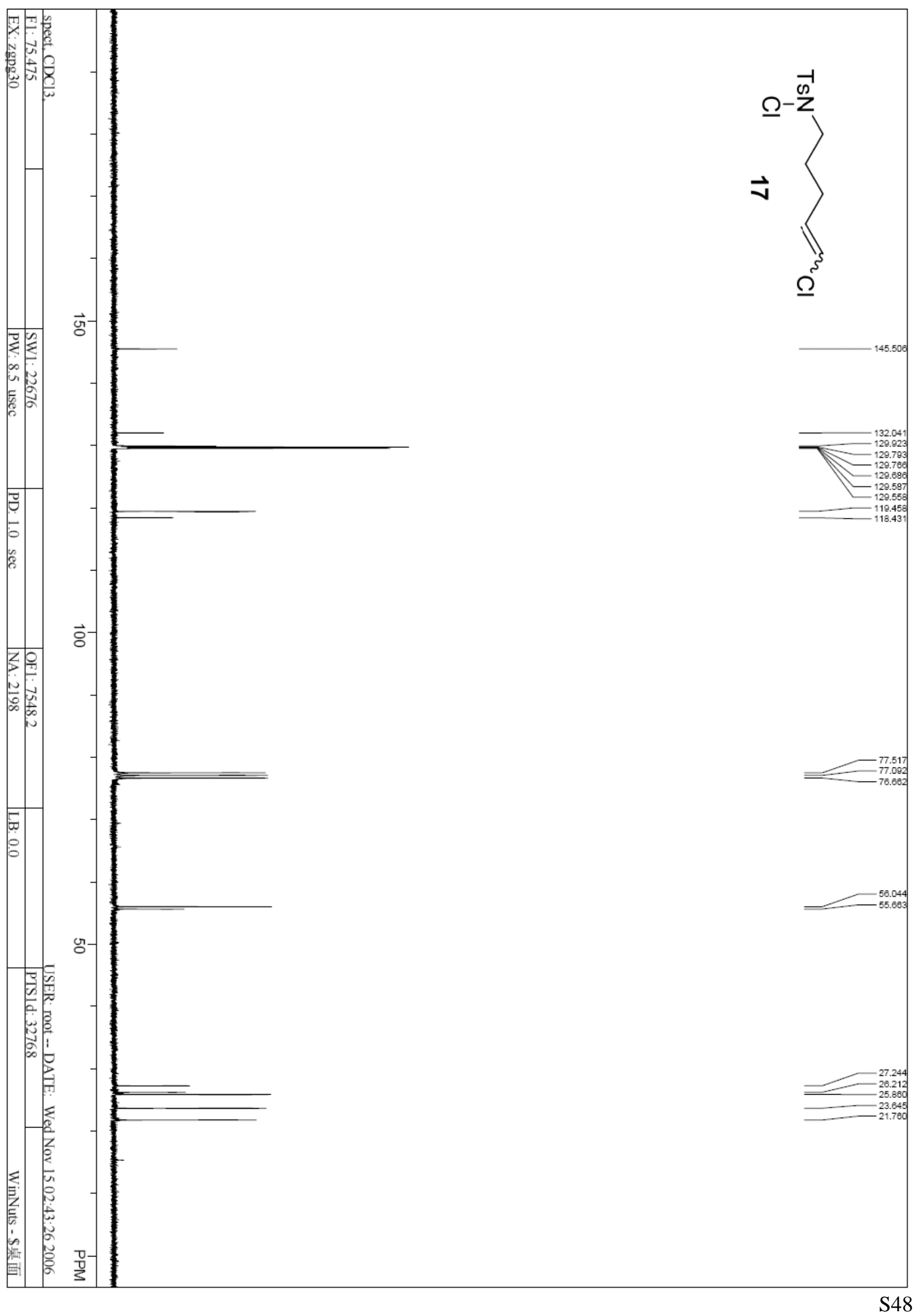




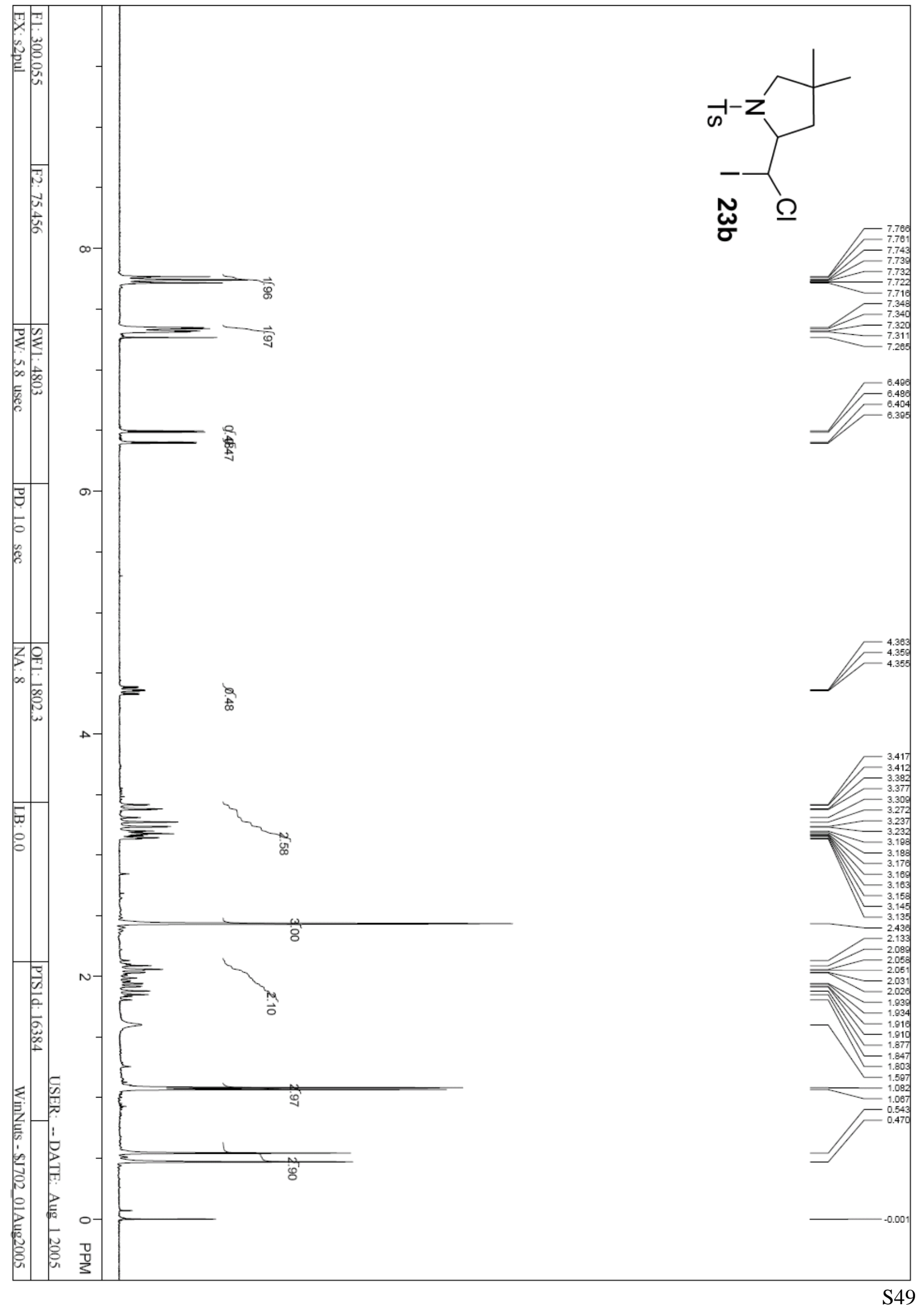




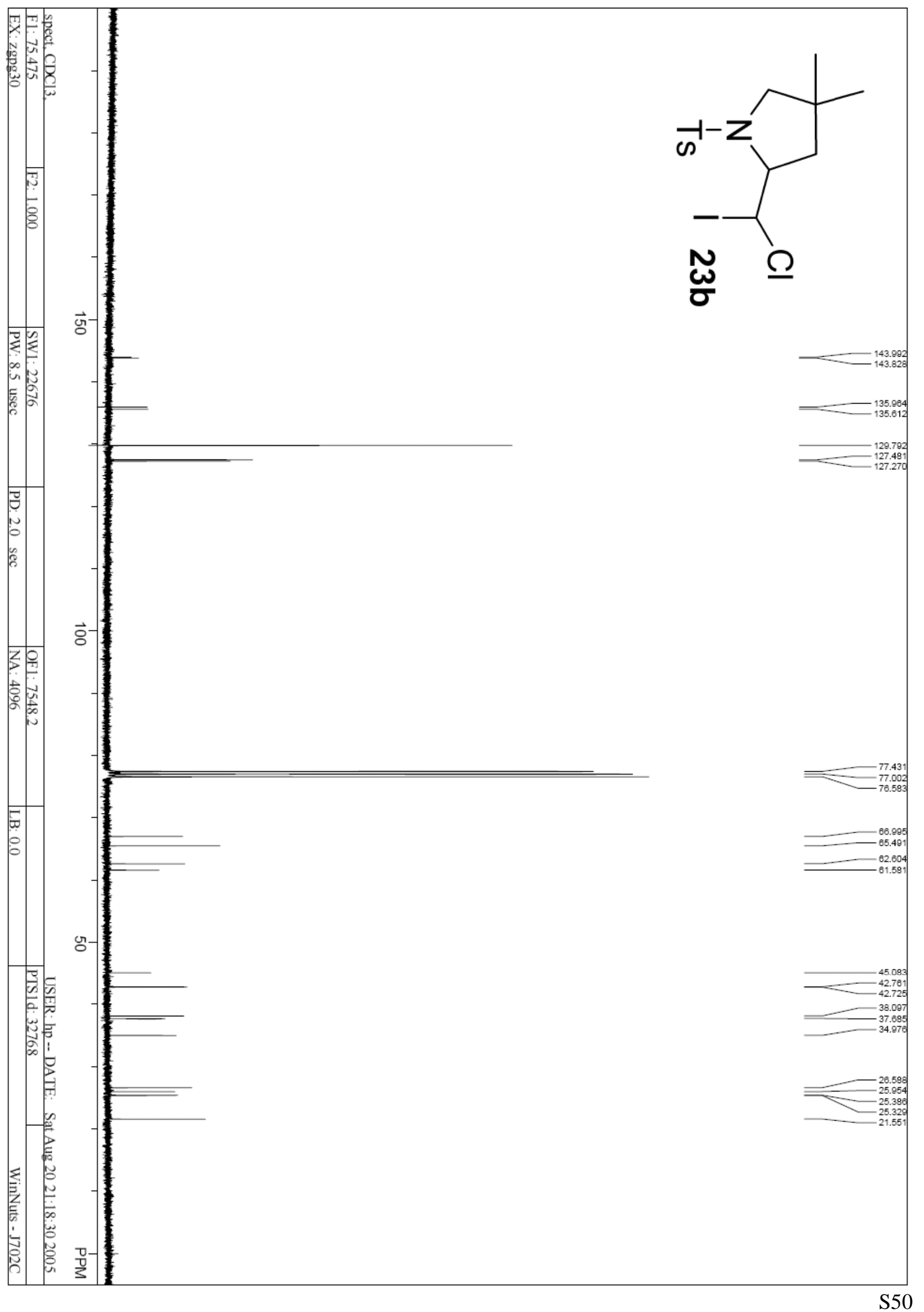




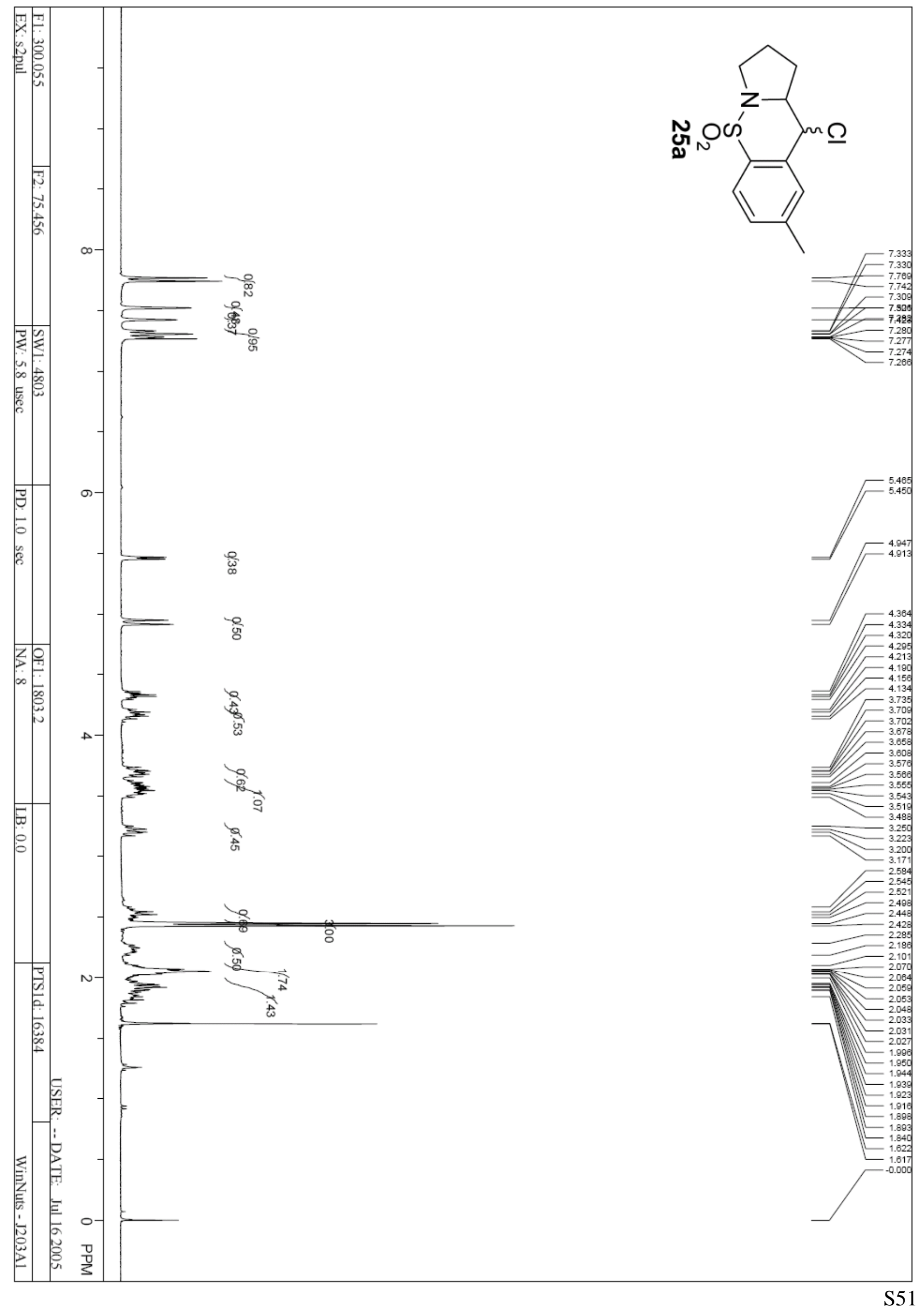




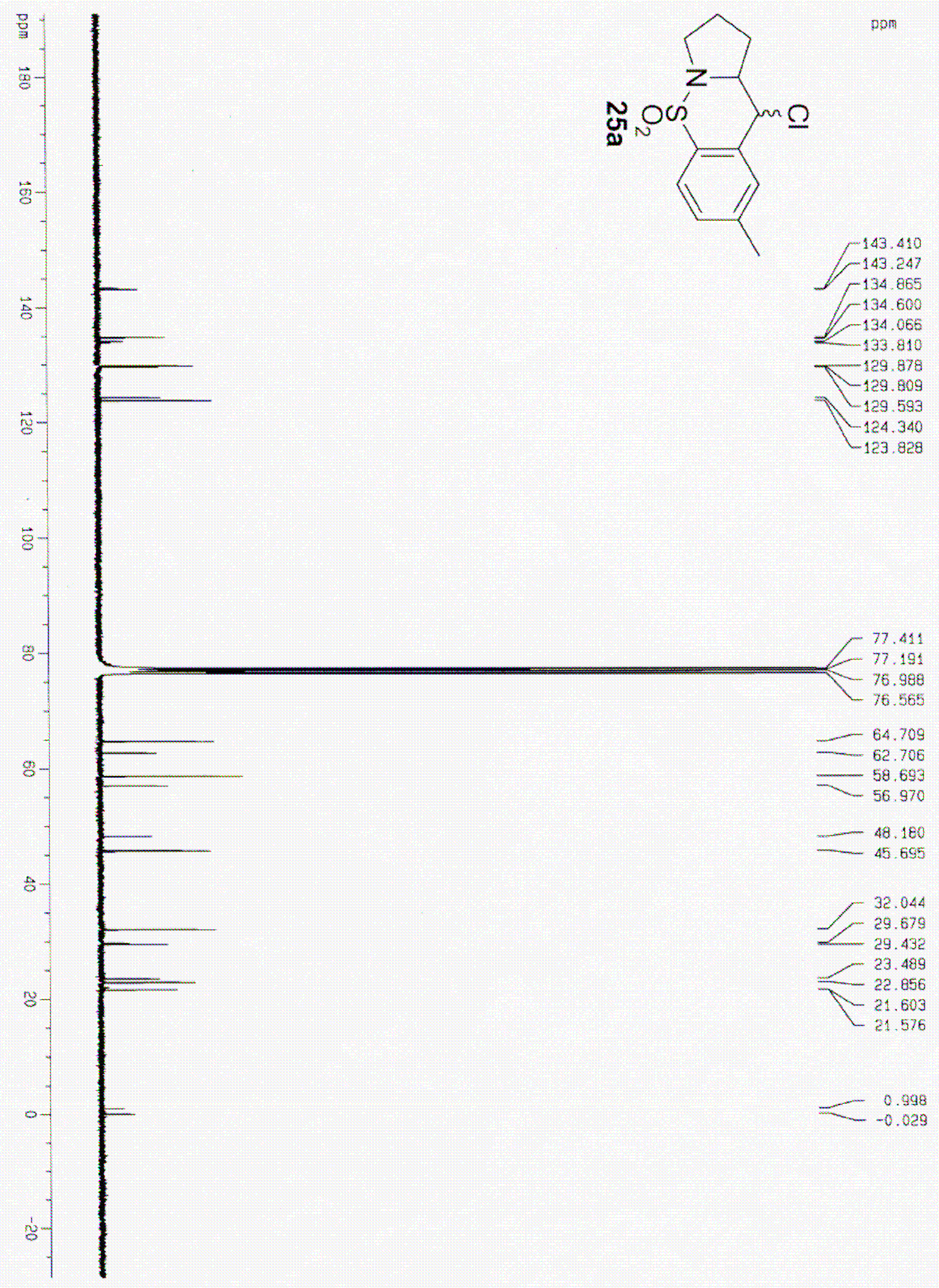




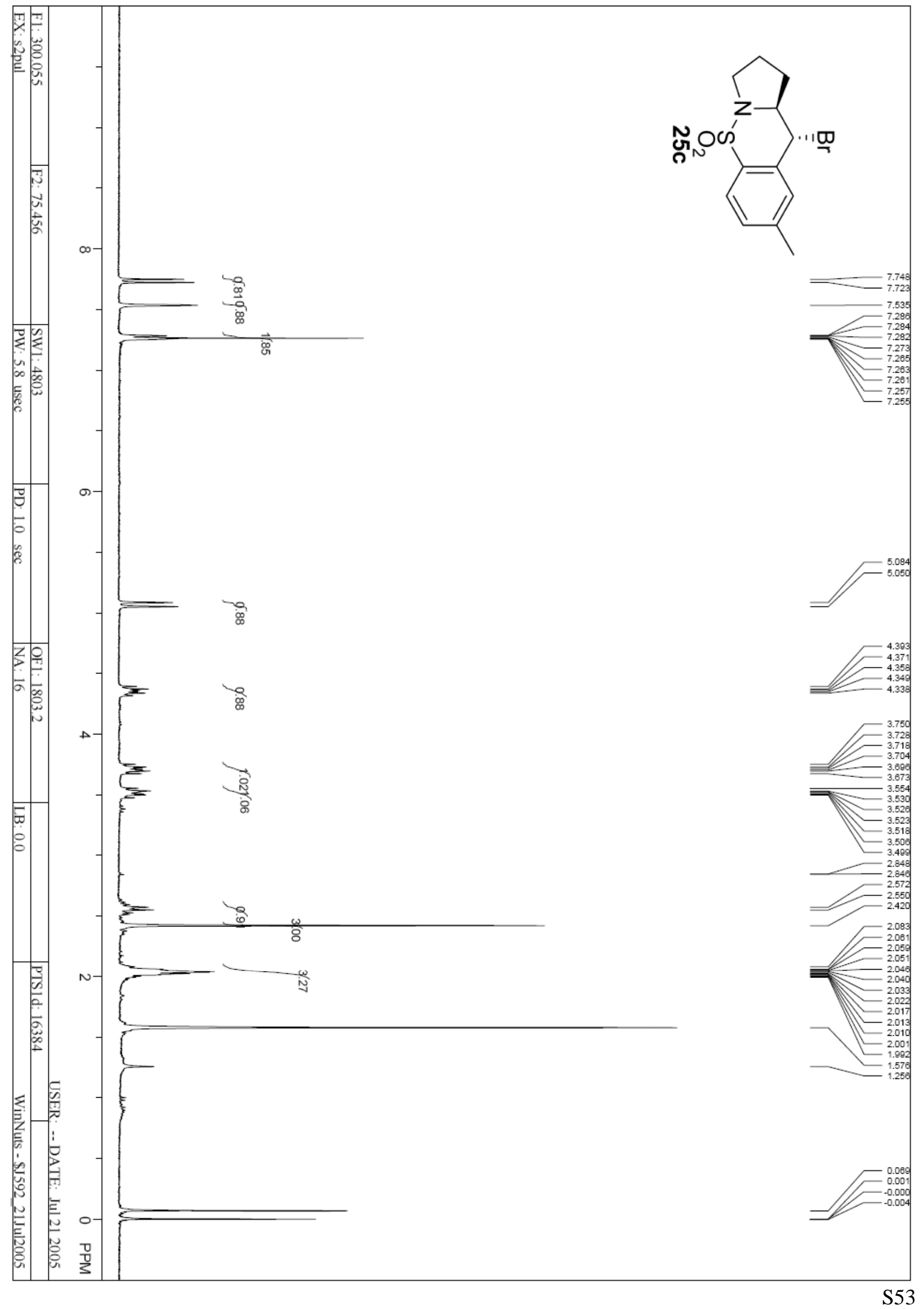









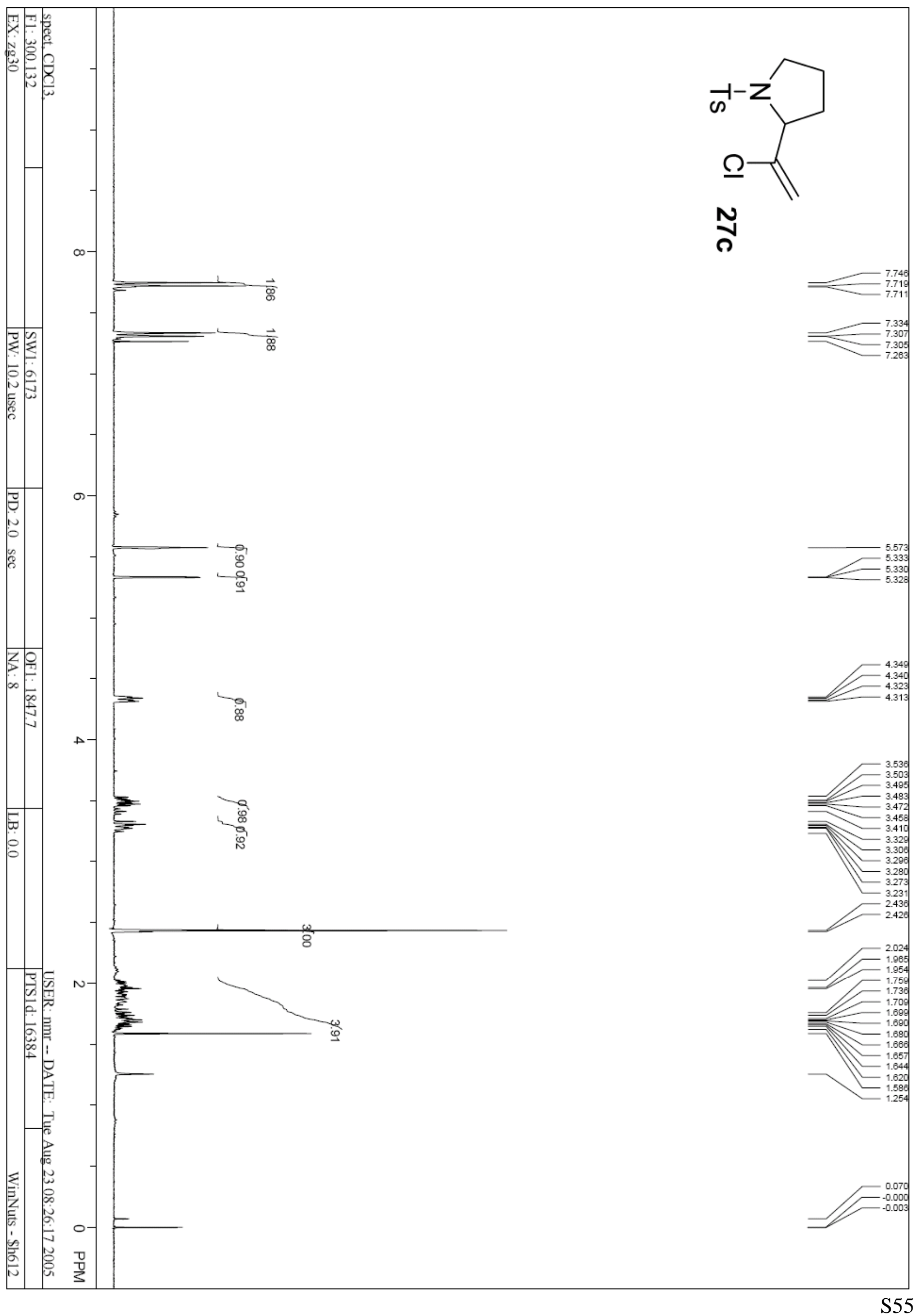



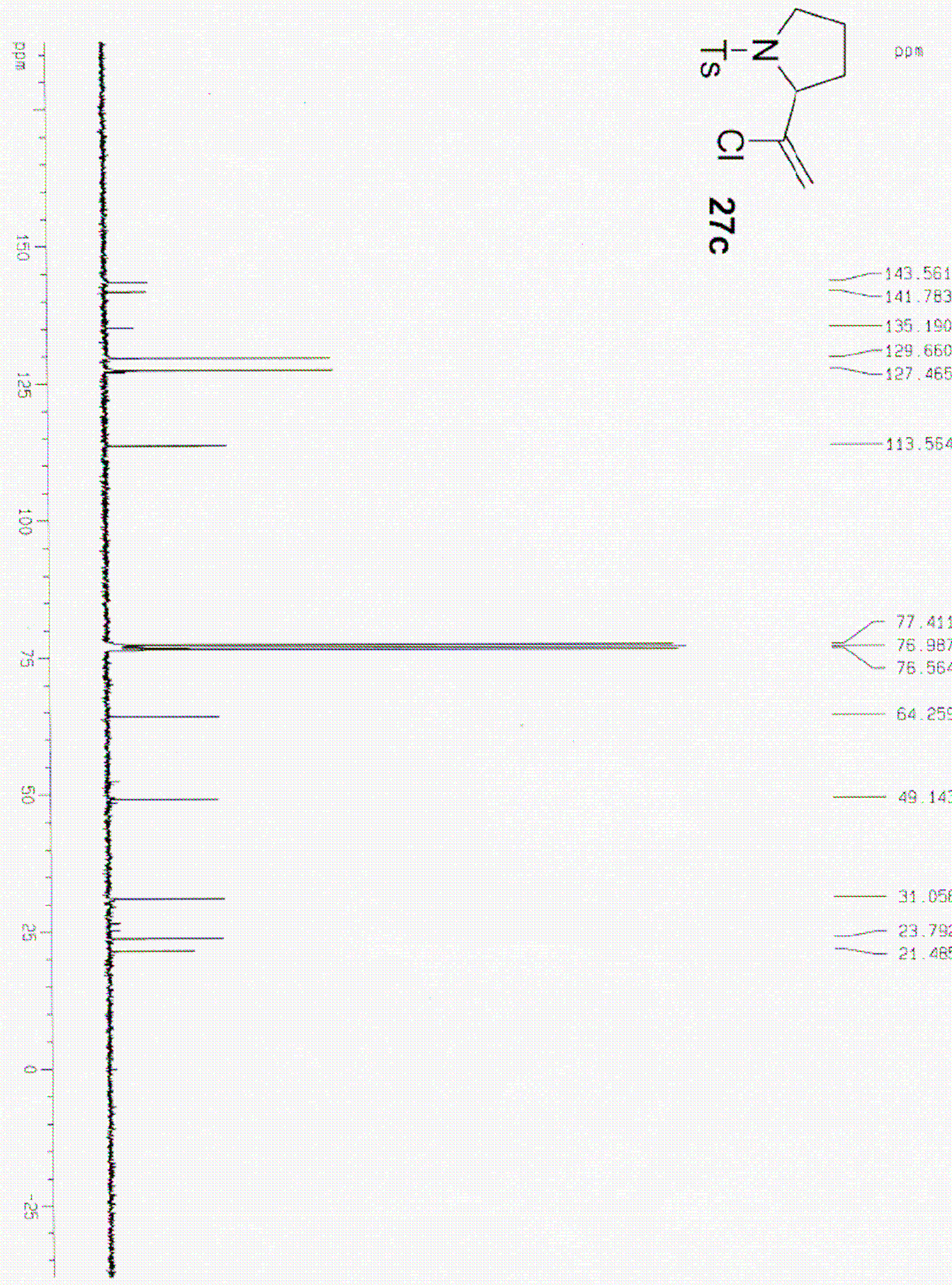

$-\quad 31.056$

$-\quad 23,792$

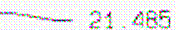




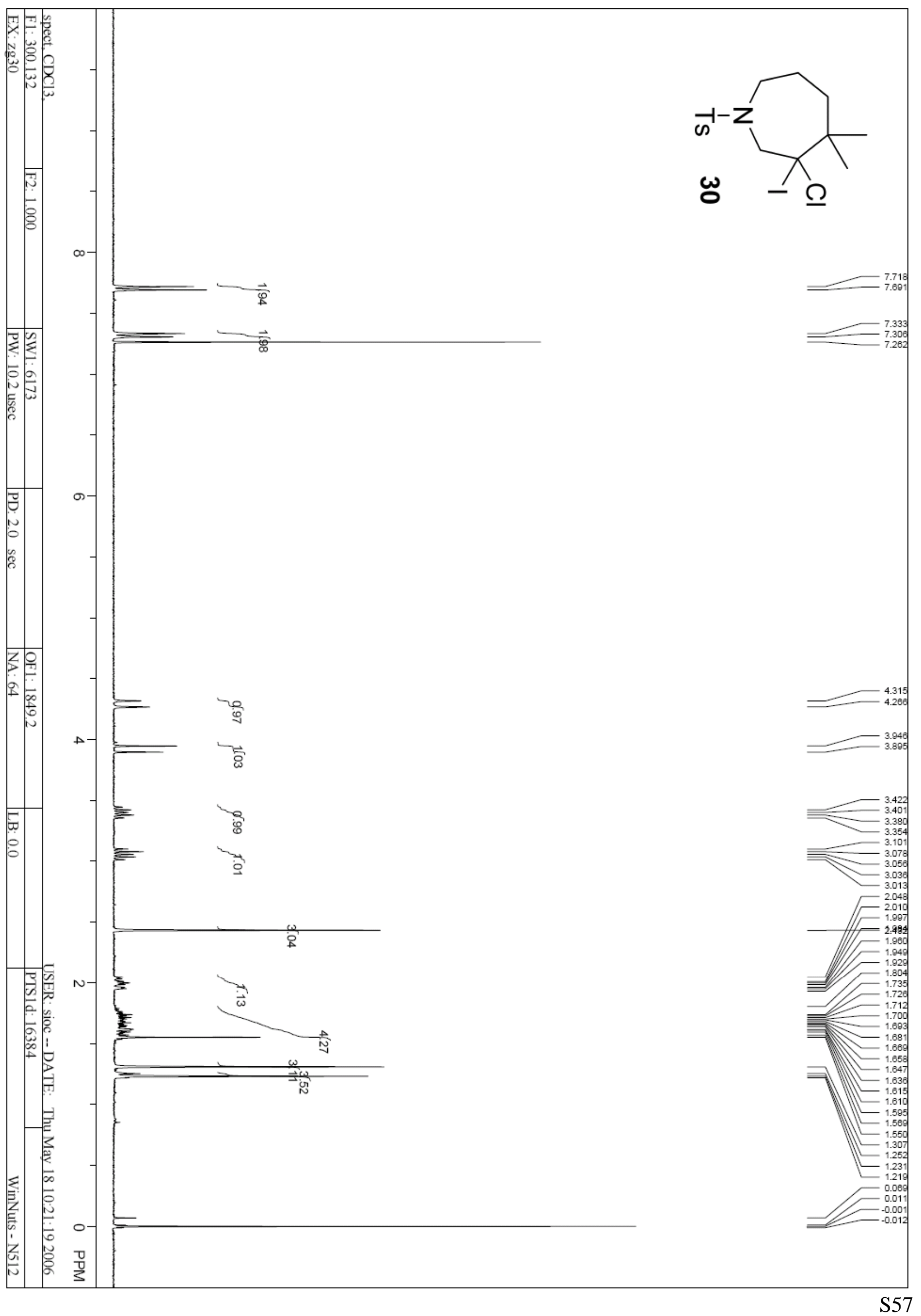




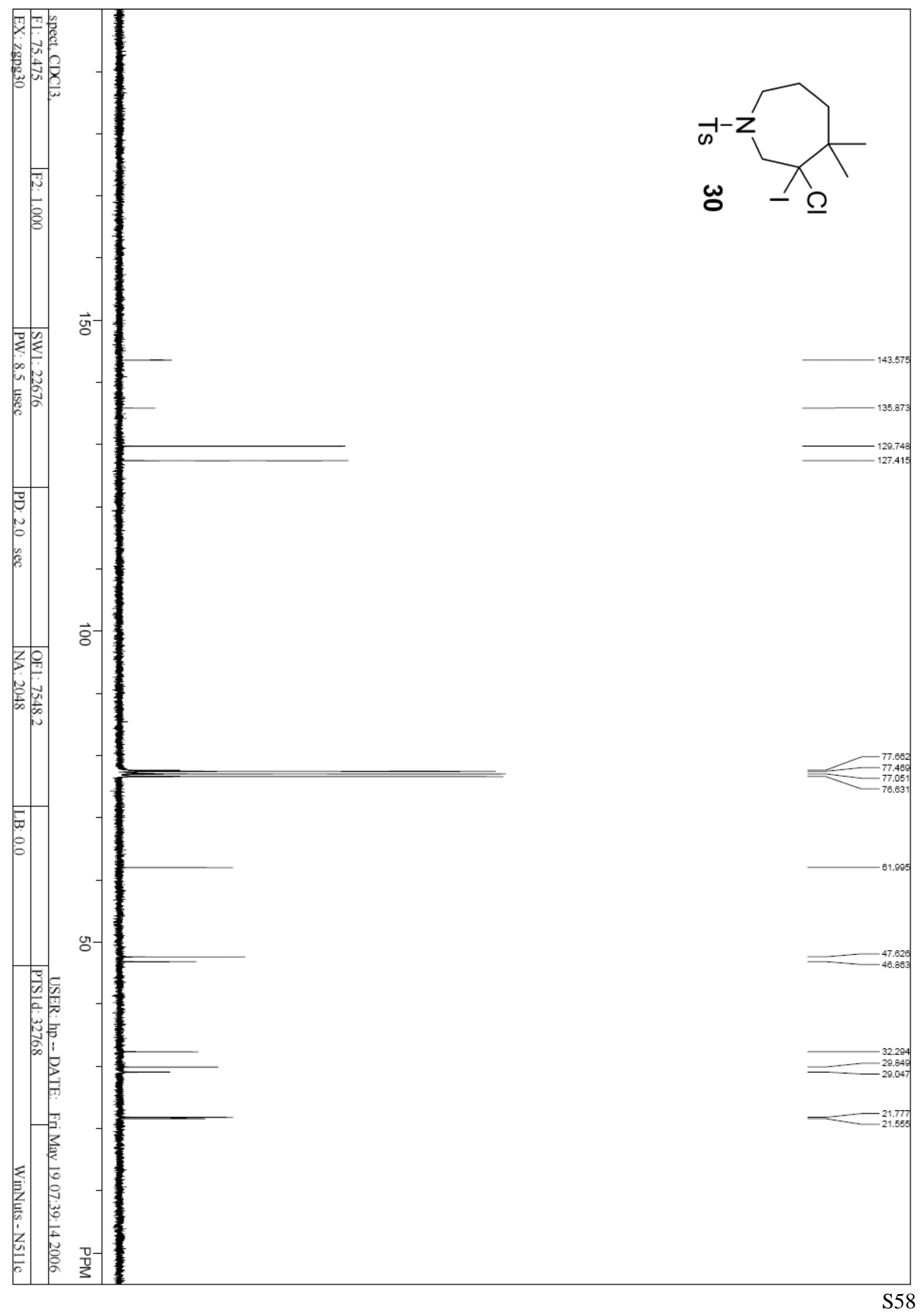




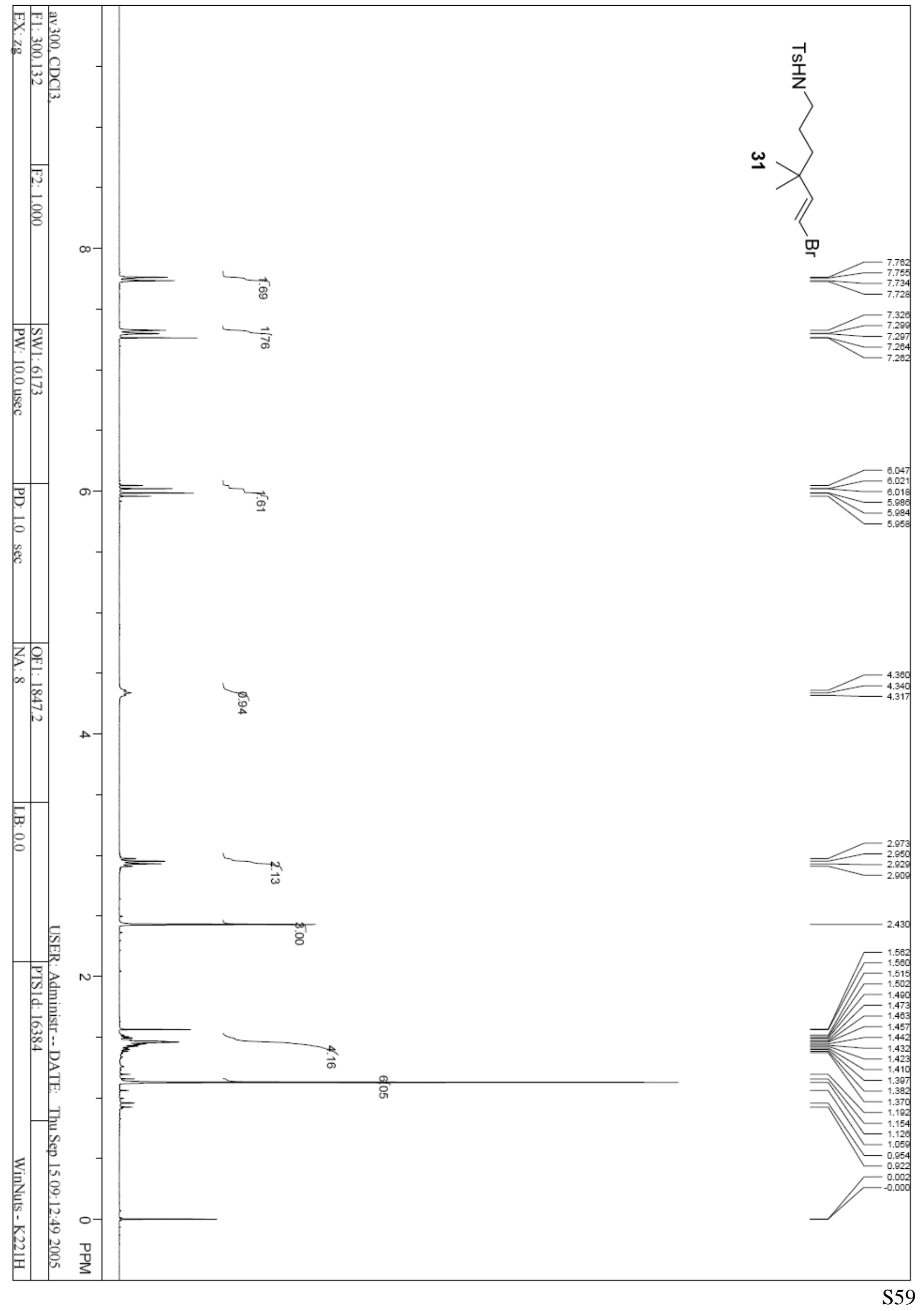




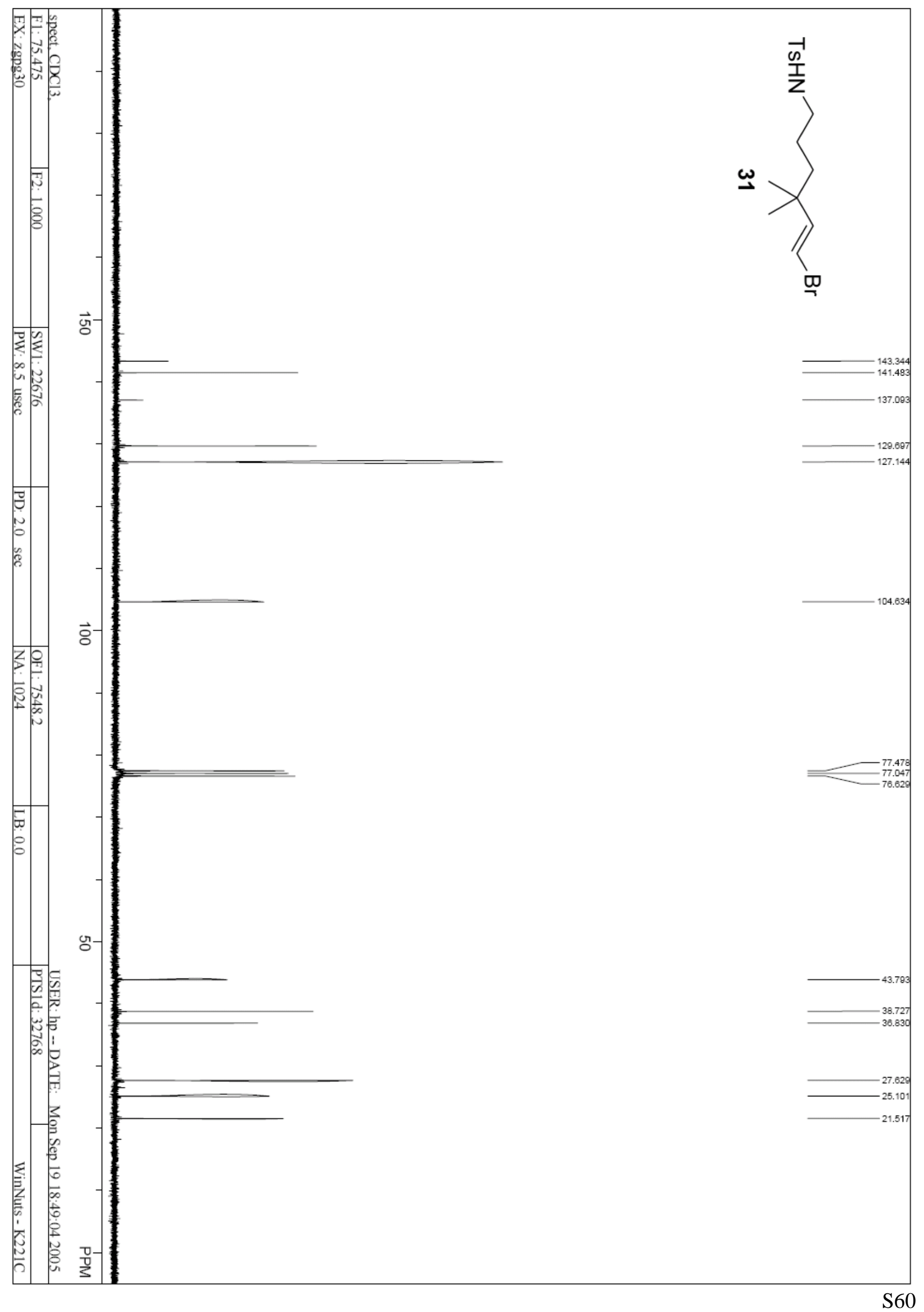

